\title{
SPECTRAL ANALYSIS OF THE LAPLACIAN ACTING ON DISCRETE CUSPS AND FUNNELS
}

\author{
NASSIM ATHMOUNI, MARWA ENNACEUR, AND SYLVAIN GOLÉNIA
}

\begin{abstract}
We study perturbations of the discrete Laplacian associated to discrete analogs of cusps and funnels. We perturb the metric and the potential in a long-range way. We establish a propagation estimate and a Limiting Absorption Principle away from the possible embedded eigenvalues. The approach is based on a positive commutator technique.
\end{abstract}

\section{Contents}

1. Introduction

2. The Mourre theory

3. The free model

3.1. Construction of the graph

3.2. Mourre estimate on $\mathbb{N}$

3.3. The funnel side

3.3.1. A first step into the analysis

3.3.2. Construction of the conjugate operator 10

3.4. The cusps side 11

3.4.1. The model and the low/high energy decomposition 12

3.4.2. The conjugate operator 13

3.5. The compact part 14

3.6. The whole graph 15

4. The perturbed model 15

4.1. Perturbation of the metric $\quad 15$

4.2. Main result $\quad 16$

4.3. The funnel side $\quad 17$

4.4. The cusp side: Radial metric perturbation 23

$\begin{array}{ll}\text { References } & 29\end{array}$

\section{INTRODUCTION}

The spectral theory of discrete Laplacians on graphs has drawn a lot of attention for decades as they are discrete analogs of manifolds. We are especially interested in the nature of the essential spectrum. Without trying to be exhaustive, using positive commutator techniques, [Sa, BoSa] treat the case of $\mathbb{Z}^{d}$, [AlFr, GeGo] study the case of binary trees, [MăRiTi] investigate some general graphs, and [PaRi] focused on a periodic setting. Some other techniques have been used successfully, e.g., [HiNo] with some geometric approach and [BrKe].

In the context of some manifolds of finite volume, [MoTr, GoMo] prove that the essential spectrum of the (continuous) Laplacian becomes empty under the presence of a magnetic field with compact support. Besides, they establish some

2010 Mathematics Subject Classification. 81Q10, 47B25, 47A10, 05C63.

Key words and phrases. commutator, Mourre estimate, limiting absorption principle, discrete Laplacian, locally finite graphs. 
Weyl asymptotic. Analogously, for some discrete cusps, [GoTr] classify magnetic potentials that lead to the absence of the essential spectrum and compute a kind of Weyl asymptotic for the magnetic discrete Laplacian. Back to [GoMo], one also obtains a refined analysis of the spectral measure (propagation estimate, limiting absorption principle) for long-range perturbation of the metric when the essential spectrum occurs relying on a positive commutator technique. We refer to [GoMo] for further comments and references therein. This part of the analysis was not carried out in [GoTr]. This is the main aim of this article.

To start off, we recall some standard definitions of graph theory. A (non-oriented) graph is a triple $\mathcal{G}:=(\mathcal{E}, \mathcal{V}, m)$, where $\mathcal{V}$ is a finite or countable set (the vertices), $\mathcal{E}: \mathcal{V} \times \mathcal{V} \rightarrow \mathbb{R}_{+}$is symmetric, and $m: \mathcal{V} \rightarrow(0, \infty)$ is a weight. We say that $\mathcal{G}$ is simple if $m=1$ and $\mathcal{E}: \mathcal{V} \times \mathcal{V} \rightarrow\{0,1\}$.

Given $x, y \in \mathcal{V}$, we say that $(x, y)$ is an edge and that $x$ and $y$ are neighbors if $\mathcal{E}(x, y)>0$. Note that in this case, since $\mathcal{E}$ is symmetric, $(y, x)$ is also an edge and $y$ and $x$ are neighbors. We denote this relationship by $x \sim y$ and the set of neighbors of $x$ by $\mathcal{N}_{\mathcal{G}}(x)$. The space of complex-valued functions acting on the set of vertices $\mathcal{V}$ is denoted by $C(\mathcal{V}):=\{f: \mathcal{V} \rightarrow \mathbb{C}\}$. Moreover, $C_{c}(\mathcal{V})$ is the subspace of $C(\mathcal{V})$ of functions with finite support. We consider the Hilbert space

$$
\ell^{2}(\mathcal{V}, m):=\left\{f \in C(\mathcal{V}), \quad \sum_{x \in \mathcal{V}} m(x)|f(x)|^{2}<\infty\right\}
$$

endowed with the scalar product, $\langle f, g\rangle:=\sum_{x \in \mathcal{V}} m(x) \overline{f(x)} g(x)$. We define the Laplacian operator

$$
\Delta_{\mathcal{G}} f(x):=\frac{1}{m(x)} \sum_{y \in \mathcal{V}} \mathcal{E}(x, y)(f(x)-f(y)),
$$

for all $f \in \mathcal{C}_{c}(\mathcal{V}) . \Delta_{\mathcal{G}}$ is a positive operator since we have $\left\langle f, \Delta_{\mathcal{G}} f\right\rangle_{\ell^{2}(\mathcal{V}, m)}=Q_{\mathcal{G}}(f)$, with

$$
Q_{\mathcal{G}}(f):=\frac{1}{2} \sum_{x, y \in \mathcal{V}} \mathcal{E}(x, y)|f(x)-f(y)|^{2},
$$

for all $f \in \mathcal{C}_{c}(\mathcal{V})$. To simplify, we denote its Friedrichs'extension with the same symbol. We define the degree of $x \in \mathcal{V}$ by

$$
\operatorname{deg}_{\mathcal{G}}(x):=\frac{1}{m(x)} \sum_{y \in \mathcal{V}} \mathcal{E}(x, y)
$$

We present a simple version of our model: We consider $\mathcal{G}_{1}:=\left(\mathcal{E}_{1}, \mathcal{V}_{1}, m_{1}\right)$, where $\mathcal{V}_{1}:=\mathbb{Z}, m_{1}(n):=e^{-n}$, and $\mathcal{E}(n, n+1):=e^{-(2 n+1) / 2}$, for all $n \in \mathbb{N}$ and $\mathcal{G}_{2}:=$ $\left(\mathcal{E}_{2}, \mathcal{V}_{2}, m_{2}\right)$ a connected finite graph such that $\left|\mathcal{V}_{2}\right|=p, p \geq 3$, where $\left|\mathcal{V}_{2}\right|$ is the cardinal of the set $\mathcal{V}_{2}$ with $m_{2}$ constant. Let $\mathcal{G}:=(\mathcal{E}, \mathcal{V}, m)$ be the twisted cartesian product $\mathcal{G}_{1} \times{ }_{\tau} \mathcal{G}_{2}$ given by

$$
\left\{\begin{aligned}
m(x, y):= & m_{1}(x) \times m_{2}(y) \\
\mathcal{E}\left((x, y),\left(x^{\prime}, y^{\prime}\right)\right):= & \mathcal{E}_{1}\left(x, x^{\prime}\right) \times \delta_{y, y^{\prime}}+\delta_{x, x^{\prime}} \times \mathcal{E}_{2}\left(y, y^{\prime}\right),
\end{aligned}\right.
$$

for all $x, x^{\prime} \in \mathcal{V}_{1}$ and $y, y^{\prime} \in \mathcal{V}_{2}$, If $n>0$, this is a cups side and if $n<0$, this is a funnel side. We refer to Section 3.1 for more details.

The (twisted cartesian) Laplacian $\Delta_{\mathcal{G}}$ is essentially self-adjoint on $\mathcal{C}_{c}(\mathcal{V})$, see Proposition 3.14. Moreover, it has no singularly continuous spectrum and

$$
\sigma_{\mathrm{ac}}\left(\Delta_{\mathcal{G}}\right)=\left[\frac{\alpha}{m_{2}}, \frac{\beta}{m_{2}}\right],
$$

with

$$
\alpha:=e^{1 / 2}+e^{-1 / 2}-2 \quad \text { and } \quad \beta:=e^{1 / 2}+e^{-1 / 2}+2 .
$$




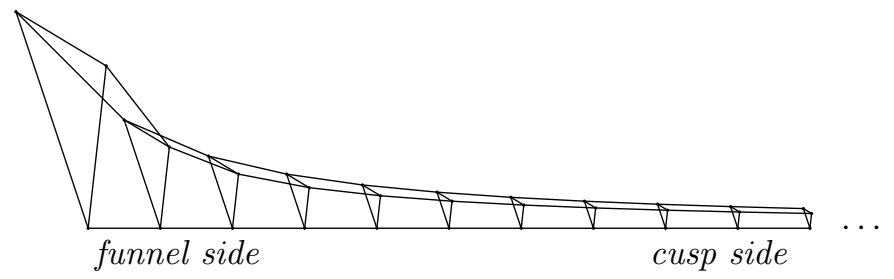

FiguRE 1. Representation of a discrete cusp and funnel side

We turn into perturbation theory. First, we perturb the weights, we consider $\mathcal{G}^{\prime}:=$ $\left(\mathcal{E}^{\prime}, \mathcal{V}, m^{\prime}\right)$, where

$$
m^{\prime}(x):=(1+\mu(x)) m(x) \text { and } \mathcal{E}^{\prime}(x, y):=(1+\varepsilon(x, y)) \mathcal{E}(x, y),
$$

$$
\begin{cases}\max _{x_{2} \in \mathcal{V}_{2}}\left|V\left(\left(x_{1}, x_{2}\right)\right)\right| \rightarrow 0, & \text { if }\left|x_{1}\right| \rightarrow \infty, \\ \max _{x_{2} \in \mathcal{V}_{2}}\left|\mu\left(\left(x_{1}, x_{2}\right)\right)\right| \rightarrow 0, & \text { if }\left|x_{1}\right| \rightarrow \infty, \\ \max _{x_{2} \in \mathcal{V}_{2}, y \sim\left(x_{1}, x_{2}\right)}\left|\varepsilon\left(\left(x_{1}, x_{2}\right), y\right)\right| \rightarrow 0, & \text { if }\left|x_{1}\right| \rightarrow \infty .\end{cases}
$$

This ensures that $\Delta_{\mathcal{G}^{\prime}}+V(\cdot)$ is also essentially self-adjoint on $\mathcal{C}_{c}(\mathcal{V})$. Here $V(\cdot)$ denotes the operator of multiplication by $V$. Moreover, $\left(H_{0}\right)$ guarantees the stability of the essential spectrum, see Proposition 4.2. Namely,

$$
\sigma_{\mathrm{ess}}\left(\Delta_{\mathcal{G}^{\prime}}\right)=\left[\frac{\alpha}{m_{2}}, \frac{\beta}{m_{2}}\right]
$$

In order to obtain the absence of singularly continuous spectrum for $\Delta_{\mathcal{G}^{\prime}}$, we require some additional decay. Let $\epsilon>0$ and ask:

$$
\begin{aligned}
& \left(H_{1}\right) \quad \sup _{n \in \mathbb{Z}, y \in \mathcal{V}_{2}}\langle n\rangle^{1+\epsilon}|V(n-1, y)-V(n, y)|<\infty, \\
& \left(H_{2}\right) \quad \sup _{n \in \mathbb{Z}, y \in \mathcal{V}_{2}}\langle n\rangle^{1+\epsilon}|\mu(n-1, y)-\mu(n, y)|<\infty \\
& \left(H_{3}\right) \quad \sup _{n \in \mathbb{Z}, k \in \mathcal{V}_{2}}\langle n\rangle^{1+\epsilon}|\varepsilon((n, k),(n+1, k))-\varepsilon((n-1, k),(n, k))|<\infty,
\end{aligned}
$$

where $\langle\cdot\rangle:=\sqrt{1+|\cdot|^{2}}$.

Our main result is the following:

Theorem 1.1. Let $H:=\Delta_{\mathcal{G}^{\prime}}+V(\cdot)$ as above. Suppose that $\left(H_{0}\right)$ holds true. Then, we have the following assertions:

(1) $\sigma_{\text {ess }}(H)=\sigma_{\text {ess }}\left(\Delta_{\mathcal{G}}\right)$.

Assume furthermore that $\left(H_{1}\right),\left(H_{2}\right)$, and $\left(H_{3}\right)$ hold true. Set $\kappa(H):=\sigma_{p}(H) \cup$ $\left\{\alpha / m_{2}, \beta / m_{2}\right\}$ with $\alpha, \beta$ are given in (1.2) and where $\sigma_{p}$ denotes the pure point spectrum. Take $s>1 / 2$ and $[a, b] \subset \mathbb{R} \backslash \kappa(H)$. We obtain:

(2) The eigenvalues of $H$ distinct from $\alpha / m_{2}$ and $\beta / m_{2}$ are of finite multiplicity and can accumulate only toward $\alpha / m_{2}$ and $\beta / m_{2}$.

(3) The singular continuous spectrum of $H$ is empty.

(4) The following limit exists and finite:

$$
\lim _{\rho \rightarrow 0} \sup _{\lambda \in[a, b]}\left\|\langle\Lambda\rangle^{-s}(H-\lambda-\mathrm{i} \rho)^{-1}\langle\Lambda\rangle^{-s}\right\|<\infty,
$$

(5) There exists $c>0$ such that for all $f \in \ell^{2}\left(\mathcal{V}, m^{\prime}\right)$, we have:

$$
\int_{\mathbb{R}}\left\|\langle\Lambda\rangle^{-s} e^{-\mathrm{i} t H} E_{[a, b]}(H) f\right\|_{\ell^{2}\left(\mathcal{V}, m^{\prime}\right)}^{2} d t \leq c\|f\|_{\ell^{2}\left(\mathcal{V}, m^{\prime}\right)}^{2} .
$$


Our approach is based on a positive commutator technique, namely we establish a Mourre estimate. The proof of this theorem is given in Subsection 4.2. We refer to Section 2 for historical references and for an introduction on the subject.

We now describe the structure of the paper. In Section 2, we present the Mourre's theory. The next section is devoted to study the free model. In Subsection 3.1, we present the context and introduce the notion of cusp and funnel. In Subsection 3.2 , we start with the Mourre estimate on $\mathbb{N}$. In Subsections 3.3, and 3.4, we prove the Mourre estimate for the unperturbed Laplacian that acts on a funnel and on a cusp, respectively. Then, in Subsection 3.6, we conclude the Mourre estimate for the whole graph. In Section 4, we perturb the metrics and add a potential. The proofs are more involved than in Section 3.1 as we rely on the optimal class $\mathcal{C}^{1,1}(\mathcal{A})$ of the Mourre theory. This yields the main result.

Notation: We denote by $\mathbb{N}$ the set of non-negative integers. In particular $0 \in \mathbb{N}$. Set $\llbracket a, b \rrbracket:=[a, b] \cap \mathbb{Z}$. We denote by $\mathbf{1}_{X}$ the indicator of the set $X$.

Acknowledgements: We would like to anonymous referees for their comments on the script.

\section{The Mourre Theory}

In $[\mathrm{Pu}]$, C.R. Putnam used a positive commutator estimate to insure that the spectrum of an operator is purely absolutely continuous. His method was unfortunately not very flexible and did not allow the presence of eigenvalue. In [Mo1, Mo2], E. Mourre had the idea to localise in energy the positive commutator estimate. Thanks to some hypothesis of regularity, he proved that the embedded eigenvalues can accumulated only at some thresholds, that the singularly continuous spectrum is empty and also established a limiting absorption principle, away from the eigenvalues and from the thresholds. Many papers have shown the power of Mourre's commutator theory for a wide class of self-adjoint operators, e.g., [BaFrSi, BoCaHäMi, CaGrHu, DeJa, FrHe, GeGéMø, GeGo, HuSi, JeMoPe, Sa]. We refer to [AmBoGe] for the optimised theory and to [GoJe1, GoJe2, Gé] for recent developments.

Let us now, briefly recall Mourre's commutator theory. The aim is to establish some spectral properties of a given (unbounded) self-adjoint operator $H$ acting in some complex and separable Hilbert space $\mathcal{H}$ with the help of an external unbounded and self-adjoint operator $\mathcal{A}$. Let $\|\cdot\|$ denote the norm of bounded operators on $\mathcal{H}$ and $\sigma(H)$ the spectrum of $H$. Recall that the latter is real. We endow $\mathcal{D}(H)$, the domain of $H$, with its graph norm We denote by $R(z):=(H-z)^{-1}$ the resolvent of $H$ in $z$. Take an other Hilbert space $\mathcal{K}$ such that there is a dense and injective embedding from $\mathcal{K}$ to $\mathcal{H}$, by identifying $\mathcal{H}$ with its antidual $\mathcal{H}^{*}$, we have: $\mathcal{K} \hookrightarrow \mathcal{H} \simeq \mathcal{H}^{*} \hookrightarrow \mathcal{K}^{*}$, with dense and injective embeddings.

We introduce some regularity classes with respect to $\mathcal{A}$ and follow [AmBoGe, Chapter 6]. Given $k \in \mathbb{N}$, we say that $H \in \mathcal{C}^{k}(\mathcal{A})$ if for all $f \in \mathcal{H}$, the map $\mathbb{R} \ni t \mapsto e^{\mathrm{it} \mathcal{A}}(H+\mathrm{i})^{-1} \mathrm{e}^{-\mathrm{it} \mathcal{A}_{\mathrm{f}}} \in \mathcal{H}$ has the usual $\mathcal{C}^{k}$ regularity. We say that $H \in \mathcal{C}^{k, u}(\mathcal{A})$ if the map $\mathbb{R} \ni t \mapsto e^{\text {it } \mathcal{A}}(H+\mathrm{i}) e^{-i t \mathcal{A}} \in \mathcal{B}(\mathcal{H})$ is of $\operatorname{class} \mathcal{C}^{k}(\mathbb{R}, \mathcal{B}(\mathcal{H}))$, where $\mathcal{B}(\mathcal{H})$ is endowed with the norm operator topology.

We start with an example, e.g., [GoJe1, Proposition 2.1].

Lemma 2.1. For $\phi, \varphi \in \mathcal{D}(A)$, the rank one operator $|\phi\rangle\langle\varphi|: \psi \mapsto\langle\varphi, \psi\rangle \phi$ is of class $\mathcal{C}^{1}(A)$ and

$$
[|\phi\rangle\langle\varphi|, A]=|\phi\rangle\langle A \varphi|-| A \phi\rangle\langle\varphi| \text {. }
$$

By induction, given $n \in \mathbb{N}$ and $\phi, \varphi \in \mathcal{D}\left(A^{n}\right),|\phi\rangle\langle\varphi|$ is of class $\mathcal{C}^{n}(A)$.

We turn to a criterion in term of commutator.

Theorem 2.2 ([AmBoGe, p.258]). Let $\mathcal{A}$ and $H$ be two self-adjoint operators in the Hilbert space $\mathcal{H}$. The following points are equivalent: 
(1) $H \in \mathcal{C}^{1}(\mathcal{A})$.

(2) For one (then for all) $z \notin \sigma(H)$, there is a finite $c$ such that

$$
|\langle\mathcal{A} f, R(z) f\rangle-\langle R(\bar{z}) f, \mathcal{A} f\rangle| \leq c\|f\|^{2}, \text { for all } f \in \mathcal{D}(\mathcal{A}) .
$$

(3) (a) There is a finite $c$ such that for all $f \in \mathcal{D}(\mathcal{A}) \cap \mathcal{D}(H)$ :

$$
|\langle\mathcal{A} f, H f\rangle-\langle H f, \mathcal{A} f\rangle| \leq c\left(\|H f\|^{2}+\|f\|^{2}\right) .
$$

(b) For some (then for all) $z \notin \sigma(H)$, the set

$$
\{f \in \mathcal{D}(\mathcal{A}), R(z) f \in \mathcal{D}(\mathcal{A}) \text { and } R(\bar{z}) f \in \mathcal{D}(\mathcal{A})\} \text { is a core for } \mathcal{A} \text {. }
$$

Note that (2) yields that the commutator $[\mathcal{A}, R(z)]$ extends to a bounded operator in the form sense. We shall denote the extension by $[\mathcal{A}, R(z)]_{\text {。 }}$. In the same way, from (3a), the commutator $[H, \mathcal{A}]$ extends to a unique element of $\mathcal{B}\left(\mathcal{D}(H), \mathcal{D}(H)^{*}\right)$ denoted by $[H, \mathcal{A}]_{\circ}$. Note that $\mathcal{D}(H)$ is endowed with the graph norm of $H$ and that $\mathcal{D}(H)^{*}$ denotes its anti-dual. Moreover, if $H \in \mathcal{C}^{1}(\mathcal{A})$ and $z \notin \sigma(H)$,

$$
\left[A,(H-z)^{-1}\right]_{0}=\underbrace{(H-z)^{-1}}_{\mathcal{H} \leftarrow \mathcal{D}(H)^{*}} \underbrace{[H, A]_{0}}_{\mathcal{D}(H)^{*} \leftarrow \mathcal{D}(H)} \underbrace{(H-z)^{-1}}_{\mathcal{D}(H) \leftarrow \mathcal{H}} .
$$

Here, we use the Riesz lemma to identify $\mathcal{H}$ with its anti-dual $\mathcal{H}^{*}$.

Note that, in practice, the condition (3.b) could be delicate to check. This is addressed by the next lemma.

Lemma 2.3 ([GoMo, Lemma A.2]). Let $\mathscr{D}$ be a subspace of $\mathcal{H}$ such that $\mathscr{D} \subset$ $\mathcal{D}(H) \cap \mathcal{D}(\mathcal{A}), \mathscr{D}$ is a core for $A$ and $H \mathscr{D} \subset \mathscr{D}$. Let $\left(\chi_{n}\right)_{n \in \mathbb{N}}$ be a family of bounded operators such that

(1) $\chi_{n} \mathscr{D} \subset \mathscr{D}, \chi_{n}$ tends strongly to 1 as $n \rightarrow \infty$, and $\sup _{n}\left\|\chi_{n}\right\|_{\mathcal{B}(\mathcal{D}(H))}<\infty$.

(2) $\mathcal{A} \chi_{n} f \rightarrow \mathcal{A} f$, for all $f \in \mathscr{D}$, as $n \rightarrow \infty$.

(3) There is $z \notin \sigma(H)$, such that $\chi_{n} R(z) \mathscr{D} \subset \mathscr{D}$ and $\chi_{n} R(\bar{z}) \mathscr{D} \subset \mathscr{D}$.

Suppose also that for all $f \in \mathscr{D}$

$$
\lim _{n \rightarrow \infty} \mathcal{A}\left[H, \chi_{n}\right] R(z) f=0 \text { and } \lim _{n \rightarrow \infty} \mathcal{A}\left[H, \chi_{n}\right] R(\bar{z}) f=0 .
$$

Finally, suppose that there is a finite $c$ such that

$$
|\langle\mathcal{A} f, H f\rangle-\langle H f, \mathcal{A} f\rangle| \leq c\left(\|H f\|^{2}+\|f\|^{2}\right), \text { for all } f \in \mathscr{D} .
$$

Then, one has $H \in \mathcal{C}^{1}(\mathcal{A})$.

We define other refined classes of regularity:

$$
\begin{aligned}
& \text { We say that } H \in \mathcal{C}^{0,1}(\mathcal{A}) \text { if } \int_{0}^{1}\left\|\left[(H+\mathrm{i})^{-1}, \mathrm{e}^{\mathrm{i} t \mathcal{A}}\right]\right\| \frac{d t}{t}<\infty \\
& \text { We say that } H \in \mathcal{C}^{1,1}(\mathcal{A}) \text { if } \int_{0}^{1}\left\|\left[\left[(H+\mathrm{i})^{-1}, \mathrm{e}^{\mathrm{i} t \mathcal{A}}\right], \mathrm{e}^{\mathrm{i} t \mathcal{A}}\right]\right\| \frac{d t}{t^{2}}<\infty
\end{aligned}
$$

Thanks to [AmBoGe, p. 205], it turns out that

Given an interval open interval $\mathcal{I}$, we denote by $E_{\mathcal{I}}(H)$ the spectral projection of $H$ above $\mathcal{I}$. We say that the Mourre estimate holds true for $H$ on $\mathcal{I}$ if there exist $c>0$ and a compact operator $K$ such that

$$
E_{\mathcal{I}}(H)[H, \mathrm{i} \mathcal{A}]_{\circ} E_{\mathcal{I}}(H) \geq E_{\mathcal{I}}(H)(c+K) E_{\mathcal{I}}(H),
$$

when the inequality is understood in the form sense. We say that we have a strict Mourre estimate holds for $H$ on the open interval $\mathcal{I}^{\prime}$ when there exists $c^{\prime}>0$ such that

$$
E_{\mathcal{I}^{\prime}}(H)[H, \mathrm{i} \mathcal{A}]_{\circ} \mathrm{E}_{\mathcal{I}^{\prime}}(\mathrm{H}) \geq \mathrm{c}^{\prime} \mathrm{E}_{\mathcal{I}^{\prime}}(\mathrm{H}) .
$$

Assuming $H \in \mathcal{C}^{1}(\mathcal{A}),(2.1)$, and $\lambda \in \mathcal{I}$ is not an eigenvalue, therefore there exists an open interval $\mathcal{I}^{\prime}$ that contains $\lambda$ and $c^{\prime}>0$ such that (2.2). The aim of Mourre's 
commutator theory is to show a limiting absorption principle (LAP), see [AmBoGe, Theorem 7.6.8].

Theorem 2.4. Let $H$ be a self-adjoint operator, with $\sigma(H) \neq \mathbb{R}$. Assume that $H \in \mathcal{C}^{1}(\mathcal{A})$ and the Mourre estimate (2.1) holds true for $H$ on $\mathcal{I}$. Then

(1) The number of eigenvalues (counted with multiplicity) of $H$, that are in $\mathcal{I}$, is finite.

Assuming furthermore that $K=0$ in (2.1), it yields:

(2) $H$ has no eigenvalues in $\mathcal{I}$.

(3) If $H \in \mathcal{C}^{1,1}(\mathcal{A})$ and $K=0, s>1 / 2$ and $\mathcal{I}^{\prime}$ a compact sub-interval of $\mathcal{I}$, then

$$
\sup _{\Re(z) \in \mathcal{I}^{\prime}, \Im(z) \neq 0}\left\|\langle\mathcal{A}\rangle^{-s}(H-z)^{-1}\langle\mathcal{A}\rangle^{-s}\right\| \text { exists and finite. }
$$

Moreover, in the norm topology of bounded operators, the boundary values of the resolvent:

$$
\mathcal{I}^{\prime} \ni \lambda \mapsto \lim _{\rho \rightarrow 0^{ \pm}}\langle\mathcal{A}\rangle^{-s}(H-\lambda-\mathrm{i} \rho)^{-1}\langle\mathcal{A}\rangle^{-s} \text { exists and continuous. }
$$

For more details and deeper results, see [AmBoGe, Proposition 7.2.10, Corollary 7.2.11, Theorem 7.5.2].

\section{The FREe MODEL}

3.1. Construction of the graph. We discuss two different product of graphs. To start off, given $\mathcal{G}_{1}:=\left(\mathcal{E}_{1}, \mathcal{V}_{1}, m_{1}\right)$ and $\mathcal{G}_{2}:=\left(\mathcal{E}_{2}, \mathcal{V}_{2}, m_{2}\right)$, the Cartesian product of $\mathcal{G}_{1}$ by $\mathcal{G}_{2}$ is defined by $\mathcal{G}^{\diamond}:=\left(\mathcal{E}^{\diamond}, \mathcal{V}^{\diamond}, m^{\diamond}\right)$, where $\mathcal{V}^{\diamond}:=\mathcal{V}_{1} \times \mathcal{V}_{2}$,

$$
\left\{\begin{aligned}
m^{\diamond}(x, y) & :=m_{1}(x) \times m_{2}(y), \\
\mathcal{E}^{\diamond}\left((x, y),\left(x^{\prime}, y^{\prime}\right)\right) & :=\mathcal{E}_{1}\left(x, x^{\prime}\right) \times \delta_{y, y^{\prime}} m_{2}(y)+m_{1}(x) \delta_{x, x^{\prime}} \times \mathcal{E}_{2}\left(y, y^{\prime}\right) .
\end{aligned}\right.
$$

We denote it by $\mathcal{G}_{1} \times \mathcal{G}_{2}:=\mathcal{G}^{\diamond}$. This definition generalises the unweighted Cartesian product, e.g., [Ha]. It is used in several places in the literature, e.g., see [Ch, Section 2.6] and see [BoKeGoLiMü] for a generalisation.

The terminology is motivated by the following decomposition:

$$
\Delta_{\mathcal{G}^{\diamond}}=\Delta_{\mathcal{G}_{1}} \otimes 1+1 \otimes \Delta_{\mathcal{G}_{2}}
$$

where $\ell^{2}(\mathcal{V}, m) \simeq \ell^{2}\left(\mathcal{V}_{1}, m_{1}\right) \otimes \ell^{2}\left(\mathcal{V}_{2}, m_{2}\right)$. Note that

$$
e^{\mathrm{i} t \Delta_{\mathcal{G}^{\diamond}}}=e^{\mathrm{i} t \Delta_{\mathcal{G}_{1}}} \otimes e^{\mathrm{i} t \Delta_{\mathcal{G}_{2}}}, \quad \forall t \in \mathbb{R} .
$$

We refer to [ReSi, Section VIII.10] for an introduction to the tensor product of self-adjoint operators.

We now introduce a twisted Cartesian product. We refer to [GoTr, Section 2.2] for motivations, its link with hyperbolic geometry and generalisations. Given $\mathcal{G}_{1}:=$ $\left(\mathcal{E}_{1}, \mathcal{V}_{1}, m_{1}\right)$ and $\mathcal{G}_{2}:=\left(\mathcal{E}_{2}, \mathcal{V}_{2}, m_{2}\right)$, we define the product of $\mathcal{G}_{1}$ by $\mathcal{G}_{2}$ by $\mathcal{G}:=$ $(\mathcal{E}, \mathcal{V}, m)$, where $\mathcal{V}:=\mathcal{V}_{1} \times \mathcal{V}_{2}$ and

$$
\left\{\begin{aligned}
m(x, y) & :=m_{1}(x) \times m_{2}(y), \\
\mathcal{E}\left((x, y),\left(x^{\prime}, y^{\prime}\right)\right) & :=\mathcal{E}_{1}\left(x, x^{\prime}\right) \times \delta_{y, y^{\prime}}+\delta_{x, x^{\prime}} \times \mathcal{E}_{2}\left(y, y^{\prime}\right),
\end{aligned}\right.
$$

for all $x, x^{\prime} \in \mathcal{V}_{1}$ and $y, y^{\prime} \in \mathcal{V}_{2}$. We denote $\mathcal{G}$ by $\mathcal{G}_{1} \times_{\tau} \mathcal{G}_{2}$. If $m=1$, note that $\mathcal{G}_{1} \times{ }_{\tau} \mathcal{G}_{2}=\mathcal{G}_{1} \times \mathcal{G}_{2}$.

Under the representation $\ell^{2}(\mathcal{V}, m) \simeq \ell^{2}\left(\mathcal{V}_{1}, m_{1}\right) \otimes \ell^{2}\left(\mathcal{V}_{2}, m_{2}\right)$,

$$
\operatorname{deg}_{\mathcal{G}_{1} \times{ }_{\tau} \mathcal{G}_{2}}(\cdot)=\operatorname{deg}_{\mathcal{G}_{1}}(\cdot) \otimes \frac{1}{m_{2}(\cdot)}+\frac{1}{m_{1}(\cdot)} \otimes \operatorname{deg}_{\mathcal{G}_{2}}(\cdot)
$$

and

$$
\Delta_{\mathcal{G}_{1} \times{ }_{\tau} \mathcal{G}_{2}}=\Delta_{\mathcal{G}_{1}} \otimes \frac{1}{m_{2}(\cdot)}+\frac{1}{m_{1}(\cdot)} \otimes \Delta_{\mathcal{G}_{2}}
$$


If $m$ is non-trivial, we stress that the Laplacian obtained with our product is usually not unitarily equivalent to the Laplacian obtained with the Cartesian product.

A hyperbolic manifold of finite volume is the union of a compact part, of a cusp, and a funnel, e.g., [Th, Theorem 4.5.7]. In this article we study a discrete analog.

\section{In the sequel, we take $m_{2}$ constant on $\mathcal{V}_{2}$.}

The graph $\mathcal{G}:=(\mathcal{E}, \mathcal{V}, m)$ is divided into three parts: A cusp part, a funnel part, and a finite part. Set $\mathcal{G}^{\star}:=\left(\mathcal{E}^{\star}, \mathcal{V}^{\star}, m^{\star}\right)$ be the induced graph of $\mathcal{G}$ over $\mathcal{V}^{\star}$ where $\star \in\{\mathrm{c}, \mathrm{f}, 0\}$ and $\mathcal{V}$ is a disjoint reunion of $\mathcal{V}^{\mathrm{c}}, \mathcal{V}^{\mathrm{f}}$, and $\mathcal{V}^{0}$.

We consider $\mathcal{G}_{1}^{\mathrm{c}}:=\left(\mathcal{E}_{1}^{\mathrm{c}}, \mathcal{V}_{1}^{\mathrm{c}}, m_{1}^{\mathrm{c}}\right)$, where

$$
\mathcal{V}_{1}^{\mathrm{c}}:=\mathbb{N}, \quad m_{1}^{\mathrm{c}}(n):=\exp (-n), \text { and } \mathcal{E}_{1}^{\mathrm{c}}(n, n+1):=\exp (-(2 n+1) / 2),
$$

for all $n \in \mathbb{N}$ and $\mathcal{G}_{2}^{\mathrm{c}}:=\left(\mathcal{E}_{2}^{\mathrm{c}}, \mathcal{V}_{2}^{\mathrm{c}}, m_{2}\right)$ a possibly disconnected connected finite graph. Set $\mathcal{G}^{\mathrm{c}}:=\mathcal{G}_{1}^{\mathrm{c}} \times{ }_{\tau} \mathcal{G}_{2}^{\mathrm{c}}$. This is a cusp part. Note it is of finite volume as:

$$
\sum_{(x, y) \in \mathcal{V}_{1}^{c} \times \mathcal{V}_{2}^{c}} m_{\mathcal{G}^{c}}(x, y)<\infty .
$$

We consider $\mathcal{G}_{1}^{\mathrm{f}}:=\left(\mathcal{E}_{1}^{\mathrm{f}}, \mathcal{V}_{1}^{\mathrm{f}}, m_{1}^{\mathrm{f}}\right)$, where

$$
\mathcal{V}_{1}^{\mathrm{f}}:=\mathbb{N}, \quad m_{1}^{\mathrm{f}}(n):=\exp (n), \text { and } \mathcal{E}_{1}^{\mathrm{f}}(n, n+1):=\exp ((2 n+1) / 2),
$$

for all $n \in \mathbb{N}$ and $\mathcal{G}_{2}^{\mathrm{f}}:=\left(\mathcal{E}_{2}^{\mathrm{f}}, \mathcal{V}_{2}^{\mathrm{f}}, m_{2}\right)$ a connected finite graph. Set $\mathcal{G}^{\mathrm{f}}:=\mathcal{G}_{1}^{\mathrm{f}} \times_{\tau} \mathcal{G}_{2}^{\mathrm{f}}$. This is a funnel part.

For the compact part, we ask that for all $x \in \mathcal{V}^{0}, \operatorname{supp}(\mathcal{E}(x, \cdot))$ is finite and $m^{0}(x)>0$.

We now, take advantage of

$$
\ell^{2}(\mathcal{G}):=\ell^{2}\left(\mathcal{G}^{\mathrm{f}}\right) \oplus \ell^{2}\left(\mathcal{G}^{0}\right) \oplus \ell^{2}\left(\mathcal{G}^{\mathrm{c}}\right)
$$

We have that

$$
\Delta_{\mathcal{G}}:=\Delta_{\mathcal{G}^{\mathrm{f}}} \oplus 0 \oplus \Delta_{\mathcal{G}^{\mathrm{c}}}+\mathrm{K}_{0},
$$

where $K_{0}$ is an operator of finite rank with support in $\mathcal{C}^{c}(\mathcal{V})$.

To analyse the perturbations of operator we shall rely on the following gauge transformation, e.g., [Go, CoToTr, HaKe]. See also [BoGo] for some historical references.

Proposition 3.1. Let $\mathcal{G}:=(\mathcal{V}, \mathcal{E}, m)$ be a weighted graph and $m: \mathcal{V} \rightarrow(0, \infty)$ be a weight. The following map is unitary:

$$
\begin{aligned}
T_{m \rightarrow m^{\prime}} f: \ell^{2}(\mathcal{V}, m) & \rightarrow \ell^{2}\left(\mathcal{V}, m^{\prime}\right) \\
f & \mapsto\left(x \mapsto \sqrt{\frac{m(x)}{m^{\prime}(x)}} f(x)\right) .
\end{aligned}
$$

We have:

$$
\Delta_{\mathcal{G}^{\prime}}^{\mathcal{F}}=T_{m \rightarrow m^{\prime}}\left(\Delta_{\widetilde{\mathcal{G}}}-W(\cdot)\right)^{\mathcal{F}} T_{m \rightarrow m^{\prime}}^{-1},
$$

where $\mathcal{G}^{\prime}:=\left(\mathcal{V}, \mathcal{E}^{\prime}, m^{\prime}\right), \widetilde{\mathcal{G}}:=(\mathcal{V}, \widetilde{\mathcal{E}}, m)$ and,

$$
\begin{aligned}
\widetilde{\mathcal{E}}(x, y) & :=\mathcal{E}^{\prime}(x, y) \sqrt{\frac{m(x) m(y)}{m^{\prime}(x) m^{\prime}(y)}} \\
W(x) & :=\frac{1}{m(x)} \sum_{y \in \mathcal{V}} \widetilde{\mathcal{E}}(x, y)\left(1-\sqrt{\frac{m(x) m^{\prime}(y)}{m(y) m^{\prime}(x)}}\right) .
\end{aligned}
$$

Here we emphasised the choice Friedrichs extension with the symbol $\mathcal{F}$. 
3.2. Mourre estimate on $\mathbb{N}$. In this section we make a preliminary work on the half axis. We construct a conjugate operator, prove a Mourre estimate for $\Delta_{\mathbb{N}}$ and check the regularity conditions. This is a known result, e.g., [AlFr], see also [GeGo, Mic].

Given $f \in \ell^{2}(\mathbb{N}, 1)$, we set

$$
\forall n \in \mathbb{N}^{*}, \quad U f(n):=f(n-1) \text { and } U f(0):=0 .
$$

Note that $U^{*} f(n)=f(n+1), \forall n \in \mathbb{N}$. The operator $U$ is an isometry and is not unitary: we have $U^{*} U=$ id and $U U^{*}=\mathbf{1}_{[1, \infty[}(\cdot)$.

We define by $Q$ the operator of multiplication by $n$ in $\ell^{2}(\mathbb{N}, 1)$. Namely, it is the closure of the operator given by $(Q f)(n)=n f(n)$ for all $n \in \mathbb{N}$ and $f \in \mathcal{C}_{c}(\mathbb{N})$. It is essentially self-adjoint on $\mathcal{C}_{c}(\mathbb{N})$. In [GeGo], one finds the following elementary relations:

$$
Q U=U(Q+1), U^{*} Q=(Q+1) U^{*} \text { and } U Q U=U^{2}(Q+1) \quad \text { on } \mathcal{D}(Q) .
$$

The operator $\Delta_{\mathbb{N}}$ is defined by $(1.1)$, where $\mathbb{N} \simeq\left(\mathbb{N}, \mathcal{E}_{\mathbb{N}}, m\right)$, with $\mathcal{E}_{\mathbb{N}}(n, n+1)=1$ and $m(n)=1$ for all $n \in \mathbb{N}$. Explicity, we have

$$
\Delta_{\mathbb{N}} f(n):=\left\{\begin{array}{cl}
2 f(n)-f(n-1)-f(n+1) & \text { if } n \geq 1, \\
f(n)-f(n+1) & \text { if } n=0,
\end{array} \quad \forall f \in \ell^{2}(\mathbb{N}, 1) .\right.
$$

We can express it with the help of $U$. Namely, we have:

$$
\Delta_{\mathbb{N}}=2-\left(U+U^{*}\right)-\mathbf{1}_{\{0\}}(\cdot) .
$$

A standard result is :

$$
\sigma_{\text {ess }}\left(\Delta_{\mathbb{N}}\right)=[0,4] \quad \text { and } \quad \sigma_{\mathrm{sc}}\left(\Delta_{\mathbb{N}}\right)=\emptyset
$$

We construct the conjugate operator in $\ell^{2}(\mathbb{N}, 1)$. On the space $\mathcal{C}_{c}(\mathbb{N})$, we define

$$
\begin{aligned}
\left.\mathcal{A}_{\mathbb{N}}\right|_{\mathcal{C}_{c}(\mathbb{N})} & :=\frac{1}{2}(S Q+Q S), \quad \text { where } S:=\frac{U-U^{*}}{2 \mathrm{i}} \\
& =\frac{\mathrm{i}}{2}\left(U\left(Q+\frac{1}{2}\right)-U^{*}\left(Q-\frac{1}{2}\right)\right) \\
& =-\frac{\mathrm{i}}{2}\left(\frac{1}{2}\left(U^{*}+U\right)+Q\left(U^{*}-U\right)\right) .
\end{aligned}
$$

We denote by $\mathcal{A}_{\mathbb{N}}$ its closure.

Lemma 3.2. The operator $\mathcal{A}_{\mathbb{N}}$ is essentially self-adjoint on $\mathcal{C}_{c}(\mathbb{N})$ and

$$
\mathcal{D}\left(\mathcal{A}_{\mathbb{N}}\right)=\mathcal{D}(Q S):=\left\{f \in \ell^{2}(\mathbb{N}), S f \in \mathcal{D}(Q)\right\}
$$

We refer to [GeGo] and [Mic, Lemma 5.7] for the essential self-adjointness and [GeGo, Lemma 3.1] for the domain.

We give a first technical lemma.

Lemma 3.3. On $\mathcal{C}_{c}(\mathbb{N})$, we have

$$
\begin{aligned}
& \left(U^{*}+U\right) \mathcal{A}_{\mathbb{N}}=-\frac{\mathrm{i}}{2}\left(\left(U^{2 *}-U^{2}\right) Q-\mathbf{1}-\frac{1}{2}\left(\mathbf{1}_{\{0\}}(\cdot)+U^{2}+U^{* 2}\right)\right), \\
& \mathcal{A}_{\mathbb{N}}\left(U^{*}+U\right)=\frac{\mathrm{i}}{2}\left(\left(U^{2}-U^{2 *}\right) Q-\frac{1}{2}\left(U^{2}+U^{* 2}\right)-\mathbf{1}-\frac{1}{2} \mathbf{1}_{\{0\}}(\cdot)\right) .
\end{aligned}
$$

Proof. We compute on $\mathcal{C}_{c}(\mathbb{N})$. The statement follows easily from

$$
\begin{aligned}
U \mathcal{A}_{\mathbb{N}} & =-\frac{\mathrm{i}}{2}\left(\left(\mathbf{1}_{\mathbb{N}}-U^{2}\right) Q-\frac{1}{2}\left(\mathbf{1}_{\mathbb{N}}+U^{2}\right),\right) . \\
U^{*} \mathcal{A}_{\mathbb{N}} & =-\frac{\mathrm{i}}{2}\left(\left(U^{*}-\mathbf{1}\right) Q-\frac{1}{2}\left(U^{* 2}-\frac{1}{2}\right)\right) .
\end{aligned}
$$

by taking the adjoint.

We can compute the first comutator. 
Lemma 3.4. The operator $\Delta_{\mathbb{N}}$ is $\mathcal{C}^{1}\left(\mathcal{A}_{\mathbb{N}}\right)$ and we have:

$$
\left[\Delta_{\mathbb{N}}, \mathrm{i} \mathcal{A}_{\mathbb{N}}\right]_{\circ}=\frac{1}{2} \Delta_{\mathbb{N}}\left(4-\Delta_{\mathbb{N}}\right)+K_{1}
$$

with $K_{1}$ a finite rank operator belonging to $\mathcal{C}^{\infty}(A)$.

This lemma is essentially given in [GeGo], see also [AlFr] for another type of presentation. For the convenience of the reader we reproduce it.

Proof. First, since $\delta_{\{0\}} \in \mathcal{D}\left(\mathcal{A}^{n}\right)$ for all $n \in \mathbb{N}, \delta_{\{0\}}$ and $K_{1}:=\left[\delta_{\{0\}}, \mathrm{i} \mathcal{A}_{\mathbb{N}}\right]_{\circ}$ belong to $\mathcal{C}^{1}\left(\mathcal{A}_{\mathbb{N}}\right)$ by Lemma 2.1. Next, we turn to the other part and work in the form sense and by density. Let $f \in \mathcal{C}_{c}(\mathbb{N})$. Since $\Delta_{\mathbb{N}} f \in \mathcal{C}_{c}(\mathbb{N})$ and using Lemma 3.3, we obtain:

$$
\begin{aligned}
\left\langle f,\left[\Delta_{\mathbb{N}}, \mathrm{i} \mathcal{A}_{\mathbb{N}}\right] f\right\rangle & :=\left\langle\Delta_{\mathbb{N}} f, \mathrm{i} \mathcal{A}_{\mathbb{N}} f\right\rangle-\left\langle-\mathrm{i} \mathcal{A}_{\mathbb{N}} f, \Delta_{\mathbb{N}} f\right\rangle \\
& =\mathrm{i}\left\langle f, \mathcal{A}_{\mathbb{N}}\left(U^{*}+U\right)-\left(U^{*}+U\right) \mathcal{A}_{\mathbb{N}} f\right\rangle+\left\langle f,\left[\delta_{\{0\}}, \mathrm{i} \mathcal{A}_{\mathbb{N}}\right] f\right\rangle \\
& =\frac{1}{2}\left\langle f, \Delta_{\mathbb{N}}\left(4-\Delta_{\mathbb{N}}\right) f\right\rangle+\left\langle f,\left[\delta_{\{0\}}, \mathrm{i} \mathcal{A}_{\mathbb{N}}\right]_{\circ} f\right\rangle .
\end{aligned}
$$

Since $\Delta_{\mathbb{N}}\left(4-\Delta_{\mathbb{N}}\right)$ and $\left[\delta_{\{0\}}, i \mathcal{A}_{\mathbb{N}}\right]_{\circ}$ are bounded operators and since $\mathcal{C}_{c}(\mathbb{N})$ is a core for $\mathcal{A}_{\mathbb{N}}$, there is a constant $c$ such that

$$
\left|\left\langle\Delta_{\mathbb{N}} f, \mathrm{i} \mathcal{A}_{\mathbb{N}} f\right\rangle-\left\langle-\mathrm{i} \mathcal{A}_{\mathbb{N}} f, \Delta_{\mathbb{N}} f\right\rangle\right| \leq c\|f\|^{2}, \text { for all } f \in \mathcal{D}(\mathcal{A}) .
$$

Hence, it is $\mathcal{C}^{1}\left(\mathcal{A}_{\mathbb{N}}\right)$. By density, we also obtain (3.7).

By induction, we infer:

Corollary 3.5. $\Delta_{\mathbb{N}} \in \mathcal{C}^{\infty}\left(\mathcal{A}_{\mathbb{N}}\right)$.

We mention [Mic] for an anisotropic use on $\mathbb{Z}$ based on the Mourre theory of $\Delta_{\mathbb{N}}$.

3.3. The funnel side. In this section we construct a conjugate operator for $\Delta_{\mathcal{G}^{\mathrm{f}}}$ and establish a Mourre estimate.

3.3.1. A first step into the analysis. As seen above, under the identification

$$
\ell^{2}\left(\mathcal{V}^{\mathrm{f}}, m\right)=\ell^{2}\left(\mathbb{N}, m_{1}^{\mathrm{f}}\right) \otimes \ell^{2}\left(\mathcal{V}_{2}^{\mathrm{f}}, m_{2}^{\mathrm{f}}\right) .
$$

We have

$$
\Delta_{\mathcal{G}^{\mathrm{f}}}:=\Delta_{\mathcal{G}_{1}^{\mathrm{f}}} \otimes \frac{1}{m_{2}^{\mathrm{f}}}+\frac{1}{m_{1}^{\mathrm{f}}(\cdot)} \otimes \Delta_{\mathcal{G}_{2}^{\mathrm{f}}} .
$$

Recall here that $m_{2}$ is a constant. The first remark is that

Lemma 3.6.

$$
\frac{1}{m_{1}^{\mathrm{f}}(\cdot)} \otimes \Delta_{\mathcal{G}_{2}^{\mathrm{f}}} \in \mathcal{K}\left(\ell^{2}\left(\mathcal{V}^{\mathrm{f}}\right)\right)
$$

Proof. Note that $\Delta_{\mathcal{G}_{2}^{\mathrm{f}}}$ is of finite rank since $\mathcal{V}_{2}$ is finite and that $\frac{1}{m_{1}^{\mathrm{f}}(\cdot)}$ is a compact operator since $m_{1}^{\mathrm{f}}(n) \rightarrow \infty$, as $n \rightarrow \infty$.

Since $m_{2}$ is constant and $\operatorname{deg}_{\mathcal{G}_{1}^{\mathrm{f}}}$ is bounded, we obtain:

Proposition 3.7. We have $\Delta_{\mathcal{G}^{\mathrm{f}}} \in \mathcal{B}\left(\ell^{2}\left(\mathcal{V}^{\mathrm{f}}\right), m^{\mathrm{f}}\right)$.

Recalling the Proposition 3.1, we obtain:

$$
T_{1 \rightarrow m_{1}^{\mathrm{f}}}^{-1} \Delta_{\mathcal{G}_{1}^{\mathrm{f}}} T_{1 \rightarrow m_{1}^{\mathrm{f}}}=\Delta_{\mathbb{N}}+\left(e^{1 / 2}-1\right) \mathbf{1}_{\{0\}}+e^{1 / 2}+e^{-1 / 2}-2
$$

Recalling Lemma 3.6, we infer immediately

$$
\sigma_{\text {ess }}\left(\Delta_{\mathcal{G}^{\mathrm{f}}}\right)=\left[\frac{\alpha}{m_{2}}, \frac{\beta}{m_{2}}\right] \quad \text { and } \quad \sigma_{\mathrm{sc}}\left(\Delta_{\mathcal{G}_{1}^{\mathrm{f}}}\right)=\emptyset,
$$

with $\alpha$ and $\beta$ are given in (1.2). 
3.3.2. Construction of the conjugate operator. In order to get also $\sigma_{\mathrm{sc}}\left(\Delta_{\mathcal{G}^{\mathrm{f}}}\right)=\emptyset$, we rely on the Mourre theory and construct a conjugate operator for $\Delta_{\mathcal{G}^{\mathrm{f}}}$. Recalling (3.6) and with respect to (3.8), we set

$$
\mathcal{A}_{\mathcal{G}^{\mathrm{f}}}:=\mathcal{A}_{m_{1}^{\mathrm{f}}} \otimes 1_{\mathcal{V}_{2}^{\mathrm{f}}}:=T_{1 \rightarrow m_{1}^{\mathrm{f}}} \mathcal{A}_{\mathbb{N}} T_{1 \rightarrow m_{1}^{\mathrm{f}}}^{-1} \otimes 1_{\mathcal{V}_{2}^{\mathrm{f}}} .
$$

It is essentially self-adjoint on $\mathcal{C}_{c}\left(\mathcal{V}^{\mathrm{f}}\right)$ and on $\mathcal{C}_{c}(\mathbb{N}) \otimes \ell^{2}\left(\mathcal{V}^{\mathrm{f}}\right)$ by Lemma 3.2. It acts as follows:

Proposition 3.8. On $\mathcal{C}_{c}(\mathbb{N})$, we have

$$
\mathcal{A}_{m_{1}^{\mathrm{f}}}=\frac{\mathrm{i}}{2}\left(e^{1 / 2}(Q-1 / 2) U-e^{-1 / 2}(Q+1 / 2) U^{*}\right) .
$$

Proof. Let $f \in \mathcal{C}_{c}(\mathbb{N})$,

$$
\begin{aligned}
& \mathcal{A}_{m_{1}^{\mathrm{f}}} f(n)=-\frac{\mathrm{i}}{2 \sqrt{m_{1}^{\mathrm{f}}(n)}}\left(\frac{1}{2}\left(U+U^{*}\right)+Q\left(U^{*}-U\right)\right) T_{1 \rightarrow m_{1}^{\mathrm{f}}}^{-1} f(n) \\
& =\frac{\mathrm{i}}{2}\left(\left(n-\frac{1}{2}\right) \sqrt{\frac{m_{1}^{\mathrm{f}}(n-1)}{m_{1}^{\mathrm{f}}(n)}} f(n-1)-\left(n+\frac{1}{2}\right) \sqrt{\frac{m_{1}^{\mathrm{f}}(n+1)}{m_{1}^{\mathrm{f}}(n)}} f(n+1)\right) \\
& =\frac{\mathrm{i}}{2}\left(e^{1 / 2}\left(n-\frac{1}{2}\right) U f(n)-e^{-1 / 2}\left(n+\frac{1}{2}\right) U^{*} f(n)\right) .
\end{aligned}
$$

This concludes the proof.

We turn to the regularity. In order to lighten the computation, given a graph $\mathcal{G}=(\mathcal{E}, \mathcal{V}, m)$, we write

$T_{1} \simeq T_{2}$ if there is $K: \mathcal{C}_{c}(\mathcal{V}) \rightarrow \mathcal{C}_{c}(\mathcal{V})$ of finite rank such that $T_{1}=T_{2}+K$

Thanks to Lemma 2.1 and Proposition 3.8, we obtain immediately:

Lemma 3.9. Assume that $T_{1} \simeq T_{2}$. Then for all $n \in \mathbb{N}$,

$$
T_{1} \in \mathcal{D}\left(\mathcal{A}_{\mathcal{G}^{\mathrm{f}}}^{n}\right) \Leftrightarrow T_{2} \in \mathcal{D}\left(\mathcal{A}_{\mathcal{G}^{\mathrm{f}}}^{n}\right) .
$$

We have:

Lemma 3.10. We have $\Delta_{\mathcal{G}^{\mathrm{f}}} \in \mathcal{C}^{1}\left(\mathcal{A}_{\mathcal{G}^{\mathrm{f}}}\right)$ and

$$
\left[\Delta_{\mathcal{G}^{\mathrm{f}}}, \mathrm{i} \mathcal{A}_{\mathcal{G}^{\mathrm{f}}}\right]_{\circ}=w\left(\Delta_{\mathcal{G}^{\mathrm{f}}}\right)+K
$$

where

$$
w^{\mathrm{f}}(x):=\frac{m_{2}}{2}\left(x-\frac{\alpha}{m_{2}}\right)\left(\frac{\beta}{m_{2}}-x\right),
$$

with $\alpha$ and $\beta$ as in (1.2) and $K$ is a compact operator.

Proof. We prove that $\left[\Delta_{\mathcal{G}^{\mathrm{f}}}, \mathrm{i} \mathcal{A}_{\mathcal{G}^{\mathrm{f}}}\right]_{\circ} \in \mathcal{B}\left(\ell^{2}\left(\mathcal{V}^{\mathrm{f}}, m^{\mathrm{f}}\right)\right)$. As in Lemma 3.4 and working in the form sense on $\mathcal{C}_{c}(\mathbb{N}) \otimes \ell^{2}\left(\mathcal{V}_{2}^{\mathrm{f}}\right)$, a straightforward computation leads to

$$
\begin{aligned}
{\left[\Delta_{\mathcal{G}_{1}^{\mathrm{f}}} \otimes\right.} & \left.\frac{1}{m_{2}}, \mathrm{i} \mathcal{A}_{\mathcal{G}^{\mathrm{f}}}\right] \simeq \frac{1}{2}\left(\Delta_{\mathcal{G}_{1}^{\mathrm{f}}}-\alpha\right)\left(\beta-\Delta_{\mathcal{G}_{1}^{\mathrm{f}}}\right) \otimes \frac{1}{m_{2}} \\
\simeq & w^{\mathrm{f}}\left(\Delta_{\mathcal{G}^{\mathrm{f}}}\right)-\frac{m_{2}}{2}\left(\frac{1}{m_{1}(\cdot)} \otimes \Delta_{\mathcal{G}_{2}^{\mathrm{f}}}\right)\left(\frac{\beta}{m_{2}}-\Delta_{\mathcal{G}_{1}^{\mathrm{f}}} \otimes \frac{1}{m_{2}}-\frac{1}{m_{1}(\cdot)} \otimes \Delta_{\mathcal{G}_{2}^{\mathrm{f}}}\right) \\
& +\frac{m_{2}}{2}\left(\Delta_{\mathcal{G}_{1}^{\mathrm{f}}} \otimes \frac{1}{m_{2}}-\frac{\alpha}{m_{2}}\right)\left(\frac{1}{m_{1}(\cdot)} \otimes \Delta_{\mathcal{G}_{2}^{\mathrm{f}}}\right) . \\
= & w^{\mathrm{f}}\left(\Delta_{\mathcal{G}^{\mathrm{f}}}\right)+K^{\prime}
\end{aligned}
$$


where $K^{\prime}$ is a compact operator coming from Lemma 3.6 and Lemma 2.1. We turn to the second part of $\Delta_{\mathcal{G}^{\mathrm{f}}}$.

$$
\begin{aligned}
{\left[\frac{1}{m_{1}^{\mathrm{f}}(\cdot)}, \mathrm{i} \mathcal{A}_{m_{1}^{\mathrm{f}}}\right] \otimes \Delta_{\mathcal{G}_{2}^{\mathrm{f}}} } & =T_{1 \rightarrow m_{1}^{\mathrm{f}}}\left[\frac{1}{m_{1}^{\mathrm{f}}(\cdot)}, \mathrm{i} \mathcal{A}_{\mathbb{N}}\right] T_{1 \rightarrow m_{1}^{\mathrm{f}}}^{-1} \otimes \Delta_{\mathcal{G}_{2}^{\mathrm{f}}} \\
& =T_{1 \rightarrow m_{1}^{\mathrm{f}}}\left(\frac{1}{2}(e-1) e^{-Q}\left(Q-\frac{1}{2}\right) U\right) T_{1 \rightarrow m_{1}^{\mathrm{f}}}^{-1} \otimes \Delta_{\mathcal{G}_{2}^{\mathrm{f}}},
\end{aligned}
$$

in the form sense on $\mathcal{C}_{c}(\mathbb{N}) \otimes \ell^{2}\left(\mathcal{V}_{2}^{\mathrm{f}}\right)$. The operator is a compact since $U$ is bounded and $\lim _{n \rightarrow \infty} e^{-n}(n-1 / 2)=0$.

This implies that $\left[\Delta_{\mathcal{G}^{\mathrm{f}}}, \mathrm{i} \mathcal{A}_{\mathcal{G}^{\mathrm{f}}}\right]_{\circ} \in \mathcal{B}\left(\ell^{2}\left(\mathcal{V}^{\mathrm{f}}, m^{\mathrm{f}}\right)\right)$ and that (3.11) holds true. Finally, since $\mathcal{C}_{c}(\mathbb{N}) \otimes \ell^{2}\left(\mathcal{V}_{2}^{\mathrm{f}}\right)$ is a core for $\mathcal{A}_{\mathcal{G}^{\mathrm{f}}}$, we deduce that $\Delta_{\mathcal{G}^{\mathrm{f}}} \in \mathcal{C}^{1}\left(\mathcal{A}_{\mathcal{G}^{\mathrm{f}}}\right)$.

Lemma 3.11. We have $\Delta_{\mathcal{G}^{\mathrm{f}}} \in \mathcal{C}^{2}\left(\mathcal{A}_{\mathcal{G}^{\mathrm{f}}}\right)$.

Proof. As above, since $\mathcal{C}_{c}(\mathbb{N}) \otimes \ell^{2}\left(\mathcal{V}_{2}^{\mathrm{f}}\right)$ is a core for $\mathcal{A}_{\mathcal{G}^{\mathrm{f}}}$ it is enough to prove that $\left[\left[\Delta_{\mathcal{G}^{\mathrm{f}}}, \mathrm{i} \mathcal{A}_{\mathcal{G}^{\mathrm{f}}}\right]_{\mathrm{o}}, \mathrm{i} \mathcal{A}_{\mathcal{G}^{\mathrm{f}}}\right]$, defined initially in the form sense on $\mathcal{C}_{c}(\mathbb{N}) \otimes \ell^{2}\left(\mathcal{V}_{2}^{\mathrm{f}}\right)$, extends to an element of $\mathcal{B}\left(\ell^{2}\left(\mathcal{V}^{\mathrm{f}}, m^{\mathrm{f}}\right)\right)$.

We prove that the right hand side of (3.11) belongs to $\mathcal{C}^{1}\left(A_{\mathcal{G}_{\mathrm{f}}}\right)$. It composed of $w\left(\Delta_{\mathcal{G}_{\mathrm{f}}}\right)$ which is $\mathcal{C}^{1}\left(A_{\mathcal{G}_{\mathrm{f}}}\right)$ (as product of bounded operators belonging to $\mathcal{C}^{1}\left(A_{\mathcal{G}_{\mathrm{f}}}\right)$ ), terms with finite support that are also in $\mathcal{C}^{1}\left(A_{\mathcal{G}_{\mathrm{f}}}\right)$ by Lemma 2.1 and terms similar to (3.13). Therefore $\left[\left[\Delta_{\mathcal{G}_{1}^{\mathrm{f}}} \otimes \frac{1}{m_{2}}, \mathrm{i} \mathcal{A}_{\mathcal{G}^{\mathrm{f}}}\right]_{\circ}, \mathrm{i} \mathcal{A}_{\mathcal{G}^{\mathrm{f}}}\right]$ extends to a bounded operator.

We turn to the second part. It remains to show that the left hand side of (3.13) belongs to $\mathcal{C}^{1}\left(A_{\mathcal{G}_{\mathrm{f}}}\right)$. Repeating the computation done in (3.13), we see that since $\lim _{n \rightarrow \infty} e^{-n}\langle n\rangle^{2}=0,\left[\left[\frac{1}{m_{1}^{\mathrm{f}}(\cdot)}, \mathrm{i} \mathcal{A}_{m_{1}^{\mathrm{f}}}\right]_{\circ}, \mathcal{A}_{m_{1}^{\mathrm{f}}}\right]$ extends to a compact operator.

Remark 3.12. By induction, we can prove that $\Delta_{\mathcal{G}^{\mathrm{f}}} \in \mathcal{C}^{\infty}\left(\mathcal{A}_{\mathcal{G}^{\mathrm{f}}}\right)$.

Finally, we establish the Mourre estimate.

Proposition 3.13. We have $\Delta_{\mathcal{G}^{\mathrm{f}}} \in \mathcal{C}^{2}\left(\mathcal{A}_{\mathcal{G}^{\mathrm{f}}}\right)$. Given a compact interval $\mathcal{I} \subset$ $\left(\alpha / m_{2}, \beta / m_{2}\right)$, there are $c>0$, a compact operator $K$ such that

$$
E_{\mathcal{I}}\left(\Delta_{\mathcal{G}^{\mathrm{f}}}\right)\left[\Delta_{\mathcal{G}^{\mathrm{f}}}, \mathrm{i} \mathcal{A}_{\mathcal{G}^{\mathrm{f}}}\right]_{\circ} E_{\mathcal{I}}\left(\Delta_{\mathcal{G}^{\mathrm{f}}}\right) \geq c E_{\mathcal{I}}\left(\Delta_{\mathcal{G}^{\mathrm{f}}}\right)+K,
$$

in the form sense. In particular, $\sigma_{\mathrm{sc}}\left(\Delta_{\mathcal{G}^{\mathrm{f}}}\right)=\emptyset$.

Proof. Lemma 3.11 gives that $\Delta_{\mathcal{G}^{\mathrm{f}}} \in \mathcal{C}^{2}\left(\mathcal{A}_{\mathcal{G}^{\mathrm{f}}}\right)$. By $(3.11)$, we obtain

$$
\begin{aligned}
E_{\mathcal{I}}\left(\Delta_{\mathcal{G}^{\mathrm{f}}}\right)\left[\Delta_{\mathcal{G}^{\mathrm{f}}}, \mathrm{i} \mathcal{A}_{\mathcal{G}^{\mathrm{f}}}\right]_{\circ} E_{\mathcal{I}}\left(\Delta_{\mathcal{G}^{\mathrm{f}}}\right) & =E_{\mathcal{I}}\left(\Delta_{\mathcal{G}^{\mathrm{f}}}\right) w\left(\Delta_{\mathcal{G}^{\mathrm{f}}}\right) E_{\mathcal{I}}\left(\Delta_{\mathcal{G}^{\mathrm{f}}}\right)+K \\
& \geq c E_{\mathcal{I}}\left(\Delta_{\mathcal{G}^{\mathrm{f}}}\right)+K,
\end{aligned}
$$

where $K$ is a compact operator and

$$
c:=\frac{m_{2}}{2} \inf _{x \in I}\left(x-\frac{\alpha}{m_{2}}\right)\left(\frac{\beta}{m_{2}}-x\right)>0 .
$$

The absence of singular continuous spectrum follows from the general theory.

To lighten the text we did not expand more consequences of the Mourre theory in this case and refer to Theorem 4.3 for them.

3.4. The cusps side. In this section we construct a conjugate operator for $\Delta_{\mathcal{G}^{\mathrm{c}}}$ and establish a Mourre estimate. By contrast with the funnel side, we shall refine the tensor product decomposition. 
3.4.1. The model and the low/high energy decomposition. Again we rely on the decomposition

$$
\ell^{2}\left(\mathcal{V}^{\mathrm{c}}, m\right)=\ell^{2}\left(\mathbb{N}, m_{1}^{\mathrm{c}}\right) \otimes \ell^{2}\left(\mathcal{V}_{2}^{\mathrm{c}}, m_{2}\right)
$$

We have

$$
\Delta_{\mathcal{G}^{\mathrm{c}}}:=\Delta_{\mathcal{G}_{1}^{\mathrm{c}}} \otimes \frac{1}{m_{2}}+\frac{1}{m_{1}^{\mathrm{c}}(\cdot)} \otimes \Delta_{\mathcal{G}_{2}^{\mathrm{c}}} .
$$

Recall that $m_{2}$ is a constant. Unlike with the treatment of $\Delta_{\mathcal{G}^{\mathrm{f}}}$, we refine the tensor product decomposition. In the spirit of [GoMo, GoTr], we denote by $P^{\text {le }}$ the projection on $\operatorname{ker}\left(\Delta_{\mathcal{G}_{2}}\right)$ and by $P^{\text {he }}$ is the projection on $\operatorname{ker}\left(\Delta_{\mathcal{G}_{2}}\right)^{\perp}$. Here, le stands for low energy and he for high energy. We shall take advantage of

$$
\begin{aligned}
\ell^{2}\left(\mathcal{V}^{\mathrm{c}}, m\right) & :=\mathcal{H}^{\mathrm{le}} \oplus \mathcal{H}^{\mathrm{he}} \\
& :=\ell^{2}\left(\mathbb{N}, m_{1}^{\mathrm{c}}\right) \otimes \operatorname{ker}\left(\Delta_{2}\right) \oplus \ell^{2}\left(\mathbb{N}, m_{1}^{\mathrm{c}}\right) \otimes \operatorname{ker}\left(\Delta_{2}\right)^{\perp} .
\end{aligned}
$$

The main idea is the continuous spectrum comes from the low energy part of the space whereas the discrete spectrum arises from the high energy part.

We have that $\Delta_{\mathcal{G}^{c}}:=\Delta_{\mathcal{G}^{c}}^{\mathrm{le}} \oplus \Delta_{\mathcal{G}^{c}}^{\text {he }}$, where

$$
\Delta_{\mathcal{G}^{\mathrm{c}}}^{\mathrm{le}}:=\Delta_{\mathcal{G}_{1}^{\mathrm{c}}} \otimes \frac{1}{m_{2}} P^{\mathrm{le}}
$$

on $\left(1 \otimes P^{\mathrm{le}}\right) \ell^{2}\left(\mathcal{V}^{\mathrm{c}}, m^{\mathrm{c}}\right)$, and

$$
\Delta_{\mathcal{G}^{\mathrm{c}}}^{\mathrm{he}}:=\Delta_{\mathcal{G}_{1}^{\mathrm{c}}} \otimes \frac{1}{m_{2}} P^{\text {he }}+\frac{1}{m_{1}^{\mathrm{c}}(\cdot)} \otimes P^{\mathrm{he}} \Delta_{\mathcal{G}_{2}^{\mathrm{c}}},
$$

on $\left(1 \otimes P^{\text {he }}\right) \ell^{2}\left(\mathcal{V}^{\mathrm{c}}, m^{\mathrm{c}}\right)$. We stress that $m_{2}$ is constant.

Unlike $\Delta_{\mathcal{G}^{\mathrm{f}}}, \Delta_{\mathcal{G}^{\mathrm{c}}}$ is unbounded. More precisely we have:

Proposition 3.14. The operator $\Delta_{\mathcal{G}^{c}}$ is essentially self-adjoint on $\mathcal{C}_{c}(\mathbb{N}) \otimes \ell^{2}\left(\mathcal{V}_{2}\right)$ and on $\mathcal{C}_{c}\left(\mathcal{V}^{c}\right)$. Its domain is given by $\mathcal{D}\left(\frac{1}{m_{1}^{c}(\cdot)} \otimes \Delta_{\mathcal{G}_{2}^{c}}\right)$.

Proof. First $m_{1}^{c}(\cdot)$ is essentially self-adjoint of $\mathcal{C}_{c}(\mathbb{N})$. Since $\Delta_{\mathcal{G}_{2}^{c}}$ is bounded, we infer that $\frac{1}{m_{1}^{c}(\cdot)} \otimes \Delta_{\mathcal{G}_{2}^{c}}$ is essentially self-adjoint on $\mathcal{C}_{c}(\mathbb{N}) \otimes \ell^{2}\left(\mathcal{V}_{2}\right)$. Next, since $\Delta_{\mathcal{G}_{1}^{c}} \otimes \frac{1}{m_{2}}$ is bounded, $\Delta_{\mathcal{G}^{c}}$ is essentially self-adjoint on $\mathcal{C}_{c}(\mathbb{N}) \otimes \ell^{2}\left(\mathcal{V}_{2}\right)$ by the KatoRellich Theorem, e.g., [ReSi, Theorem X.12]. The statement with $\mathcal{C}_{c}\left(\mathcal{V}^{\mathrm{c}}\right)$ follows by standard approximations.

Using the notation given in (3.3), we see that:

$$
T_{m_{1}^{c} \rightarrow 1} \Delta_{\mathcal{G}_{1}^{c}} T_{m_{1}^{c} \rightarrow 1}^{-1}=\Delta_{\mathbb{N}}-\left(e^{-1 / 2}-1\right) \mathbf{1}_{\{0\}}(\cdot)+e^{1 / 2}+e^{-1 / 2}-2 \text { in } \ell^{2}(\mathbb{N}) .
$$

By using for instance some Jacobi matrices techniques, it is well-known that the essential spectrum of $\Delta_{\mathcal{G}^{c}}^{\text {le }}$ is purely absolutely continuous and

$$
\sigma_{\mathrm{ac}}\left(\Delta_{\mathcal{G}^{\mathrm{c}}}^{\mathrm{le}}\right)=[\alpha, \beta],
$$

with multiplicity one, e.g., [We]. Recall that $\alpha$ and $\beta$ are defined in (1.2).

We turn to the high energy part. Using [GoTr, Equation (10)],

$$
\frac{1}{m_{1}^{\mathrm{c}}(\cdot)} \otimes \Delta_{\mathcal{G}_{2}^{\mathrm{c}}} P^{\mathrm{he}} \leq \Delta_{\mathcal{G}^{\mathrm{c}}}\left(1 \otimes P^{\mathrm{he}}\right) \leq 2 M+\frac{1}{m_{1}^{\mathrm{c}}(\cdot)} \otimes \Delta_{\mathcal{G}_{2}^{\mathrm{c}}} P^{\text {he }}
$$

Using the min-max Theorem and since $m_{1}^{\mathrm{c}}(n) \rightarrow 0$ as $n \rightarrow \infty, \Delta_{\mathcal{G}^{\mathrm{c}}}\left(1 \otimes P^{\text {he }}\right)$ has a compact resolvent. We infer that

$$
\sigma_{\mathrm{ac}}\left(\Delta_{\mathcal{G}^{\mathrm{c}}}\right)=\left[\frac{\alpha}{m_{2}}, \frac{\beta}{m_{2}}\right] \text { and } \sigma_{\mathrm{sc}}\left(\Delta_{\mathcal{G}^{c}}\right)=\emptyset
$$


3.4.2. The conjugate operator. We pursue the analysis of $\Delta_{\mathcal{G}}$ in order to apply the Mourre theory to it. We go back to $\ell^{2}\left(\mathbb{N}, m_{1}^{\mathrm{c}}\right) \otimes \operatorname{ker}\left(\Delta_{2}\right)$. We set:

$$
\mathcal{A}_{\mathcal{G}^{\mathrm{c}}}^{\mathrm{le}}:=T_{m_{1}^{\mathrm{c}} \rightarrow 1}^{-1} \mathcal{A}_{\mathbb{N}} T_{m_{1}^{\mathrm{c}} \rightarrow 1} \otimes P^{\mathrm{le}} .
$$

It is self-adjoint. Straightforwardly we get

$$
\mathcal{A}_{\mathcal{G}^{\mathrm{c}}}^{\mathrm{le}}=-\frac{\mathrm{i}}{2}\left(e^{-1 / 2}\left(Q+\frac{1}{2}\right) U^{*}+e^{1 / 2}\left(\frac{1}{2}-Q\right) U\right) \otimes P^{\mathrm{le}}
$$

on $\mathcal{C}_{c}(\mathbb{N}) \otimes \operatorname{ker}\left(\Delta_{2}\right)$. With respect to $(3.17)$, we set

$$
\mathcal{A}_{\mathcal{G}^{\mathrm{c}}}:=\mathcal{A}_{\mathcal{G}^{\mathrm{c}}}^{\text {le }} \oplus \mathcal{A}_{\mathcal{G}^{\mathrm{c}}}^{\text {he }} \text {, where } \mathcal{A}_{\mathcal{G}^{\mathrm{c}}}^{\text {he }}:=0 \text {. }
$$

By Lemma 3.2, it is essentially self-adjoint on $\mathcal{C}_{c}(\mathbb{N}) \otimes \ell^{2}\left(\mathcal{V}_{2}^{\mathrm{c}}, m_{2}\right)$ and also on $\mathcal{C}_{c}\left(\mathcal{V}^{\mathrm{c}}\right)$ by standard approximation. Keeping the notation of Lemma 3.9, we obtain:

Lemma 3.15. We have $\Delta_{\mathcal{G}^{\mathrm{c}}} \in \mathcal{C}^{1}\left(\mathcal{A}_{\mathcal{G}^{\mathrm{c}}}\right)$ and

$$
\left[\Delta_{\mathcal{G}^{\mathrm{c}}}, \mathrm{i} \mathcal{A}_{\mathcal{G}^{\mathrm{c}}}\right]_{\circ} \simeq w^{\mathrm{c}}\left(\Delta_{\mathcal{G}^{\mathrm{c}}}^{\mathrm{le}}\right) \oplus 0
$$

with respect to (3.17), with $K \in \mathcal{K}\left(\ell^{2}\left(\mathcal{V}^{\mathrm{c}}, m^{\mathrm{c}}\right)\right)$ and

$$
w^{\mathrm{c}}(x):=\frac{m_{2}}{2}\left(x-\frac{\alpha}{m_{2}}\right)\left(\frac{\beta}{m_{2}}-x\right) .
$$

In particular, $\left[\Delta_{\mathcal{G}^{\mathrm{c}}}, \mathrm{i} \mathcal{A}_{\mathcal{G}^{\mathrm{c}}}\right]_{\circ} \in \mathcal{B}\left(\ell^{2}\left(\mathcal{V}^{\mathrm{c}}, m^{\mathrm{c}}\right)\right)$.

Proof. As in Lemma 3.4, using Lemma 2.1, and working in the form sense on $\mathcal{C}_{c}(\mathbb{N}) \otimes \ell^{2}\left(\mathcal{V}_{2}^{c}\right)$, a straightforward computation leads to

$$
\begin{aligned}
{\left[\Delta_{\mathcal{G}_{1}^{\mathrm{c}}} \otimes \frac{1}{m_{2}^{c}}, \mathrm{i} \mathcal{A}_{\mathcal{G}^{\mathrm{c}}}\right] } & \simeq \frac{1}{2}\left(\Delta_{\mathcal{G}_{1}^{\mathrm{c}}}-\alpha\right)\left(\beta-\Delta_{\mathcal{G}_{1}^{\mathrm{c}}}\right) \otimes \frac{1}{m_{2}} P^{\mathrm{le}} \\
& \simeq w^{c}\left(\Delta_{\mathcal{G}^{\mathrm{f}}}^{\mathrm{le}}\right) \oplus 0 .
\end{aligned}
$$

We turn to the second part of $\Delta_{\mathcal{G}^{c}}$.

$$
\left[\frac{1}{m_{1}^{\mathrm{c}}(\cdot)} \otimes \Delta_{\mathcal{G}_{2}^{\mathrm{f}}}, \mathrm{i}_{\mathcal{G}_{\mathcal{G}^{\mathrm{c}}}}\right]=\left[\frac{1}{m_{1}^{\mathrm{c}}(\cdot)}, \mathcal{A}_{\mathcal{G}_{1}^{\mathrm{c}}}\right] \otimes 0=0
$$

This implies that $\left[\Delta_{\mathcal{G}^{c}}, \mathrm{i} \mathcal{A}_{\mathcal{G}^{c}}\right]_{\circ} \in \mathcal{B}\left(\ell^{2}\left(\mathcal{V}^{\mathrm{c}}, m^{\mathrm{c}}\right)\right)$ and (3.21).

It remains to prove that $\Delta_{\mathcal{G}^{c}} \in \mathcal{C}^{1}\left(\mathcal{A}_{\mathcal{G}^{c}}\right)$. We check the hypotheses of Lemma 2.3. Let $\left\{\mathcal{X}_{n}\right\}_{n \in \mathbb{N}}$ be a family of functions defined on $\mathcal{V}_{1}^{\mathrm{c}} \times \mathcal{V}_{2}^{\mathrm{c}}$ as follows:

$$
\mathcal{X}_{n}\left(x_{1}, x_{2}\right):=\left(\left(1-\frac{x_{1}-n}{n^{2}+1}\right) \vee 0\right) \wedge 1 .
$$

Note that $\operatorname{supp}\left(\mathcal{X}_{n}\right)=\llbracket 0, n^{2}+n \rrbracket \times \mathcal{V}_{2}$ and $\forall\left(x_{1}, x_{2}\right) \in \llbracket 0, n \rrbracket \times \mathcal{V}_{2}^{\mathrm{c}}, \quad \mathcal{X}_{n}\left(x_{1}, x_{2}\right)=1$. We set $\mathscr{D}:=\mathcal{C}_{c}\left(\mathcal{V}^{\mathrm{c}}\right)$.

1) We have $\left\|\mathcal{X}_{n}\right\|_{\infty}=1$ then $\left\|\mathcal{X}_{n}(\cdot)\right\|_{\mathcal{B}\left(\ell^{2}\left(\mathcal{V}^{c}, m^{c}\right)\right)}=1$. Moreover, $\mathcal{X}_{n}(\cdot)$ tends strongly to 1 as $n \rightarrow+\infty$. Now, we shall show that $\sup _{n}\left\|\mathcal{X}_{n}(\cdot)\right\|_{\mathcal{D}\left(\Delta_{\mathcal{G}^{\mathrm{f}}}\right)}<\infty$. Since

$$
\left[\Delta_{\mathcal{G}_{1}^{\mathrm{c}}} \otimes \frac{1}{m_{2}}+\frac{1}{m_{1}^{\mathrm{c}}(\cdot)} \otimes \Delta_{\mathcal{G}_{2}^{\mathrm{c}}}, \mathcal{X}_{n}(\cdot)\right]=\underbrace{\left[\Delta_{\mathcal{G}_{1}^{\mathrm{c}}}, \mathcal{X}_{n}(\cdot)\right] \otimes \frac{1}{m_{2}}}_{\text {bounded by } 2\left\|\Delta_{\mathcal{G}_{1}^{c}}\right\| / m_{2}}+\underbrace{\left[\frac{1}{m_{1}^{\mathrm{c}}(\cdot)}, \mathcal{X}_{n}(\cdot)\right]}_{=0} \otimes \Delta_{2},
$$

then there is $c>0$ such that, for all $f \in \mathcal{C}_{c}\left(\mathcal{V}^{c}\right)$ such that $f \in\left(\Delta_{\mathcal{G}^{c}}+\mathrm{i}\right) \mathcal{C}_{c}\left(\mathcal{V}^{\mathrm{c}}\right)$ and $n \in \mathbb{N}$,

$$
\left\|\left(\Delta_{\mathcal{G}^{\mathrm{c}}}+\mathrm{i}\right) \mathcal{X}_{n}(Q)\left(\Delta_{\mathcal{G}^{\mathrm{c}}}+\mathrm{i}\right)^{-1} f\right\| \leq c\|f\| .
$$

Since $\Delta_{\mathcal{G}^{c}}$ is essentially self-adjoint on $\mathcal{C}_{c}\left(\mathcal{V}^{c}\right)$ and since $-\mathrm{i} \notin \sigma\left(\Delta_{\mathcal{G}^{c}}\right)$, it holds for all $f \in \ell^{2}\left(\mathcal{V}^{\mathrm{c}}, m^{\mathrm{c}}\right)$. In particular, we derive that $\left\|\left(\Delta_{\mathcal{G}^{\mathrm{c}}}+\mathrm{i}\right) \mathcal{X}_{n}(Q) f\right\| \leq c\left\|\left(\Delta_{\mathcal{G}^{\mathrm{c}}}+\mathrm{i}\right) f\right\|$, for all $f \in \ell^{2}\left(\mathcal{V}^{\mathrm{c}}, m^{\mathrm{c}}\right)$. In particular, $\sup _{n}\left\|\mathcal{X}_{n}(\cdot)\right\|_{\mathcal{D}\left(\Delta_{\mathcal{C}^{\mathrm{f}}}\right)}<\infty$.

2) Given $f \in \mathcal{C}_{c}\left(\mathcal{V}^{c}\right)$, note that for $n$ large enough $\mathcal{X}_{n}(\cdot) f=f$. In particular, for all $f \in \mathcal{C}_{c}\left(\mathcal{V}^{c}\right), \mathcal{A}_{\mathcal{G}^{c}} \mathcal{X}_{n}(\cdot) f \rightarrow \mathcal{A}_{\mathcal{G}^{c}} f$, as $n \rightarrow \infty$. 
3) Noticing that $\left[\Delta_{\mathcal{G}^{\mathrm{c}}}, \mathcal{X}_{n}(\cdot)\right]=\left[\Delta_{\mathcal{G}_{1}^{\mathrm{c}}}, \mathcal{X}_{n}(\cdot)\right] \otimes \frac{1}{m_{2}}$, a straightforward computation ensures that there exists $c$ such that

$$
\left\|\mathcal{A}_{\mathcal{G}^{\mathrm{c}}}\left[\Delta_{\mathcal{G}^{\mathrm{c}}}, \mathcal{X}_{n}(\cdot)\right]\right\| \leq \frac{c}{\langle n\rangle} .
$$

Finally for all $z \in \mathbb{C} \backslash \mathbb{R}$, the condition $\mathcal{X}_{n}(\cdot)\left(\Delta_{\mathcal{G}^{\mathrm{c}}}-z\right)^{-1} \mathcal{C}_{c}\left(\mathcal{V}^{\mathrm{c}}\right) \subset \mathcal{C}_{c}\left(\mathcal{V}^{\mathrm{c}}\right)$ is immediate as $\mathcal{X}_{n}$ is with finite support. [GoMo, Lemma A.2] gives that $\Delta_{\mathcal{G}^{\mathrm{c}}}^{\mathrm{le}} \in \mathcal{C}^{1}\left(\mathcal{A}_{\mathcal{G}^{\mathrm{c}}}^{\mathrm{le}}\right)$.

Lemma 3.16. We have $e^{\mathrm{i} t \mathcal{A}_{\mathcal{G}^{c}}} \mathcal{D}\left(\Delta_{\mathcal{G}^{\mathrm{c}}}\right) \subset \mathcal{D}\left(\Delta_{\mathcal{G}^{\mathrm{c}}}\right)$ for all $t \in \mathbb{R}$.

Proof. We have $\Delta_{\mathcal{G}^{\mathrm{c}}} \in \mathcal{C}^{1}\left(\mathcal{A}_{\mathcal{G}^{\mathrm{c}}}\right)$ and $\left[\Delta_{\mathcal{G}^{\mathrm{c}}}, \mathrm{i} \mathcal{A}_{\mathcal{G}^{\mathrm{c}}}\right]_{\circ}$ is bounded. Therefore [GeGé] gives the result.

Lemma 3.17. We have $\Delta_{\mathcal{G}^{\mathrm{c}}} \in \mathcal{C}^{2}\left(\mathcal{A}_{\mathcal{G}^{\mathrm{c}}}\right)$ and

$$
\left[\left[\Delta_{\mathcal{G}^{\mathrm{c}}}, \mathrm{i} \mathcal{A}_{\mathcal{G}^{\mathrm{c}}}\right]_{\circ}, \mathrm{i} \mathcal{A}_{\mathcal{G}^{\mathrm{c}}}\right]_{\mathrm{o}} \simeq\left[\left[\Delta_{\mathcal{G}^{\mathrm{c}}}^{\mathrm{le}}, \mathrm{i} \mathcal{A}_{\mathcal{G}^{\mathrm{c}}}^{\mathrm{le}}\right]_{\circ}, \mathrm{i} \mathcal{A}_{\mathcal{G}^{\mathrm{c}}}^{\mathrm{le}}\right]_{\circ} \oplus 0
$$

Proof. Recalling (3.21) and Lemma 2.1, the result follows from noticing that $w^{\mathrm{c}}\left(\Delta_{\mathcal{G}^{\mathrm{c}}}^{\mathrm{le}}\right)$ is in $\mathcal{C}^{1}\left(\mathcal{A}_{\mathcal{G}^{\mathrm{c}}}^{\mathrm{le}}\right)$ as product of bounded elements of $\mathcal{C}^{1}\left(\mathcal{A}_{\mathcal{G}^{\mathrm{c}}}^{\mathrm{le}}\right)$.

Concerning the Mourre estimate, we prove the following result:

Proposition 3.18. We have $\Delta_{\mathcal{G}^{c}} \in \mathcal{C}^{2}\left(\mathcal{A}_{\mathcal{G}^{\mathrm{c}}}\right)$. Given a compact interval $\mathcal{I} \subset$ $\left(\frac{\alpha}{m_{2}}, \frac{\beta}{m_{2}}\right)$, there are $c>0$, a compact operator $K$ such that

$$
E_{\mathcal{I}}\left(\Delta_{\mathcal{G}^{\mathrm{c}}}\right)\left[\Delta_{\mathcal{G}^{\mathrm{c}}}, \mathrm{i} \mathcal{A}_{\mathcal{G}^{\mathrm{c}}}\right]_{\circ} E_{\mathcal{I}}\left(\Delta_{\mathcal{G}^{\mathrm{c}}}\right) \geq c E_{\mathcal{I}}\left(\Delta_{\mathcal{G}^{\mathrm{c}}}\right)+K
$$

in the form sense.

Proof. The Lemma 3.17 provides that $\Delta_{\mathcal{G}^{\mathrm{c}}} \in \mathcal{C}^{2}\left(\mathcal{A}_{\mathcal{G}^{\mathrm{c}}}\right)$. On $\mathcal{H}^{\text {he }}, E_{\mathcal{I}}\left(\Delta_{\mathcal{G}^{\mathrm{c}}}^{\text {he }}\right)$ is compact since $\Delta_{\mathcal{G}^{\mathrm{c}}}^{\text {he }}$ is with compact resolvent and $\mathcal{I}$ is with compact support. With respect to $(3.17)$, we have $E_{\mathcal{I}}\left(\Delta_{\mathcal{G}^{\mathrm{c}}}\right)=E_{\mathcal{I}}\left(\Delta_{\mathcal{G}^{\mathrm{c}}}^{\mathrm{le}}\right) \oplus E_{\mathcal{I}}\left(\Delta_{\mathcal{G}^{\mathrm{c}}}^{\mathrm{he}}\right)$ and

$$
\begin{aligned}
E_{\mathcal{I}}\left(\Delta_{\mathcal{G}^{\mathrm{c}}}\right)\left[\Delta_{\mathcal{G}^{\mathrm{c}}}, \mathrm{i} \mathcal{A}_{\mathcal{G}^{\mathrm{c}}}\right]_{\circ} E_{\mathcal{I}}\left(\Delta_{\mathcal{G}^{\mathrm{c}}}\right) & =E_{\mathcal{I}}\left(\Delta_{\mathcal{G}^{\mathrm{c}}}^{\mathrm{le}}\right)\left[\Delta_{\mathcal{G}^{\mathrm{c}}}^{\mathrm{le}}, \mathrm{i} \mathcal{A}_{\mathcal{G}^{\mathrm{c}}}^{\mathrm{le}}\right]_{\circ} E_{\mathcal{I}}\left(\Delta_{\mathcal{G}^{\mathrm{c}}}^{\mathrm{le}}\right) \oplus 0 \\
& \geq c E_{\mathcal{I}}\left(\Delta_{\mathcal{G}^{\mathrm{c}}}^{\mathrm{le}}\right) \oplus 0 \geq c E_{\mathcal{I}}\left(\Delta_{\mathcal{G}^{\mathrm{c}}}\right)+K,
\end{aligned}
$$

in the form sense, where $K$ is a compact operator and

$$
c:=\frac{m_{2}}{2} \inf _{x \in I}\left(x-\frac{\alpha}{m_{2}}\right)\left(\frac{\beta}{m_{2}}-x\right)>0 .
$$

This concludes the proof.

To lighten the text we did not expand more consequences of the Mourre theory in this case and refer to Theorem 4.3 for them.

3.5. The compact part. We define the conjugate operator on $\ell^{2}(\mathcal{V})=\ell^{2}\left(\mathcal{V}^{\mathrm{f}}\right) \oplus$ $\ell^{2}\left(\mathcal{V}^{0}\right) \oplus \ell^{2}\left(\mathcal{V}^{\mathrm{c}}\right)$ as

$$
\begin{aligned}
\ell^{2}(\mathcal{V}) & =\ell^{2}\left(\mathcal{V}^{\mathrm{f}}\right) \oplus \ell^{2}\left(\mathcal{V}^{0}\right) \oplus \ell^{2}\left(\mathcal{V}^{\mathrm{c}}\right) \\
\mathcal{A} & :=\mathcal{A}_{\mathcal{G}^{\mathrm{f}}} \oplus 0 \oplus \mathcal{A}_{\mathcal{G}^{\mathrm{c}}}
\end{aligned}
$$

Since $\mathcal{V}^{0}$ is finite, we have a finite rank perturbation and we conclude that $\mathcal{A}$ is self-adjoint and essentially self-adjoint on $\mathcal{C}_{c}(\mathcal{V})$.

Lemma 3.19. We have $\Delta_{\mathcal{G}} \in \mathcal{C}^{2}(\mathcal{A})$.

Proof. We have $\left(\Delta_{\mathcal{G}}-\Delta_{\mathcal{G}^{\mathrm{f}}} \oplus 0 \oplus \Delta_{\mathcal{G}^{\mathrm{c}}}\right)$ that are with finite support. Hence it belongs to $\mathcal{C}^{2}(\mathcal{A})$ by Lemma 2.1. Next recalling Lemma 3.11 and Lemma 3.17 we obtain the result. 
3.6. The whole graph. In this section, we give the Mourre estimate in the whole graph.

Proposition 3.20. We have $\Delta_{\mathcal{G}} \in \mathcal{C}^{2}(\mathcal{A})$. Given a compact interval $\mathcal{I} \subset\left(\frac{\alpha}{m_{2}}, \frac{\beta}{m_{2}}\right)$ Moreover, there are $c>0$, a compact operator $K$ such that

$$
E_{\mathcal{I}}\left(\Delta_{\mathcal{G}}\right)\left[\Delta_{\mathcal{G}}, \mathrm{i} \mathcal{A}\right]_{\circ} E_{\mathcal{I}}\left(\Delta_{\mathcal{G}}\right) \geq c E_{\mathcal{I}}\left(\Delta_{\mathcal{G}}\right)+K
$$

Proof. First $\Delta_{\mathcal{G}} \in \mathcal{C}^{2}(\mathcal{A})$ by Lemma 3.19 . Then by collecting (3.25) and (3.14), we obtain

$$
\begin{aligned}
E_{\mathcal{I}}\left(\Delta_{\mathcal{G}^{\mathrm{f}}} \oplus 0 \oplus \Delta_{\mathcal{G}^{\mathrm{c}}}\right)\left[\Delta_{\mathcal{G}^{\mathrm{f}}} \oplus 0 \oplus \Delta_{\mathcal{G}^{\mathrm{c}}}, \mathrm{i} \mathcal{A}\right]_{\circ} E_{\mathcal{I}}\left(\Delta_{\mathcal{G}^{\mathrm{f}}} \oplus 0 \oplus \Delta_{\mathcal{G}^{\mathrm{c}}}\right) \\
\\
\geq c E_{\mathcal{I}}\left(\Delta_{\mathcal{G}^{\mathrm{f}}} \oplus 0 \oplus \Delta_{\mathcal{G}^{\mathrm{c}}}\right)+K .
\end{aligned}
$$

Since the operators $\Delta_{\mathcal{G}}$ and $\Delta_{\mathcal{G}^{\mathrm{f}}} \oplus 0 \oplus \Delta_{\mathcal{G}^{\mathrm{c}}}$ are in $\mathcal{C}_{u}^{1}(\mathcal{A})$ (as in $\mathcal{C}^{2}(A)$, see [AmBoGe]), [AmBoGe, Theorem 7.2.9] implies (3.26).

\section{The PERTURBed MODEL}

In this section, we perturb the metrics of the previous case which will be small to infinity. We obtain similar results however the proof is more involved because we rely on the optimal class $\mathcal{C}^{1,1}(\mathcal{A})$ of the Mourre theory.

4.1. Perturbation of the metric. Let $\mathcal{G}_{\varepsilon, \mu}:=\left(\mathcal{V}, \mathcal{E}_{\varepsilon}, m_{\mu}\right)$ where

$$
m_{\mu}(x):=(1+\mu(x)) m(x) \text { and } \mathcal{E}_{\varepsilon}(x, y):=(1+\varepsilon(x, y)) \mathcal{E}(x, y),
$$

where $\mu>-1, \varepsilon>-1$, and

$$
\mu(x) \rightarrow 0 \text { if }|x| \rightarrow \infty \text { and } \varepsilon(x, y) \rightarrow 0 \text { if }|x|,|y| \rightarrow \infty .
$$

We set

$$
m_{\mu}^{*}:=\left.m_{\mu}\right|_{\mathcal{V}^{*}}, \quad \mathcal{E}_{\varepsilon}^{*}:=\left.\mathcal{E}_{\varepsilon}\right|_{\mathcal{V}^{*} \times \mathcal{V}^{*}},
$$

$\mu^{*}:=\left.\mu^{*}\right|_{\mathcal{V}^{*}}$, and $\varepsilon^{*}:=\varepsilon_{\mathcal{V}^{*} \times \mathcal{V}^{*}}$, with $* \in\{\mathrm{c}, \mathrm{f}\}$.

To analyse the spectral properties of $\Delta_{\mathcal{G}_{\varepsilon, \mu}}$, we compare it to $\Delta_{\mathcal{G}}$. As they do not act in the same spaces, we rely on Proposition 3.1. and send $\Delta_{\mathcal{G}_{\varepsilon, \mu}}$ in $\ell^{2}(\mathcal{V}, m)$ with the help of the unitary transformation. Namely, supposing (4.1). Let

$$
\widetilde{\Delta}_{\mathcal{G}_{\varepsilon, \mu}}:=T_{m_{\mu} \rightarrow m} \Delta_{\mathcal{G}_{\varepsilon, \mu}} T_{m_{\mu} \rightarrow m}^{-1} .
$$

A straightforward calculus ensures:

Lemma 4.1. For all $f \in \mathcal{C}_{c}(\mathcal{V})$, we have

$$
\begin{aligned}
& \left(\widetilde{\Delta}_{\mathcal{G}_{\varepsilon, \mu}}-\Delta_{\mathcal{G}}\right) f(x):=\frac{1}{m(x)} \sum_{y \sim x}\left(\frac{\varepsilon(x, y)}{\sqrt{(1+\mu(x))(1+\mu(y))}}\right. \\
& (4.2) \\
& \left.-\frac{\mu(x)+\mu(y)+\mu(x) \mu(y)}{\sqrt{(1+\mu(x))(1+\mu(z))}(1+\sqrt{(1+\mu(x))(1+\mu(z))})}\right) \mathcal{E}(x, z)(f(x)-f(y)) \\
& -\frac{1}{m(x)} \sum_{z \sim x}(1+\varepsilon(x, z)) \mathcal{E}(x, z) \frac{\mu(z)-\mu(x)}{(1+\mu(x)) \sqrt{1+\mu(z)}(\sqrt{1+\mu(z)}+\sqrt{1+\mu(x)})} f(x) .
\end{aligned}
$$

Proposition 4.2. Let $V: \mathcal{V} \rightarrow \mathbb{R}$ be a function, obeying $V(x) \rightarrow 0$ if $|x| \rightarrow \infty$. We assume that (4.1) holds true, then $\widetilde{\Delta}_{\mathcal{G}_{\varepsilon, \mu}}-\Delta_{\mathcal{G}} \in \mathcal{K}\left(\ell^{2}(\mathcal{V}), m\right)$. In particular

(1) $\mathcal{D}\left(\Delta_{\mathcal{G}_{\varepsilon, \mu}}+V(\cdot)\right)=\mathcal{D}\left(T_{m_{\mu} \rightarrow m}^{-1} \Delta_{\mathcal{G}} T_{m_{\mu} \rightarrow m}\right)$,

(2) $\Delta_{\mathcal{G}_{\varepsilon, \mu}}+V(\cdot)$ is essentially self-adjoint on $\mathcal{C}_{c}(\mathcal{V})$,

(3) $\sigma_{\mathrm{ess}}\left(\Delta_{\mathcal{G}_{\varepsilon, \mu}}+V(\cdot)\right)=\sigma_{\mathrm{ess}}\left(\Delta_{\mathcal{G}}\right)$.

Proof. Use Propositions 4.4 and 4.9 and note that the contribution arising from $\mathcal{V}^{0}$ is a finite rank perturbation. 
4.2. Main result. The main result of this section is the following theorem:

Theorem 4.3. Let $\mathcal{G}_{\varepsilon, \mu}$ a graph satisfies a condition (4.1) and

$$
\mathcal{A}_{\mathcal{G}_{\varepsilon, \mu}}:=\mathcal{A}_{\mathcal{G}_{\varepsilon, \mu}^{\mathrm{f}}} \oplus 0 \oplus \mathcal{A}_{\mathcal{G}_{\varepsilon, \mu}^{\mathrm{c}}}
$$

be a self-adjoint operator, where $\mathcal{A}_{\mathcal{G}_{\varepsilon, \mu}^{*}}:=T_{m_{\mu^{*} \rightarrow m}}^{-1} \mathcal{A}_{\mathcal{G}^{*}} T_{m_{\mu^{*} \rightarrow m}}$ with $* \in\{\mathrm{f}, \mathrm{c}\}$. Let $V: \mathcal{V} \rightarrow \mathbb{R}$ be a function such that $V, \varepsilon$, and $\mu$ are radial on $\mathcal{V}^{\mathrm{c}}$ (see Definition 4.10).

We assume that:

$$
\begin{aligned}
& (H 1) \sup _{\left(x_{1}, x_{2}\right) \in \mathcal{V}^{*}}\left\langle x_{1}\right\rangle^{1+\epsilon}\left|V\left(x_{1}-1, x_{2}\right)-V\left(x_{1}, x_{2}\right)\right|<\infty, \\
& (H 2) \sup _{\left(x_{1}, x_{2}\right) \in \mathcal{V}^{*}}\left\langle x_{1}\right\rangle^{\epsilon+1}\left|\mu^{*}\left(x_{1}-1, x_{2}\right)-\mu^{*}\left(x_{1}, x_{2}\right)\right|<\infty, \\
& (H 3) \sup _{\left(x_{1}, x_{2}\right) \in \mathcal{V}^{*}}\left\langle x_{1}\right\rangle^{\epsilon+1}\left|\varepsilon^{*}\left(\left(x_{1}, x_{2}\right),\left(x_{1}+1, x_{2}\right)\right)-\varepsilon^{*}\left(\left(x_{1}-1, x_{2}\right),\left(x_{1}, x_{2}\right)\right)\right|<\infty,
\end{aligned}
$$

where $V(x) \rightarrow 0$ if $|x| \rightarrow \infty$. Then $\Delta_{\mathcal{G}_{\varepsilon, \mu}}+V(\cdot) \in \mathcal{C}^{1,1}\left(\mathcal{A}_{\mathcal{G}_{\varepsilon, \mu}}\right)$. Moreover, for all compact interval $I \subset\left(\frac{\alpha}{m_{2}}, \frac{\beta}{m_{2}}\right)$, with $\alpha, \beta$ are given in $(1.2)$, there are $c>0$ and a compact operator $K$ such that

$$
\begin{gathered}
E_{I}\left(\Delta_{\mathcal{G}_{\varepsilon, \mu}}+V(\cdot)\right)\left[\Delta_{\mathcal{G}_{\varepsilon, \mu}}+V(\cdot), \mathrm{i}_{\mathcal{G}_{\varepsilon, \mu}}\right]_{\circ} E_{I}\left(\Delta_{\mathcal{G}_{\varepsilon, \mu}}+V(\cdot)\right) \\
\geq c E_{I}\left(\Delta_{\mathcal{G}_{\varepsilon, \mu}}+V(\cdot)\right)+K .
\end{gathered}
$$

in the form sense. Set $\kappa\left(\Delta_{\mathcal{G}_{\varepsilon, \mu}}+V(\cdot)\right):=\sigma_{p}\left(\Delta_{\mathcal{G}_{\varepsilon, \mu}}+V(\cdot)\right) \cup\left\{\frac{\alpha}{m_{2}}, \frac{\beta}{m_{2}}\right\}$ where $\sigma_{p}$ denotes the pure point spectrum. Take $s>1 / 2$ and $[a, b] \subset \mathbb{R} \backslash \kappa\left(\Delta_{\mathcal{G}_{\varepsilon, \mu}}+V(\cdot)\right)$. We obtain:

(2) The eigenvalues of $\Delta_{\mathcal{G}_{\varepsilon, \mu}}+V(\cdot)$ distinct from $\alpha$ and $\beta$ are of finite multiplicity and can accumulate only toward $\alpha$ and $\beta$.

(3) The singular continuous spectrum of $\Delta_{\mathcal{G}_{\varepsilon, \mu}}+V(\cdot)$ is empty.

(4) The following limit exists and finite:

$$
\lim _{\rho \rightarrow 0} \sup _{\lambda \in[a, b]}\left\|\langle\Lambda\rangle^{-s}\left(\Delta_{\mathcal{G}_{\varepsilon, \mu}}+V(\cdot)-\lambda-\mathrm{i} \rho\right)^{-1}\langle\Lambda\rangle^{-s}\right\|<\infty
$$

(5) There exists $c>0$ such that for all $f \in \ell^{2}\left(\mathcal{V}, m_{\mu}\right)$, we have:

$$
\int_{\mathbb{R}}\left\|\langle\Lambda\rangle^{-s} e^{-\mathrm{i} t\left(\Delta_{\mathcal{G}_{\varepsilon, \mu}}+V(\cdot)\right)} E_{[a, b]}\left(\Delta_{\mathcal{G}_{\varepsilon, \mu}}+V(\cdot)\right) f\right\|^{2} d t \leq c\|f\|^{2},
$$

with $\Lambda:=\Lambda^{\mathrm{f}} \oplus 0 \oplus \Lambda^{\mathrm{c}}$.

Proof. First $\Delta_{\mathcal{G}_{\varepsilon, \mu}}+V(\cdot) \in \mathcal{C}^{1,1}\left(\mathcal{A}_{\mathcal{G}_{\varepsilon, \mu}}\right)$ because $\Delta_{\mathcal{G}_{\varepsilon, \mu}^{\mathrm{f}}} \oplus 0 \oplus \Delta_{\mathcal{G}_{\varepsilon, \mu}^{\mathrm{c}}} \in \mathcal{C}^{1,1}\left(\mathcal{A}_{\mathcal{G}_{\varepsilon, \mu}}\right)$ by the Lemma 4.8, the Lemma 4.14, the Lemma 4.13, the Lemma 4.7 and by Lemma 3.19. In particular, we have that the two operators are in $\mathcal{C}_{u}^{1}\left(\mathcal{A}_{\mathcal{G}_{\varepsilon, \mu}}\right)$, see $[\mathrm{AmBoGe}]$.

Then, using the Proposition 4.5 and the Proposition 4.11 we obtain

$$
\begin{aligned}
E_{I}\left(\Delta_{\mathcal{G}_{\varepsilon, \mu}^{\mathrm{f}}} \oplus 0 \oplus \Delta_{\mathcal{G}_{\varepsilon, \mu}^{\mathrm{c}}}+V(\cdot)\right)\left[\Delta_{\mathcal{G}_{\varepsilon, \mu}^{\mathrm{f}}} \oplus 0 \oplus \Delta_{\mathcal{G}_{\varepsilon, \mu}^{\mathrm{c}}}+V(\cdot), \mathrm{i} \mathcal{A}_{\mathcal{G}_{\varepsilon, \mu}}\right]_{\circ} \\
E_{I}\left(\Delta_{\mathcal{G}_{\varepsilon, \mu}^{\mathrm{f}}} \oplus 0 \oplus \Delta_{\mathcal{G}_{\varepsilon, \mu}^{\mathrm{c}}}+V(\cdot)\right) \\
\geq c E_{I}\left(\Delta_{\mathcal{G}_{\varepsilon, \mu}^{\mathrm{f}}} \oplus 0 \oplus \Delta_{\mathcal{G}_{\varepsilon, \mu}^{\mathrm{c}}}+V(\cdot)\right)+K .
\end{aligned}
$$

Since $\Delta_{\mathcal{G}_{\varepsilon, \mu}^{\mathrm{f}}} \oplus 0 \oplus \Delta_{\mathcal{G}_{\varepsilon, \mu}^{\mathrm{c}}} \in \mathcal{C}_{u}^{1}\left(\mathcal{A}_{\mathcal{G}_{\varepsilon, \mu}}\right), \Delta_{\mathcal{G}_{\varepsilon, \mu}^{\mathrm{f}}} \oplus 0 \oplus \Delta_{\mathcal{G}_{\varepsilon, \mu}^{\mathrm{c}}}-\Delta_{\mathcal{G}_{\varepsilon, \mu}} \in \mathcal{K}\left(\ell^{2}\left(\mathcal{V}, m_{\mu}\right)\right)$, and $V(\cdot) \in \mathcal{C}_{u}^{1}\left(\mathcal{A}_{\mathcal{G}_{\varepsilon, \mu}}\right)$ and by $[$ AmBoGe, Theorem 7.2.9], we obtain (4.3). By Lemma 4.7 and Lemma 4.13, $V(\cdot) \in \mathcal{C}^{1,1}\left(\mathcal{A}_{\mathcal{G}_{\varepsilon, \mu}}\right)$. And by using Proposition 4.4 and Proposition 4.9 , we have that $\left(\Delta_{\mathcal{G}_{\varepsilon, \mu}^{\mathrm{f}}} \oplus 0 \oplus \Delta_{\mathcal{G}_{\varepsilon, \mu}^{\mathrm{c}}}+\mathrm{i}\right)^{-1}-\left(\Delta_{\mathcal{G}_{\varepsilon, \mu}}+\mathrm{i}\right)^{-1} \in \mathcal{K}\left(\ell^{2}\left(\mathcal{V}, \mathrm{m}_{\mu}\right)\right)$. Finally, we turn to points $(4)$. It is enough to obtain them with $s \in(1 / 2,1)$. We apply [AmBoGe, Proposition 7.5.6] and obtain

$$
\lim _{\rho \rightarrow 0}\left\|\langle\mathcal{A}\rangle^{-s}\left(\Delta_{\mathcal{G}_{\varepsilon, \mu}}+V(\cdot)-\lambda-\mathrm{i} \rho\right)^{-1}\langle\mathcal{A}\rangle^{-\mathrm{s}}\right\|
$$


exists and finite. Using Propositions 4.6 b) and 4.12 b)

$$
\left\|\left\langle\mathcal{A}_{\mathcal{G}_{\varepsilon, \mu}}\right\rangle f\right\| \leq a\|\langle\Lambda\rangle f\|,
$$

for all $f \in \mathcal{D}(\Lambda)$. By Riesz-Thorin interpolation, there is $a_{s}>0$ such that

$$
\left\|\left\langle\mathcal{A}_{\mathcal{G}_{\varepsilon, \mu}}\right\rangle^{s} f\right\| \leq a_{s}\left\|\langle\Lambda\rangle^{s} f\right\|
$$

for all $f \in \mathcal{D}\left(\Lambda^{s}\right)$. We conclude that $\lim _{\rho \rightarrow 0}\left\|\langle\Lambda\rangle^{-s}\left(\Delta_{\mathcal{G}_{\varepsilon, \mu}}+V(\cdot)-\lambda-\mathrm{i} \rho\right)^{-1}\langle\Lambda\rangle^{-\mathrm{s}}\right\|$ exists and finite. The point (5) is an immediate consequence of (4).

4.3. The funnel side. We first deal with the question of the essential spectrum.

Proposition 4.4. Let $V^{\mathrm{f}}: \mathcal{V}^{\mathrm{f}} \rightarrow \mathbb{R}$ be a function obeying $V^{\mathrm{f}}(x) \rightarrow 0$ if $|x| \rightarrow \infty$. We assume that (4.1) holds true then $\widetilde{\Delta}_{\mathcal{G}_{\varepsilon, \mu}^{\mathrm{f}}}-\Delta_{\mathcal{G}^{\mathrm{f}}} \in \mathcal{K}\left(\ell^{2}\left(\mathcal{V}^{\mathrm{f}}\right), m^{\mathrm{f}}\right)$, where $\widetilde{\Delta}_{\mathcal{G}_{\varepsilon, \mu}}:=$ $T_{m_{\mu} \rightarrow m} \Delta_{\mathcal{G}_{\varepsilon, \mu}} T_{m_{\mu} \rightarrow m}^{-1}$. In particular,

(1) $\mathcal{D}\left(\Delta_{\mathcal{G}_{\varepsilon, \mu}^{\mathrm{f}}}+V(\cdot)\right)=\mathcal{D}\left(T_{m_{\mu} \rightarrow m}^{-1} \Delta_{\mathcal{G}} T_{m_{\mu} \rightarrow m}\right)$,

(2) $\Delta_{\mathcal{G}_{\varepsilon, \mu}^{\mathrm{f}}}+V(\cdot)$ is essentially self-adjoint on $\mathcal{C}_{c}(\mathcal{V})$,

(3) $\sigma_{\mathrm{ess}}\left(\Delta_{\mathcal{G}_{\varepsilon, \mu}^{\mathrm{f}}}+V(\cdot)\right)=\sigma_{\mathrm{ess}}\left(\Delta_{\mathcal{G}}\right)$.

Proof. We shall show that $\widetilde{\Delta}_{\mathcal{G}_{\varepsilon, \mu}^{\mathrm{f}}}-\Delta_{\mathcal{G}^{\mathrm{f}}} \in \mathcal{K}\left(\ell^{2}\left(\mathcal{V}^{\mathrm{f}}, m^{\mathrm{f}}\right)\right)$, as in $(4.2)$. Let $f \in \mathcal{C}_{c}(\mathcal{V})$,

$$
\begin{aligned}
& \left|\left\langle f,\left(\widetilde{\Delta}_{\mathcal{G}_{\varepsilon, \mu}^{\mathrm{f}}}-\Delta_{\mathcal{G}^{\mathrm{f}}}\right) f\right\rangle_{\ell^{2}\left(\mathcal{V}^{\mathrm{f}}, m^{\mathrm{f}}\right)}\right|=\left|\sum_{x \in \mathcal{V}^{\mathrm{f}}} m^{\mathrm{f}}(x)\left(\left(\widetilde{\Delta}_{\mathcal{G}_{\varepsilon, \mu}^{\mathrm{f}}}-\Delta_{\mathcal{G}^{\mathrm{f}}}\right) f\right)(x) \overline{f(x)}\right| \\
& \leq \mid \sum_{x \in \mathcal{V}^{\mathrm{f}}} m^{\mathrm{f}}(x) \frac{1}{m^{\mathrm{f}}(x)} \sum_{z \sim x} \frac{\varepsilon^{\mathrm{f}}(x, z)}{\sqrt{\left(1+\mu^{\mathrm{f}}(x)\right)\left(1+\mu^{\mathrm{f}}(z)\right)}} \mathcal{E}^{\mathrm{f}}(x, z) \\
& \quad \times(f(x)-f(z)) \overline{f(x)} \mid \\
& +\mid \sum_{x \in \mathcal{V}^{\mathrm{f}}} m^{\mathrm{f}}(x) \frac{1}{m^{\mathrm{f}}(x)} \sum_{z \sim x} \frac{1-\sqrt{\left(1+\mu^{\mathrm{f}}(x)\right)\left(1+\mu^{\mathrm{f}}(z)\right)}}{\sqrt{\left(1+\mu^{\mathrm{f}}(x)\right)\left(1+\mu^{\mathrm{f}}(z)\right)}} \mathcal{E}^{\mathrm{f}}(x, z) \\
& \quad \times(f(x)-f(z)) \overline{f(x)}|+|\left\langle f, W^{\mathrm{f}}(\cdot) f\right\rangle \mid \\
& \leq 2\left\langle f,\left(\operatorname{deg}_{1}(\cdot)+\operatorname{deg}_{2}(\cdot)+\left|W^{\mathrm{f}}(\cdot)\right|\right) f\right\rangle
\end{aligned}
$$

with

$$
\operatorname{deg}_{1}(x):=\frac{1}{m^{\mathrm{f}}(x)} \sum_{z \in \mathcal{V}^{\mathrm{f}}} \frac{\varepsilon^{\mathrm{f}}(x, z)}{\sqrt{\left(1+\mu^{\mathrm{f}}(x)\right)\left(1+\mu^{\mathrm{f}}(z)\right)}} \mathcal{E}^{\mathrm{f}}(x, z)
$$

and

$$
\operatorname{deg}_{2}(x):=\frac{1}{m^{\mathrm{f}}(x)} \sum_{z \in \mathcal{V}^{\mathrm{f}}}\left|\frac{1-\sqrt{\left(1+\mu^{\mathrm{f}}(x)\right)\left(1+\mu^{\mathrm{f}}(z)\right)}}{\sqrt{\left(1+\mu^{\mathrm{f}}(x)\right)\left(1+\mu^{\mathrm{f}}(z)\right)}}\right| \mathcal{E}^{\mathrm{f}}(x, z),
$$

for all $x=\left(x_{1}, x_{2}\right) \in \mathcal{V}^{\mathrm{f}}$. We have

$$
\begin{aligned}
\left|\operatorname{deg}_{1}(x)\right| & =\left|\frac{1}{m^{\mathrm{f}}(x)} \sum_{z \in \mathcal{V}^{\mathrm{f}}} \frac{\varepsilon^{\mathrm{f}}(x, z)}{\sqrt{\left(1+\mu^{\mathrm{f}}(x)\right)\left(1+\mu^{\mathrm{f}}(z)\right)}} \mathcal{E}^{\mathrm{f}}(x, z)\right| \\
& \leq \sup _{z \sim x}\left|\frac{\varepsilon^{\mathrm{f}}(x, z)}{\sqrt{\left(1+\mu^{\mathrm{f}}(x)\right)\left(1+\mu^{\mathrm{f}}(z)\right)}}\right| \operatorname{deg}_{\mathcal{G}^{\mathrm{f}}}(x) .
\end{aligned}
$$

Since $\mathcal{V}_{2}$ is a finite set and for all $x_{2} \in \mathcal{V}_{2}, \frac{\varepsilon^{\mathrm{f}}\left(\left(x_{1}, x_{2}\right),\left(z_{1}, z_{2}\right)\right)}{\sqrt{\left(1+\mu^{\mathrm{f}}\left(x_{1}, x_{2}\right)\right)\left(1+\mu^{\mathrm{f}}\left(z_{1}, z_{2}\right)\right)}} \rightarrow 0$ when $x_{1}, z_{1} \rightarrow \infty$ and $\operatorname{since} \operatorname{deg}_{\mathcal{G}^{\mathrm{f}}}(\cdot)$ is bounded then $\operatorname{deg}_{1}(\cdot)$ is compact. In the same way, using that $\forall x_{2}, z_{2} \in \mathcal{V}_{2}, \frac{1-\sqrt{\left(1+\mu^{\mathrm{f}}\left(x_{1}, x_{2}\right)\right)\left(1+\mu^{\mathrm{f}}\left(z_{1}, z_{2}\right)\right)}}{\sqrt{\left(1+\mu^{\mathrm{f}}\left(x_{1}, x_{2}\right)\left(1+\mu^{\mathrm{f}}\left(z_{1}, z_{2}\right)\right)\right.}} \rightarrow 0$ if $x_{1}, z_{1} \rightarrow \infty$, we obtain the compactness of $\operatorname{deg}_{2}(\cdot)$. 
Now, we will show that $W^{\mathrm{f}} \in \mathcal{K}\left(\ell^{2}\left(\mathcal{V}^{\mathrm{f}}, m^{\mathrm{f}}\right)\right)$. For all $x \in \mathcal{V}^{\mathrm{f}}$, we have

$$
\begin{aligned}
\left|W^{\mathrm{f}}(x)\right|= & \mid \frac{1}{m^{\mathrm{f}}(x)} \sum_{z \sim x}\left(1+\varepsilon^{\mathrm{f}}(x, z)\right) \mathcal{E}^{\mathrm{f}}(x, z) \\
& \times\left(\frac{\mu^{\mathrm{f}}(z)-\mu^{\mathrm{f}}(x)}{\left(1+\mu^{\mathrm{f}}(x)\right) \sqrt{1+\mu^{\mathrm{f}}(z)}\left(\sqrt{1+\mu^{\mathrm{f}}(z)}+\sqrt{1+\mu^{\mathrm{f}}(x)}\right)}\right) \mid \\
\leq & \sup _{z \sim x}\left|\left(1+\varepsilon^{\mathrm{f}}(x, z)\right)\left(\frac{\mu^{\mathrm{f}}(z)-\mu^{\mathrm{f}}(x)}{\left(1+\mu^{\mathrm{f}}(x)\right) \sqrt{1+\mu^{\mathrm{f}}(z)}\left(\sqrt{1+\mu^{\mathrm{f}}(z)}+\sqrt{1+\mu^{\mathrm{f}}(x)}\right)}\right)\right| \\
& \times \operatorname{deg}_{\mathcal{G}^{\mathrm{f}}}(x) .
\end{aligned}
$$

Since $\mathcal{V}_{2}$ is a finite set and $\left(1+\varepsilon^{\mathrm{f}}(x, z)\right)\left(\mu^{\mathrm{f}}(z)-\mu^{\mathrm{f}}(x)\right) \rightarrow 0$ when $|x|,|z| \rightarrow \infty$, $\operatorname{deg}_{\mathcal{G}^{\mathrm{f}}}(\cdot)$ is bounded and since $V^{\mathrm{f}}(\cdot)$ is a compact perturbation, we conclude that $\widetilde{\Delta}_{\mathcal{G}_{\varepsilon, \mu}^{\mathrm{f}}}-\Delta_{\mathcal{G}^{\mathrm{f}}}$ is compact. The points (1) and (2) follow from Theorem [ReSi, Theorem XIII.14] and (3) from the Weyl's Theorem.

We turn to the Mourre estimate.

Proposition 4.5. Let $V^{\mathrm{f}}: \mathcal{V}^{\mathrm{f}} \rightarrow \mathbb{R}$ be a function. We assume that $(H 1)$, (H2), and (H3) hold true, where $\varepsilon^{\mathrm{f}}(x, z) \rightarrow 0$ if $|x|,|z| \rightarrow \infty, \mu^{\mathrm{f}}(x) \rightarrow 0$ if $|x| \rightarrow \infty$ and $V^{\mathrm{f}}(x) \rightarrow 0$ if $|x| \rightarrow \infty$. Then $\Delta_{\mathcal{G}_{\varepsilon, \mu}^{\mathrm{f}}}+V^{\mathrm{f}}(\cdot) \in \mathcal{C}^{1.1}\left(\mathcal{A}_{\mathcal{G}_{\varepsilon, \mu}^{\mathrm{f}}}\right)$. Moreover, for all compact interval $I \subset\left(\frac{\alpha}{m_{2}}, \frac{\beta}{m_{2}}\right)$, there are $c>0$, a compact operator $K$ such that

$$
\begin{aligned}
E_{I}\left(\Delta_{\mathcal{G}_{\varepsilon, \mu}^{\mathrm{f}}}+V^{\mathrm{f}}(\cdot)\right)\left[\Delta_{\mathcal{G}_{\varepsilon, \mu}^{\mathrm{f}}}+V^{\mathrm{f}}(\cdot), \mathrm{i} \mathcal{A}_{\mathcal{G}_{\varepsilon, \mu}^{\mathrm{f}}}\right]_{\circ} E_{I}\left(\Delta_{\mathcal{G}_{\varepsilon, \mu}^{\mathrm{f}}}+V^{\mathrm{f}}(\cdot)\right) \\
\geq c E_{I}\left(\Delta_{\mathcal{G}_{\varepsilon, \mu}^{\mathrm{f}}}+V^{\mathrm{f}}(\cdot)\right)+K,
\end{aligned}
$$

in the form sense.

Proof. The Proposition 4.8 and Lemma 4.7 give that $\Delta_{\mathcal{G}_{\varepsilon, \mu}^{\mathrm{f}}}+V^{\mathrm{f}}(\cdot) \in \mathcal{C}^{1,1}\left(\mathcal{A}_{\mathcal{G}_{\varepsilon, \mu}^{\mathrm{f}}, \mu}\right)$. Since $\widetilde{\Delta}_{\mathcal{G}_{\varepsilon, \mu}^{\mathrm{f}}}-\Delta_{\mathcal{G}^{\mathrm{f}}}$ is a compact operator by Proposition 4.4 , thanks to $(3.14)$ and by $[$ AmBoGe, Theorem 7.2.9], we obtain (4.4).

We start with a technical lemma so as to apply [AmBoGe, Proposition 7.5.7].

Proposition 4.6. Let $\Lambda^{\mathrm{f}}:=(Q+1 / 2) \otimes \mathbf{1}_{\mathcal{V}^{\mathrm{f}}}$. It satisfies the following assertions:

(1) $e^{\mathrm{i} \Lambda^{\mathrm{f}} t} \mathcal{D}\left(\Delta_{\mathcal{G}_{\varepsilon, \mu}^{\mathrm{f}}}\right) \subset \mathcal{D}\left(\Delta_{\mathcal{G}_{\varepsilon, \mu}^{\mathrm{f}}}\right)$ and there exists a finite constant $\mathrm{c}$, such that

$$
\left\|e^{\mathrm{i} \Lambda^{\mathrm{f}} t}\right\|_{\mathcal{B}\left(\mathcal{D}\left(\Delta_{\mathcal{G}_{\varepsilon}^{\mathrm{f}}, \mu}\right)\right)} \leq \mathrm{c}, \quad \text { for all } \mathrm{t} \in \mathbb{R} .
$$

(2) $\mathcal{D}\left(\Lambda^{\mathrm{f}}\right) \subset \mathcal{D}\left(\mathcal{A}_{\mathcal{G}_{\varepsilon, \mu}^{\mathrm{f}}}\right)$.

(3) $\left(\Lambda^{\mathrm{f}}\right)^{-2}\left(\mathcal{A}_{\mathcal{G}_{\varepsilon, \mu}^{\mathrm{f}}}\right)^{2}$ extends to a continuous operator in $\mathcal{D}\left(\Delta_{\mathcal{G}_{\varepsilon, \mu}^{\mathrm{f}}}\right)$.

Note that $\Delta_{\mathcal{G}_{\varepsilon, \mu}^{\mathrm{f}}}$ is bounded then $\mathcal{D}\left(\Delta_{\mathcal{G}_{\varepsilon, \mu}^{\mathrm{f}}}\right)=\ell^{2}\left(\mathcal{V}^{\mathrm{f}}, m_{\mu}^{\mathrm{f}}\right)$.

Proof. With the help of the unitary transformation $T_{m_{\mu} \rightarrow m}$, it is enough to prove the result with $\varepsilon=0$ and $\mu=0$.

(1) Since $\Delta_{\mathcal{G}^{\text {f }}}$ is bounded it is verified by a functional calculus.

(2) Let $f \in \mathcal{C}_{c}\left(\mathcal{V}^{\mathrm{f}}\right)$,

$$
\begin{aligned}
& \left\|\mathcal{A}_{\mathcal{G}^{\mathrm{f}}} f\right\|_{\ell^{2}\left(\mathcal{V}^{\mathrm{f}}, m^{\mathrm{f}}\right)}^{2} \\
& =\sum_{x \in \mathcal{V}^{\mathrm{f}}} m^{\mathrm{f}}(x)\left|\frac{\mathrm{i}}{2}\left(e^{1 / 2}(Q-1 / 2) U \otimes \mathbf{1}_{\mathcal{V}_{2}^{\mathrm{f}}}-e^{-1 / 2}(Q+1 / 2) U^{*} \otimes \mathbf{1}_{\mathcal{V}_{2}^{\mathrm{f}}}\right) f(x)\right|^{2} \\
& \leq c \sum_{x \in \mathcal{V}^{\mathrm{f}}} m^{\mathrm{f}}(x)\left|(Q+1 / 2) \otimes \mathbf{1}_{\mathcal{V}_{2}^{\mathrm{f}}} f(x)\right|^{2} \leq c\left\|\Lambda^{\mathrm{f}} f\right\|_{\ell^{2}\left(\mathcal{V}^{\mathrm{f}}, m^{\mathrm{f}}\right)}^{2} .
\end{aligned}
$$

Since $\Lambda^{\mathrm{f}}$ is essentially self-adjoint, the result follows. 
(3) For all $f \in \mathcal{C}_{c}\left(\mathcal{V}^{\mathrm{f}}\right)$, and by using the relations of Subsection 3.5, we have

$$
\begin{aligned}
& \mathcal{A}_{m_{1}}^{2} f(n)=\frac{1}{4}\left(2 n^{2}+1 / 2\right) f(n)-\frac{1}{4} e(n-1 / 2)(n-3 / 2) f(n-2) \\
& -\frac{1}{4} e^{-1}(n+1 / 2)(n+3 / 2) f(n+2) .
\end{aligned}
$$

Then for all $f \in \mathcal{C}_{c}\left(\mathcal{V}^{\mathrm{f}}\right)$.

$$
\begin{aligned}
& \left\|\left(\Lambda^{\mathrm{f}}\right)^{-2}\left(\mathcal{A}_{\mathcal{G}^{\mathrm{f}}}\right)^{2} f\right\|^{2}= \\
& \quad \sum_{\left(x_{1}, x_{2}\right) \in \mathcal{V}^{\mathrm{f}}} m^{\mathrm{f}}\left(x_{1}, x_{2}\right) \mid \frac{1}{4}\left(\left((Q+1 / 2)^{-2}\left(2 Q^{2}+1 / 2\right)\right) \otimes \mathbf{1}_{\mathcal{V}_{2}^{\mathrm{f}}}\right) f\left(x_{1}, x_{2}\right) \\
& \quad-\frac{1}{4} e\left(\left((Q+1 / 2)^{-2}(Q-1 / 2)(Q-3 / 2)\right) \otimes \mathbf{1}_{\mathcal{V}_{2}^{\mathrm{f}}}\right) f\left(x_{1}-2, x_{2}\right) \\
& \quad-\left.\frac{1}{4} e^{-1}\left(\left((Q+1 / 2)^{-2}(Q+1 / 2)(Q+3 / 2)\right) \otimes \mathbf{1}_{\mathcal{V}_{2}^{\mathrm{f}}}\right) f\left(x_{1}+2, x_{2}\right)\right|^{2} .
\end{aligned}
$$

Then, there exists $C>0$ such that for all $f \in \mathcal{C}_{c}\left(\mathcal{V}^{\mathrm{f}}\right),\left\|\left(\Lambda^{\mathrm{f}}\right)^{-2}\left(\mathcal{A}_{\mathcal{G}^{\mathrm{f}}}\right)^{2} f\right\|^{2} \leq C\|f\|^{2}$. By density, we find the result.

The proof of Proposition 4.8 will be long and technical. For the sake of the reader, we have separated the treatment of the potential $V^{\mathrm{f}}$ to present the technical steps.

Lemma 4.7. Let $V^{\mathrm{f}}: \mathcal{V}^{\mathrm{f}} \rightarrow \mathbb{R}$ be a function. We assume that $(H 1)$ holds true, then $V^{\mathrm{f}}(\cdot) \in \mathcal{C}^{1}\left(\mathcal{A}_{\mathcal{G}_{\varepsilon, \mu}^{\mathrm{f}}}\right)$ and $\left[V^{\mathrm{f}}(\cdot), \mathcal{A}_{\mathcal{G}_{\varepsilon, \mu}^{\mathrm{f}}}\right]_{\circ} \in \mathcal{C}^{0,1}\left(\mathcal{A}_{\mathcal{G}_{\varepsilon, \mu}^{\mathrm{f}}}\right)$. In particular, $V^{\mathrm{f}}(\cdot) \in$ $\mathcal{C}^{1,1}\left(\mathcal{A}_{\mathcal{G}_{\varepsilon, \mu}^{\mathrm{f}}}\right)$.

Proof. First, recalling $\left[V^{\mathrm{f}}(\cdot), \mathrm{i} \mathcal{A}_{\mathcal{G}_{\varepsilon, \mu}^{\mathrm{f}}}\right]_{\circ}=T_{m_{\mu^{*} \rightarrow m}}^{-1}\left[V^{\mathrm{f}}(\cdot), \mathrm{i} \mathcal{A}_{\mathcal{G}^{\mathrm{f}}}\right]_{\circ} T_{m_{\mu^{*}} \rightarrow m}$, it is enough to deal with $\epsilon=\mu=0$. Next, we recall that

$$
\left[V^{\mathrm{f}}(\cdot), \mathrm{i} \mathcal{A}_{\mathcal{G}^{\mathrm{f}}}\right]_{\circ}=\frac{e^{-1 / 2}}{2}\left(Q+\frac{1}{2}\right)\left[V^{\mathrm{f}}, U^{*}\right] \otimes \mathbf{1}_{\mathcal{V}_{2}^{\mathrm{f}}}+\frac{e^{1 / 2}}{2}\left(\frac{1}{2}-Q\right)\left[V^{\mathrm{f}}, U\right] \otimes \mathbf{1}_{\mathcal{V}_{2}^{\mathrm{f}}} .
$$

By using (H1) at the last step, there is $C$ such that, for all $f \in \mathcal{C}_{c}(\mathcal{V})$,

$$
\begin{aligned}
&\left\|\langle Q+1 / 2\rangle^{\epsilon} \otimes \mathbf{1}_{\mathcal{V}_{2}^{\mathrm{f}}}\left[V^{\mathrm{f}}(\cdot), \mathrm{i} \mathcal{A}_{\mathcal{G}^{\mathrm{f}}}\right]_{\circ} f\right\| \\
& \leq \frac{e^{-1 / 2}}{2}\left\|\left(\langle Q+1 / 2\rangle^{\epsilon}(Q+1 / 2)\left[V^{\mathrm{f}}, U^{*}\right]\right) \otimes \mathbf{1}_{\mathcal{V}_{2}^{\mathrm{f}}} f\right\| \\
&+\frac{e^{1 / 2}}{2}\left\|\left(\langle Q+1 / 2\rangle^{\epsilon}(Q+1 / 2)\left[V^{\mathrm{f}}, U\right]\right) \otimes \mathbf{1}_{\mathcal{V}_{2}^{\mathrm{f}}} f\right\| \\
& \leq \frac{e^{-1 / 2}}{2}\left\|\langle\Lambda\rangle^{\epsilon+1}\left(\left[V^{\mathrm{f}}, U^{*}\right] \otimes \mathbf{1}_{\mathcal{V}_{2}^{\mathrm{f}}}\right) f\right\|+\frac{e^{1 / 2}}{2}\left\|\langle\Lambda\rangle^{\epsilon+1}\left(\left[V^{\mathrm{f}}, U\right] \otimes \mathbf{1}_{\mathcal{V}_{2}^{\mathrm{f}}}\right) f\right\| \leq C\|f\| .
\end{aligned}
$$

Finally thanks to Proposition 4.6, we can apply [AmBoGe, Proposition 7.5.7] and the result follows.

We conclude this section with the most technical part.

Proposition 4.8. Assuming (H2) and (H3) hold true, we have $\Delta_{\mathcal{G}_{\varepsilon, \mu}^{\mathrm{f}}} \in \mathcal{C}^{1}\left(\mathcal{A}_{\mathcal{G}_{\varepsilon, \mu}^{\mathrm{f}}}\right)$. Moreover $\left[\Delta_{\mathcal{G}_{\varepsilon, \mu}^{\mathrm{f}}}, \mathcal{A}_{\mathcal{G}_{\varepsilon, \mu}^{\mathrm{f}}}\right]_{\circ} \in \mathcal{C}^{0,1}\left(\mathcal{A}_{\mathcal{G}_{\varepsilon, \mu}^{\mathrm{f}}}\right)$. In particular, $\Delta_{\mathcal{G}_{\varepsilon, \mu}^{\mathrm{f}}} \in \mathcal{C}^{1,1}\left(\mathcal{A}_{\mathcal{G}_{\varepsilon, \mu}^{\mathrm{f}}}\right)$.

Proof. We work in $\ell^{2}\left(\mathcal{V}^{\mathrm{f}}, m^{f}\right)$. First, using the computation below with $\epsilon=0$ and recalling that $\mathcal{A}_{\mathcal{G}^{\mathrm{f}}} \mathcal{C}_{c}\left(\mathcal{V}^{\mathrm{f}}\right) \subset \mathcal{C}_{c}\left(\mathcal{V}^{\mathrm{f}}\right)$, we get there is $c>0$ such that

$$
\left\|\left[\widetilde{\Delta}_{\mathcal{G}_{\varepsilon, \mu}^{\mathrm{f}},}, \mathcal{A}_{\mathcal{G}^{\mathrm{f}}}\right] f\right\|_{\ell^{2}\left(\mathcal{V}^{\mathrm{f}}, m^{\mathrm{f}}\right)} \leq c\|f\|_{\ell^{2}\left(\mathcal{V}^{\mathrm{f}}, m^{\mathrm{f}}\right)},
$$


for all $f \in \mathcal{C}_{c}\left(\mathcal{V}^{\mathrm{f}}\right)$. By density, we obtain that $\widetilde{\Delta}_{\mathcal{G}_{\varepsilon, \mu}^{\mathrm{f}}} \in \mathcal{C}^{1}\left(\mathcal{A}_{\mathcal{G}^{\mathrm{f}}}\right)$. Next take $\epsilon>0$ and $f \in \mathcal{C}_{c}\left(\mathcal{V}^{\mathrm{f}}\right)$. We aim at proving that $\left[\widetilde{\Delta}_{\mathcal{G}_{\varepsilon, \mu}^{\mathrm{f}}}, \mathcal{A}_{\mathcal{G}^{\mathrm{f}}}\right]_{\circ}$ is $\mathcal{C}^{0,1}\left(\mathcal{A}_{\mathcal{G}^{\mathrm{f}}}\right)$.

$$
\begin{aligned}
& \left\|\left\langle\Lambda^{\mathrm{f}}\right\rangle^{\epsilon}\left[\widetilde{\Delta}_{\mathcal{G}_{\varepsilon, \mu}^{\mathrm{f}}}, \mathcal{A}_{\mathcal{G}^{\mathrm{f}}}\right] f\right\|_{\ell^{2}\left(\mathcal{V}^{\mathrm{f}}, m^{\mathrm{f}}\right)}=\sum_{x \in \mathcal{V}^{\mathrm{f}}} m^{\mathrm{f}}(x)\left|\left\langle\Lambda^{\mathrm{f}}\right\rangle^{\epsilon}\left[\widetilde{\Delta}_{\mathcal{G}_{\varepsilon}^{\mathrm{f}}, \mu}, \mathcal{A}_{\mathcal{G}^{\mathrm{f}}}\right] f(x)\right|^{2} \\
& \leq \sum_{x \in \mathcal{V}^{\mathrm{f}}} m^{\mathrm{f}}(x) \mid \frac{\mathrm{i}}{2}\left(e^{1 / 2}\langle Q-1 / 2\rangle^{1+\epsilon} \otimes \mathbf{1}_{\mathcal{V}_{2}^{\mathrm{f}}}\right)
\end{aligned}
$$

$$
\begin{aligned}
& \times\left(\frac{1}{m^{\mathrm{f}}(x)} \sum_{z \sim x} \mathcal{E}^{\mathrm{f}}(x, z) \frac{1+\varepsilon^{\mathrm{f}}(x, z)}{\sqrt{\left(1+\mu^{\mathrm{f}}(x)\right)\left(1+\mu^{\mathrm{f}}(z)\right)}} f\left(x_{1}-1, x_{2}\right)\right. \\
& \left.-\frac{1}{m^{\mathrm{f}}\left(x_{1}-1, x_{2}\right)} \sum_{z \sim x} \mathcal{E}^{\mathrm{f}}\left(\left(x_{1}-1, x_{2}\right), z\right) f\left(z_{1}-1, z_{2}\right) \times \frac{1+\varepsilon^{\mathrm{f}}\left(\left(x_{1}-1, x_{2}\right), z\right)}{\sqrt{\left(1+\mu^{\mathrm{f}}\left(x_{1}-1, x_{2}\right)\left(1+\mu^{\mathrm{f}}(z)\right)\right.}}\right)\left.\right|^{2} \\
& +\sum_{x \in \mathcal{V}^{\mathrm{f}}} m^{\mathrm{f}}(x) \mid \frac{\mathrm{i}}{2}\left(e^{1 / 2}\langle Q-1 / 2\rangle^{1+\epsilon} \otimes \mathbf{1}_{\mathcal{V}_{2}^{\mathrm{f}}}\right) \\
& \times\left(\frac{1}{m^{\mathrm{f}}\left(x_{1}-1, x_{2}\right)} \sum_{z \sim x} \mathcal{E}^{\mathrm{f}}\left(\left(x_{1}-1, x_{2}\right), z\right) \frac{1+\varepsilon^{\mathrm{f}}\left(\left(x_{1}-1, x_{2}\right), z\right)}{\sqrt{\left(1+\mu^{\mathrm{f}}\left(x_{1}-1, x_{2}\right)\left(1+\mu^{\mathrm{f}}(z)\right)\right.}} f(z)\right. \\
& \left.-\frac{1}{m^{\mathrm{f}}(x)} \sum_{z \sim x} \mathcal{E}^{\mathrm{f}}(x, z) \frac{1+\varepsilon^{\mathrm{f}}(x, z)}{\sqrt{\left(1+\mu^{\mathrm{f}}(x)\right)\left(1+\mu^{\mathrm{f}}(z)\right)}} f\left(z_{1}-1, z_{2}\right)\right)\left.\right|^{2} \\
& +\sum_{x \in \mathcal{V}^{\mathrm{f}}} m^{\mathrm{f}}(x) \mid \frac{\mathrm{i}}{2}\left(e^{-1 / 2}\langle Q+1 / 2\rangle^{1+\epsilon} \otimes \mathbf{1}_{\mathcal{V}_{2}^{\mathrm{f}}}\right) \\
& \times\left(\frac{1}{m^{\mathrm{f}}\left(x_{1}+1, x_{2}\right)} \sum_{z \sim x} \mathcal{E}^{\mathrm{f}}\left(\left(x_{1}+1, x_{2}\right), z\right) \frac{1+\varepsilon^{\mathrm{f}}\left(\left(x_{1}+1, x_{2}\right), z\right)}{\sqrt{\left(1+\mu^{\mathrm{f}}\left(x_{1}+1, x_{2}\right)\left(1+\mu^{\mathrm{f}}(z)\right)\right.}}\right. \\
& \left.-\frac{1}{m^{\mathrm{f}}(x)} \sum_{z \sim x} \mathcal{E}^{\mathrm{f}}(x, z) \frac{1+\varepsilon^{\mathrm{f}}(x, z)}{\sqrt{\left(1+\mu^{\mathrm{f}}(x)\right)\left(1+\mu^{\mathrm{f}}(z)\right)}}\right)\left.f\left(x_{1}+1, x_{2}\right)\right|^{2} \\
& +\sum_{x \in \mathcal{V}^{\mathrm{f}}} m^{\mathrm{f}}(x) \mid \frac{\mathrm{i}}{2}\left(e^{-1 / 2}\langle Q+1 / 2\rangle^{1+\epsilon} \otimes \mathbf{1}_{\left.\mathcal{V}_{2}^{\mathrm{f}}\right)}\right. \\
& \times\left(\frac{1}{m^{\mathrm{f}}(x)} \sum_{z \sim x} \mathcal{E}^{\mathrm{f}}(x, z) \frac{1+\varepsilon^{\mathrm{f}}(x, z)}{\sqrt{\left(1+\mu^{\mathrm{f}}(x)\right)\left(1+\mu^{\mathrm{f}}(z)\right)}} f\left(z_{1}+1, z_{2}\right)\right. \\
& \left.+\frac{1}{m^{\mathrm{f}}\left(x_{1}+1, x_{2}\right)} \sum_{z \sim x} \mathcal{E}^{\mathrm{f}}\left(\left(x_{1}+1, x_{2}\right), z\right) \frac{1+\varepsilon^{\mathrm{f}}\left(\left(x_{1}+1, x_{2}\right), z\right)}{\sqrt{\left(1+\mu^{\mathrm{f}}\left(x_{1}+1, x_{2}\right)\left(1+\mu^{\mathrm{f}}(z)\right)\right.}} f\left(z_{1}, z_{2}\right)\right)\left.\right|^{2} \\
& +\left\|\left\langle\Lambda^{\mathrm{f}}\right\rangle^{\epsilon}\left[W^{\mathrm{f}}(\cdot), \mathcal{A}_{\mathcal{G}_{\mathrm{f}}}\right] f\right\|_{\ell^{2}\left(\mathcal{V}^{\mathrm{f}}, m^{\mathrm{f}}\right)},
\end{aligned}
$$

with

$$
\left[W^{\mathrm{f}}(\cdot), \mathcal{A}_{\mathcal{G}^{\mathrm{f}}}\right] f(x)=\frac{\mathrm{i}}{2} e^{1 / 2}\left((Q-1 / 2) \otimes \mathbf{1}_{\mathcal{V}_{2}^{\mathrm{f}}}\right) \frac{1}{m^{\mathrm{f}}(x)} \sum_{z \sim x}\left(1+\varepsilon^{\mathrm{f}}(x, z)\right) \mathcal{E}^{\mathrm{f}}(x, z) U f(x)
$$

$$
\times\left(\frac{\mu^{\mathrm{f}}(z)-\mu^{\mathrm{f}}(x)}{\left(1+\mu^{\mathrm{f}}(x)\right) \sqrt{1+\mu^{\mathrm{f}}(z)}\left(\sqrt{1+\mu^{\mathrm{f}}(z)}+\sqrt{1+\mu^{\mathrm{f}}(x)}\right)}\right)
$$




$$
\begin{aligned}
& \quad-\frac{\mathrm{i}}{2} e^{1 / 2}\left((Q-1 / 2) \otimes \mathbf{1}_{\mathcal{V}_{2}^{\mathrm{f}}}\right) \frac{1}{m^{\mathrm{f}}\left(x_{1}-1, x_{2}\right)} \sum_{z \sim x}\left(1+\varepsilon^{\mathrm{f}}\left(\left(x_{1}-1, x_{2}\right),\left(z_{1}-1, z_{2}\right)\right)\right. \\
& \times\left(\frac{\mu^{\mathrm{f}}\left(z_{1}-1, z_{2}\right)-\mu^{\mathrm{f}}\left(x_{1}-1, x_{2}\right)}{\left(1+\mu^{\mathrm{f}}\left(x_{1}-1, x_{2}\right)\right) \sqrt{1+\mu^{\mathrm{f}}\left(z_{1}-1, z_{2}\right)}\left(\sqrt{1+\mu^{\mathrm{f}}\left(z_{1}-1, z_{2}\right)}+\sqrt{1+\mu^{\mathrm{f}}\left(x_{1}-1, x_{2}\right)}\right)}\right) \\
& \quad \times \frac{\mathrm{i}}{2} e^{-1 / 2}\left((Q+1 / 2) \otimes \mathbf{1}_{\mathcal{V}_{2}^{\mathrm{f}}}\right) \frac{1}{m^{\mathrm{f}}\left(x_{1}+1, x_{2}\right)} \sum_{z \sim x}\left(1+\varepsilon^{\mathrm{f}}\left(\left(x_{1}+1, x_{2}\right),\left(z_{1}+1, z_{2}\right)\right)\right. \\
& \times\left(\frac{\mu^{\mathrm{f}}\left(z_{1}+1, z_{2}\right)-\mu^{\mathrm{f}}\left(x_{1}+1, x_{2}\right)}{\left(1+\mu^{\mathrm{f}}\left(x_{1}+1, x_{2}\right)\right) \sqrt{1+\mu^{\mathrm{f}}\left(z_{1}+1, z_{2}\right)}\left(\sqrt{1+\mu^{\mathrm{f}}\left(z_{1}+1, z_{2}\right)}+\sqrt{1+\mu^{\mathrm{f}}\left(x_{1}+1, x_{2}\right)}\right)}\right) \\
& \quad \times \mathcal{E}^{\mathrm{f}}\left(\left(x_{1}+1, x_{2}\right),\left(z_{1}+1, z_{2}\right)\right) U^{*} f(x) \\
& -\frac{\mathrm{i}}{2} e^{-1 / 2}\left((Q+1 / 2) \otimes \mathbf{1}_{\mathcal{V}_{2}^{\mathrm{f}}}\right) \frac{1}{m^{\mathrm{f}}(x)} \sum_{z \sim x}\left(1+\varepsilon^{\mathrm{f}}(x, z)\right) \mathcal{E}^{\mathrm{f}}(x, z) U^{*} f(x) \\
& \times\left(\frac{\mu^{\mathrm{f}}(z)-\mu^{\mathrm{f}}(x)}{\left(1+\mu^{\mathrm{f}}(x)\right) \sqrt{1+\mu^{\mathrm{f}}(z)}\left(\sqrt{1+\mu^{\mathrm{f}}(z)}+\sqrt{1+\mu^{\mathrm{f}}(x)}\right)}\right) .
\end{aligned}
$$

We treat the first term of $\left\|\left\langle\Lambda^{\mathrm{f}}\right\rangle^{\epsilon}\left[\widetilde{\Delta}_{\mathcal{G}_{\mathcal{E}, \mu}^{\mathrm{f}}}, \mathcal{A}_{\mathcal{G}^{\mathrm{f}}}\right] f\right\|_{\ell^{2}\left(\mathcal{V}^{\mathrm{f}}, m^{\mathrm{f}}\right)}$ in (4.5).

$$
\begin{gathered}
\sum_{x \in \mathcal{V}^{\mathrm{f}}} m^{\mathrm{f}}(x) \mid \frac{\mathrm{i}}{2}\left(e^{1 / 2}\langle Q-1 / 2\rangle^{1+\epsilon} \otimes \mathbf{1}_{\mathcal{V}_{2}^{\mathrm{f}}}\right) \sum_{z \sim x}\left(\frac{\mathcal{E}^{\mathrm{f}}(x, z)\left(1+\varepsilon^{\mathrm{f}}(x, z)\right)}{m^{\mathrm{f}}(x) \sqrt{\left(1+\mu^{\mathrm{f}}(x)\right)\left(1+\mu^{\mathrm{f}}(z)\right)}}\right. \\
\left.-\frac{\mathcal{E}^{\mathrm{f}}\left(\left(x_{1}-1, x_{2}\right), z\right)\left(1+\varepsilon^{\mathrm{f}}\left(\left(x_{1}-1, x_{2}\right), z\right)\right)}{m\left(x_{1}-1, x_{2}\right) \sqrt{\left(1+\mu^{\mathrm{f}}\left(x_{1}-1, x_{2}\right)\left(1+\mu^{\mathrm{f}}(z)\right)\right.}}\right)\left.f\left(x_{1}-1, x_{2}\right)\right|^{2}
\end{gathered}
$$

$$
\begin{aligned}
\leq & 2 \sum_{x \in \mathcal{V}^{\mathrm{f}}} m^{\mathrm{f}}(x) \mid \frac{\mathrm{i}}{2}\left(e^{1 / 2}\langle Q-1 / 2\rangle^{1+\epsilon} \otimes \mathbf{1}_{\mathcal{V}_{2}^{\mathrm{f}}}\right) \\
& \times \sum_{z_{1} \sim x_{1}} \delta_{z_{2}=x_{2}}\left(\frac{\mathcal{E}_{1}^{\mathrm{f}}\left(x_{1}, z_{1}\right)\left(1+\varepsilon^{\mathrm{f}}(x, z)\right)}{m(x) \sqrt{\left(1+\mu^{\mathrm{f}}(x)\right)\left(1+\mu^{\mathrm{f}}(z)\right)}}\right. \\
& \left.-\frac{\mathcal{E}_{1}^{\mathrm{f}}\left(x_{1}-1, z_{1}\right)\left(1+\varepsilon^{\mathrm{f}}\left(\left(x_{1}-1, x_{2}\right), z\right)\right)}{m^{\mathrm{f}}\left(x_{1}-1, x_{2}\right) \sqrt{\left(1+\mu^{\mathrm{f}}\left(x_{1}-1, x_{2}\right)\left(1+\mu^{\mathrm{f}}(z)\right)\right.}}\right)\left.f\left(x_{1}-1, x_{2}\right)\right|^{2} \\
+ & 2 \sum_{x \in \mathcal{V}^{\mathrm{f}}} m^{\mathrm{f}}(x) \mid \frac{\mathrm{i}}{2}\left(e^{1 / 2}\langle Q-1 / 2\rangle^{1+\epsilon} \otimes \mathbf{1}_{\mathcal{V}_{2}^{\mathrm{f}}}\right) \\
& \times \sum_{z_{2} \sim x_{2}} \delta_{z_{1}=x_{1}}\left(\frac{\mathcal{E}_{2}^{\mathrm{f}}\left(x_{2}, z_{2}\right)\left(1+\varepsilon^{\mathrm{f}}(x, z)\right)}{m(x) \sqrt{\left(1+\mu^{\mathrm{f}}(x)\right)\left(1+\mu^{\mathrm{f}}(z)\right)}}\right. \\
& \left.-\frac{\mathcal{E}_{2}^{\mathrm{f}}\left(x_{2}, z_{2}\right)\left(1+\varepsilon^{\mathrm{f}}\left(\left(x_{1}-1, x_{2}\right), z\right)\right)}{m_{1}^{\mathrm{f}}\left(x_{1}-1\right) m_{2} \sqrt{\left(1+\mu^{\mathrm{f}}\left(x_{1}-1, x_{2}\right)\left(1+\mu^{\mathrm{f}}(z)\right)\right.}}\right)\left.f\left(x_{1}-1, x_{2}\right)\right|^{2} .
\end{aligned}
$$


We bound (4.7) as follows:

$$
\leq 4 \sum_{x \in \mathcal{V}^{\mathrm{f}}} m^{\mathrm{f}}(x) \mid \frac{\mathrm{i}}{2}\left(e\langle Q-1 / 2\rangle^{1+\epsilon} \otimes \mathbf{1}_{\mathcal{V}_{2}^{\mathrm{f}}}\right) \frac{1}{m_{2}}
$$

$$
\begin{aligned}
& \times\left(\frac{\sqrt{1+\mu^{\mathrm{f}}\left(x_{1}-1, x_{2}\right)}\left(1+\varepsilon^{\mathrm{f}}\left(x,\left(x_{1}+1, x_{2}\right)\right)\right.}{\sqrt{\left(1+\mu^{\mathrm{f}}(x)\right)\left(1+\mu^{\mathrm{f}}\left(x_{1}+1, x_{2}\right)\right)\left(1+\mu^{\mathrm{f}}\left(x_{1}-1, x_{2}\right)\right)}}\right. \\
& \left.\quad-\frac{\sqrt{1+\mu^{\mathrm{f}}\left(x_{1}+1, x_{2}\right)}\left(1+\varepsilon^{\mathrm{f}}\left(\left(x_{1}-1, x_{2}\right),\left(x_{1}, x_{2}\right)\right)\right)}{\sqrt{\left(1+\mu^{\mathrm{f}}\left(x_{1}-1, x_{2}\right)\left(1+\mu^{\mathrm{f}}(x)\right)\left(1+\mu^{\mathrm{f}}\left(x_{1}+1, x_{2}\right)\right)\right.}}\right)\left.f\left(x_{1}-1, x_{2}\right)\right|^{2} \\
& \quad+4 \sum_{x \in \mathcal{V}^{\mathrm{f}}} m^{\mathrm{f}}(x) \mid \frac{\mathrm{i}}{2}\left(\langle Q-1 / 2\rangle^{1+\epsilon} \otimes \mathbf{1}_{\mathcal{V}_{2}^{\mathrm{f}}}\right) \frac{1}{m_{2}}
\end{aligned}
$$

$$
\begin{aligned}
& \times\left(\frac{\sqrt{1+\mu^{\mathrm{f}}\left(x_{1}-2, x_{2}\right)}\left(1+\varepsilon^{\mathrm{f}}\left(x,\left(x_{1}-1, x_{2}\right)\right)\right)}{\sqrt{\left(1+\mu^{\mathrm{f}}(x)\right)\left(1+\mu^{\mathrm{f}}\left(x_{1}-1, x_{2}\right)\right)\left(1+\mu^{\mathrm{f}}\left(x_{1}-2, x_{2}\right)\right)}}\right. \\
& \left.-\frac{\sqrt{1+\mu^{\mathrm{f}}\left(x_{1}-1, x_{2}\right)}\left(1+\varepsilon^{\mathrm{f}}\left(\left(x_{1}-1, x_{2}\right),\left(x_{1}-2, x_{2}\right)\right)\right)}{\sqrt{\left(1+\mu^{\mathrm{f}}\left(x_{1}-1, x_{2}\right)\left(1+\mu^{\mathrm{f}}\left(x_{1}-2, x_{2}\right)\right)\left(1+\mu^{\mathrm{f}}\left(x_{1}-1, x_{2}\right)\right)\right.}}\right)\left.f\left(x_{1}-1, x_{2}\right)\right|^{2},
\end{aligned}
$$

Now, we concentrate on (4.9) and in the same way, we deal with (4.10). Since the assertions $(H 2)$ and $(H 3)$ hold true then there exists an integer $c$, such that

$$
\begin{aligned}
4 & \sum_{x \in \mathcal{V}^{\mathrm{f}}} m^{\mathrm{f}}(x) \mid \frac{\mathrm{i}}{2}\left(e\langle Q-1 / 2\rangle^{1+\epsilon} \otimes \mathbf{1}_{\mathcal{V}_{2}^{\mathrm{f}}}\right) \\
& \times \frac{1}{m_{2}}\left(\frac{\sqrt{1+\mu^{\mathrm{f}}\left(x_{1}-1, x_{2}\right)}\left(1+\varepsilon^{\mathrm{f}}\left(x,\left(x_{1}+1, x_{2}\right)\right)\right.}{\sqrt{\left(1+\mu^{\mathrm{f}}(x)\right)\left(1+\mu^{\mathrm{f}}\left(x_{1}+1, x_{2}\right)\right)\left(1+\mu^{\mathrm{f}}\left(x_{1}-1, x_{2}\right)\right)}}\right. \\
& \left.-\frac{\sqrt{1+\mu^{\mathrm{f}}\left(x_{1}+1, x_{2}\right)}\left(1+\varepsilon^{\mathrm{f}}\left(\left(x_{1}-1, x_{2}\right),\left(x_{1}, x_{2}\right)\right)\right)}{\sqrt{\left(1+\mu^{\mathrm{f}}\left(x_{1}-1, x_{2}\right)\left(1+\mu^{\mathrm{f}}(x)\right)\left(1+\mu^{\mathrm{f}}\left(x_{1}+1, x_{2}\right)\right)\right.}}\right)\left.f\left(x_{1}-1, x_{2}\right)\right|^{2} \\
= & 4 \sum_{x \in \mathcal{V}^{\mathrm{f}}} m^{\mathrm{f}}(x) \mid \frac{\mathrm{i}}{2}\left(e\langle Q-1 / 2\rangle^{1+\epsilon} \otimes \mathbf{1}_{\mathcal{V}_{2}^{\mathrm{f}}}\right) \frac{1}{m_{2}} \\
& \times\left(\frac{\left(\mu^{\mathrm{f}}\left(x_{1}-1, x_{2}\right)-\mu^{\mathrm{f}}\left(x_{1}+1, x_{2}\right)\right) \varepsilon^{\mathrm{f}}\left(x,\left(x_{1}+1, x_{2}\right)\right)}{\left(\sqrt{1+\mu^{\mathrm{f}}\left(x_{1}-1, x_{2}\right)}+\sqrt{1+\mu^{\mathrm{f}}\left(x_{1}+1, x_{2}\right)}\right)}\right. \\
& \times \frac{1}{\sqrt{\left(1+\mu^{\mathrm{f}}(x)\right)\left(1+\mu^{\mathrm{f}}\left(x_{1}+1, x_{2}\right)\right)\left(1+\mu^{\mathrm{f}}\left(x_{1}-1, x_{2}\right)\right)}} \\
& +\frac{\sqrt{1+\mu^{\mathrm{f}}\left(x_{1}+1, x_{2}\right)}\left(\varepsilon^{\mathrm{f}}\left(x,\left(x_{1}+1, x_{2}\right)\right)-\varepsilon^{\mathrm{f}}\left(\left(x_{1}-1, x_{2}\right), x\right)\right.}{\sqrt{\left(1+\mu^{\mathrm{f}}(x)\right)\left(1+\mu^{\mathrm{f}}\left(x_{1}+1, x_{2}\right)\right)\left(1+\mu^{\mathrm{f}}\left(x_{1}-1, x_{2}\right)\right)}} \\
& \left.+\frac{\mu^{\mathrm{f}}\left(x_{1}-1, x_{2}\right)-\mu^{\mathrm{f}}\left(x_{1}+1, x_{2}\right)}{\sqrt{\left(1+\mu^{\mathrm{f}}(x)\right)\left(1+\mu^{\mathrm{f}}\left(x_{1}+1, x_{2}\right)\right)\left(1+\mu^{\mathrm{f}}\left(x_{1}-1, x_{2}\right)\right)}}\right)\left.f\left(x_{1}-1, x_{2}\right)\right|^{2} \\
& \times \sqrt{\ell^{2}\left(\mathcal{V}^{\mathrm{f}}, m^{\mathrm{f}}\right)} \cdot
\end{aligned}
$$

In the same way, we treat (4.8) and $\left\|\left\langle\Lambda^{\mathrm{f}}\right\rangle^{\epsilon}\left[W^{\mathrm{f}}(\cdot), \mathcal{A}_{\mathcal{G}^{\mathrm{f}}}\right] f\right\|_{\ell^{2}\left(\mathcal{V}^{\mathrm{f}}, m^{\mathrm{f}}\right)}^{2}$. By density, there exists $c>0$ such that $\left\|\left\langle\Lambda^{\mathrm{f}}\right\rangle^{\epsilon}\left[\widetilde{\Delta}_{\mathcal{G}_{\varepsilon, \mu}^{\mathrm{f}}}, \mathcal{A}_{\mathcal{G}^{\mathrm{f}}}\right] f\right\|_{\ell^{2}\left(\mathcal{V}^{\mathrm{f}}, m^{\mathrm{f}}\right)}^{2} \leq c\|f\|_{\ell^{2}\left(\mathcal{V}^{\mathrm{f}}, m^{\mathrm{f}}\right)}^{2}$. Finally, by applying [AmBoGe, Proposition 7.5.7] where the hypotheses are verified in Proposition 4.6 , we find the result. 
4.4. The cusp side: Radial metric perturbation. We recall that

$$
\widetilde{\Delta}_{\mathcal{G}_{\varepsilon, \mu}^{\mathrm{c}}} f(x):=T_{m_{\mu} \rightarrow m} \Delta_{\mathcal{G}_{\varepsilon, \mu}^{\mathrm{c}}} T_{m_{\mu} \rightarrow m}^{-1} f(x), \quad \text { for all } \mathrm{f} \in \mathcal{C}_{\mathrm{c}}\left(\mathcal{V}^{\mathrm{c}}\right) .
$$

We first deal with the question of the essential spectrum.

Proposition 4.9. Let $V^{\mathrm{c}}: \mathcal{V}^{\mathrm{c}} \rightarrow \mathbb{R}$ be a function obeying $V^{\mathrm{c}}(x) \rightarrow 0$ if $|x| \rightarrow \infty$. We assume that (4.1) holds true then $\widetilde{\Delta}_{\mathcal{G}_{\varepsilon, \mu}^{\mathrm{c}}}-\Delta_{\mathcal{G}^{\mathrm{c}}} \in \mathcal{K}\left(\ell^{2}\left(\mathcal{V}^{\mathrm{c}}, m^{\mathrm{c}}\right)\right)$. In particular,

(1) $\mathcal{D}\left(\Delta_{\mathcal{G}_{\varepsilon, \mu}^{\mathrm{c}}}+V(\cdot)\right)=\mathcal{D}\left(T_{m_{\mu} \rightarrow m}^{-1} \Delta_{\mathcal{G}} T_{m_{\mu} \rightarrow m}\right)$,

(2) $\Delta_{\mathcal{G}_{\varepsilon, \mu}^{\mathrm{c}}}+V(\cdot)$ is essentially self-adjoint on $\mathcal{C}_{c}(\mathcal{V})$,

(3) $\sigma_{\mathrm{ess}}\left(\Delta_{\mathcal{G}_{\varepsilon, \mu}^{\mathrm{c}}}+V(\cdot)\right)=\sigma_{\mathrm{ess}}\left(\Delta_{\mathcal{G}}\right)$.

Proof. Let $f \in \mathcal{C}_{c}\left(\mathcal{V}^{\mathrm{c}}\right)$, we have

$$
\begin{aligned}
& \left|\left\langle f,\left(\widetilde{\Delta}_{\mathcal{G}_{\varepsilon, \mu}^{\mathrm{c}}}-\Delta_{\mathcal{G}^{\mathrm{c}}}\right) f\right\rangle_{\ell^{2}\left(\mathcal{V}^{\mathrm{c}}, m^{\mathrm{c}}\right)}\right|=\left|\sum_{x \in \mathcal{V}^{\mathrm{c}}} m^{\mathrm{c}}(x)\left(\widetilde{\Delta}_{\mathcal{G}_{\varepsilon, \mu}^{\mathrm{c}}}-\Delta_{\mathcal{G}^{\mathrm{c}}}\right) f(x) \overline{f(x)}\right| \\
& \leq \sum_{x \in \mathcal{V}^{\mathrm{c}}} m^{\mathrm{c}}(x) \frac{1}{m^{\mathrm{c}}(x)} \sum_{z_{1} \sim x_{1}} \frac{\varepsilon^{\mathrm{c}}\left(x,\left(z_{1}, x_{2}\right)\right)}{\sqrt{\left(1+\mu^{\mathrm{c}}(x)\right)\left(1+\mu^{\mathrm{c}}\left(z_{1}, x_{2}\right)\right)}} \\
& \times \mathcal{E}_{1}^{\mathrm{c}}\left(x_{1}, z_{1}\right)|f(x)|^{2} \\
& +1 / 2 \sum_{x \in \mathcal{V}^{\mathrm{c}}} m^{\mathrm{c}}(x) \frac{1}{m^{\mathrm{c}}(x)} \sum_{z_{1} \sim x_{1}} \frac{\varepsilon^{\mathrm{c}}\left(x,\left(z_{1}, x_{2}\right)\right)}{\sqrt{\left(1+\mu^{\mathrm{c}}(x)\right)\left(1+\mu^{\mathrm{c}}\left(z_{1}, x_{2}\right)\right)}} \\
& \times \mathcal{E}^{\mathrm{c}}(x, z)\left(|f(z)|^{2}+|f(x)|^{2}\right) \\
& +\sum_{x \in \mathcal{V}^{\mathrm{c}}} m^{\mathrm{c}}(x) \frac{1}{m^{\mathrm{c}}(x)} \sum_{z_{1} \sim x_{1}}\left|\frac{\mu^{\mathrm{c}}(x)+\mu^{\mathrm{c}}\left(z_{1}, x_{2}\right)+\mu^{\mathrm{c}}(x) \mu^{\mathrm{c}}\left(z_{1}, x_{2}\right)}{\sqrt{\left(1+\mu^{\mathrm{c}}(x)\right)\left(1+\mu^{\mathrm{c}}\left(z_{1}, x_{2}\right)\right)}}\right| \\
& \times \frac{1}{\sqrt{1+\mu^{\mathrm{c}}(x)}+\sqrt{1+\mu^{\mathrm{c}}\left(z_{1}, x_{2}\right)}} \mathcal{E}_{1}^{\mathrm{c}}\left(x_{1}, z_{1}\right)|f(x)|^{2} \\
& +1 / 2 \sum_{x \in \mathcal{V}^{\mathrm{c}}} m^{\mathrm{c}}(x) \frac{1}{m^{\mathrm{c}}(x)} \sum_{z_{1} \sim x_{1}}\left|\frac{\mu^{\mathrm{c}}(x)+\mu^{\mathrm{c}}\left(z_{1}, x_{2}\right)+\mu^{\mathrm{c}}(x) \mu^{\mathrm{c}}\left(z_{1}, x_{2}\right)}{\sqrt{\left(1+\mu^{\mathrm{c}}(x)\right)\left(1+\mu^{\mathrm{c}}\left(z_{1}, x_{2}\right)\right)}}\right| \\
& \times \frac{1}{\sqrt{1+\mu^{\mathrm{c}}(x)}+\sqrt{1+\mu^{\mathrm{c}}\left(z_{1}, x_{2}\right)}} \mathcal{E}_{1}^{\mathrm{c}}\left(x_{1}, z_{1}\right)\left|f\left(z_{1}, x_{2}\right)\right|^{2} \\
& +1 / 2 \sum_{x \in \mathcal{V}^{\mathrm{c}}} m^{\mathrm{c}}(x) \frac{1}{m^{\mathrm{c}}(x)} \sum_{z_{1} \sim x_{1}}\left|\frac{\mu^{\mathrm{c}}(x)+\mu^{\mathrm{c}}\left(z_{1}, x_{2}\right)+\mu^{\mathrm{c}}(x) \mu^{\mathrm{c}}\left(z_{1}, x_{2}\right)}{\sqrt{\left(1+\mu^{\mathrm{c}}(x)\right)\left(1+\mu^{\mathrm{c}}\left(z_{1}, x_{2}\right)\right)}}\right| \\
& \times \frac{1}{\sqrt{1+\mu^{\mathrm{c}}(x)}+\sqrt{1+\mu^{\mathrm{c}}\left(z_{1}, x_{2}\right)}} \mathcal{E}_{1}^{\mathrm{c}}\left(x_{1}, z_{1}\right)|f(x)|^{2} \\
& \leq 2\left\langle f,\left(\operatorname{deg}_{3}(\cdot)+\operatorname{deg}_{4}(\cdot)+\left|W^{\mathrm{c}}(\cdot)\right|\right) f\right\rangle \text {, }
\end{aligned}
$$

where

$$
\operatorname{deg}_{3}(x):=\frac{1}{m^{\mathrm{c}}(x)} \sum_{z_{1} \in \mathcal{V}_{1}^{\mathrm{c}}} \frac{\varepsilon^{\mathrm{c}}\left(x,\left(z_{1}, x_{2}\right)\right)}{\sqrt{\left(1+\mu^{\mathrm{c}}(x)\right)\left(1+\mu^{\mathrm{c}}\left(z_{1}, x_{2}\right)\right)}} \mathcal{E}_{1}^{\mathrm{c}}\left(x_{1}, z_{1}\right)
$$

and

$$
\begin{gathered}
\operatorname{deg}_{4}(x):=\frac{1}{m^{\mathrm{c}}(x)} \sum_{z_{1} \in \mathcal{V}_{1}^{\mathrm{c}}} \mid \frac{\mu^{\mathrm{c}}(x)+\mu^{\mathrm{c}}\left(z_{1}, x_{2}\right)+\mu^{\mathrm{c}}(x) \mu^{\mathrm{c}}\left(z_{1}, x_{2}\right)}{\sqrt{\left(1+\mu^{\mathrm{c}}(x)\right)\left(1+\mu^{\mathrm{c}}\left(z_{1}, x_{2}\right)\right)}\left(\sqrt{1+\mu^{\mathrm{c}}(x)}+\sqrt{1+\mu^{\mathrm{c}}\left(z_{1}, x_{2}\right)}\right)} \\
\times \mathcal{E}_{1}^{\mathrm{c}}\left(x_{1}, z_{1}\right) .
\end{gathered}
$$

We have

$$
\left|\operatorname{deg}_{3}(x)\right| \leq \sup _{z_{1} \sim x_{1}}\left|\frac{\varepsilon^{\mathrm{c}}\left(x,\left(z_{1}, x_{2}\right)\right)}{m_{2} \sqrt{\left(1+\mu^{\mathrm{c}}(x)\right)\left(1+\mu^{\mathrm{c}}\left(z_{1}, x_{2}\right)\right)}}\right| \operatorname{deg}_{\mathcal{G}_{1}^{\mathrm{c}}}(x) .
$$


Since $V_{2}$ is a finite set and for all $x_{2} \in \mathcal{V}_{2}, \frac{\varepsilon^{\mathrm{c}}\left(\left(x_{1}, x_{2}\right),\left(z_{1}, x_{2}\right)\right)}{\sqrt{\left(1+\mu^{\mathrm{c}}\left(x_{1}, x_{2}\right)\right)\left(1+\mu^{\mathrm{c}}\left(z_{1}, x_{2}\right)\right)}} \rightarrow 0$ when $x_{1}, z_{1} \rightarrow \infty$ and since $\operatorname{deg}_{\mathcal{G}_{1}^{\mathrm{c}}}(\cdot)$ is bounded then $\operatorname{deg}_{3}(\cdot)$ is compact. In the same way, using that $\forall x_{2} \in \mathcal{V}_{2}, \frac{\mu^{\mathrm{c}}(x)+\mu^{\mathrm{c}}\left(z_{1}, x_{2}\right)+\mu^{\mathrm{c}}(x) \mu^{\mathrm{c}}\left(z_{1}, x_{2}\right)}{\sqrt{\left(1+\mu^{\mathrm{c}}\left(x_{1}, x_{2}\right)\right)\left(1+\mu^{\mathrm{c}}\left(z_{1}, x_{2}\right)\right)}\left(\sqrt{1+\mu^{\mathrm{c}}(x)}+\sqrt{1+\mu^{\mathrm{c}}\left(z_{1}, x_{2}\right)}\right)} \rightarrow 0$ if $x_{1}, z_{1} \rightarrow \infty$, we obtain the compactness of $\operatorname{deg}_{4}(\cdot)$.

Now, we will show that $W^{\mathrm{c}}(\cdot) \in \mathcal{K}\left(\ell^{2}\left(\mathcal{V}^{\mathrm{c}}, m^{\mathrm{c}}\right)\right.$. For all $x \in \mathcal{V}^{\mathrm{c}}$, we have

$$
\begin{aligned}
& \left|W^{\mathrm{c}}(x)\right|=\mid \frac{1}{m^{\mathrm{c}}(x)} \sum_{z \sim x}\left(\frac{\mu^{\mathrm{c}}(z)-\mu^{\mathrm{c}}(x)}{\left(1+\mu^{\mathrm{c}}(x)\right) \sqrt{1+\mu^{\mathrm{c}}(z)}\left(\sqrt{1+\mu^{\mathrm{c}}(z)}+\sqrt{1+\mu^{\mathrm{c}}(x)}\right)}\right) \\
& \quad \times\left(1+\varepsilon^{\mathrm{c}}(x, z)\right) \mathcal{E}^{\mathrm{c}}(x, z) \mid \\
& \leq \sup _{z_{1} \sim x_{1}}\left(1+\varepsilon^{\mathrm{c}}\left(x,\left(z_{1}, x_{2}\right)\right)\right) \operatorname{deg}_{\mathcal{G}_{1}^{\mathrm{c}}}(x) \\
& \quad \times\left|\frac{\mu^{\mathrm{c}}\left(z_{1}, x_{2}\right)-\mu^{\mathrm{c}}(x)}{\left(1+\mu^{\mathrm{c}}(x)\right) \sqrt{1+\mu^{\mathrm{c}}\left(z_{1}, x_{2}\right)}\left(\sqrt{1+\mu^{\mathrm{c}}\left(z_{1}, x_{2}\right)}+\sqrt{1+\mu^{\mathrm{c}}(x)}\right)}\right|
\end{aligned}
$$

Since $\mathcal{V}_{2}$ is a finite set and $\forall x_{2} \in \mathcal{V}_{2}, \varepsilon^{\mathrm{c}}\left(\left(x_{1}, x_{2}\right),\left(z_{1}, x_{2}\right)\right)\left(\mu^{\mathrm{c}}\left(z_{1}, x_{2}\right)-\mu^{\mathrm{c}}\left(x_{1}, x_{2}\right)\right) \rightarrow 0$ when $x_{1}, z_{1} \rightarrow \infty$, and since $\operatorname{deg}_{\mathcal{G}_{1}^{\mathrm{c}}}(\cdot)$ is bounded and since $V^{\mathrm{c}}(\cdot)$ is a compact perturbation. Then, $\widetilde{\Delta}_{\mathcal{G}_{\varepsilon, \mu}^{\mathrm{c}}}-\Delta_{\mathcal{G}^{\mathrm{c}}}$ is a compact operator. The points (1) and (2) follow from Theorem [ReSi, Theorem XIII.14] and (3) from the Weyl's Theorem.

In order to go into the Mourre theory, we construct the conjugate operator:

$$
\mathcal{A}_{\mathcal{G}^{\mathrm{c}}}:=\mathcal{A}_{m_{1}} \otimes P^{\mathrm{le}}
$$

with

$$
\begin{aligned}
\mathcal{A}_{m_{1}} & :=T_{1 \rightarrow m_{1}} \mathcal{A}_{\mathbb{N}} T_{1 \rightarrow m_{1}}^{-1} \\
& :=\frac{\mathrm{i}}{2}\left(e^{1 / 2}(Q-1 / 2) U-e^{-1 / 2}(Q+1 / 2) U^{*}\right) .
\end{aligned}
$$

It is self-adjoint and essentially self-adjoint on $\mathcal{C}^{c}\left(\mathcal{V}^{c}\right)$ by Lemma 3.2. Because of the projection in (4.11), we restrict to radial perturbations.

Definition 4.10. The perturbations $V^{\mathrm{c}}, \mu$ and $\varepsilon$ are called radial if they do not depend on the second variable, i.e., For all $\left(x_{1}, x_{2}\right),\left(z_{1}, z_{2}\right) \in \mathcal{V}^{\mathrm{c}}$, we have $V^{\mathrm{c}}\left(x_{1}, x_{2}\right)=$ $V^{\mathrm{c}}\left(x_{1}, z_{2}\right), \mu\left(x_{1}, x_{2}\right)=\mu\left(x_{1}, z_{2}\right)$ and $\varepsilon\left(\left(x_{1}, x_{2}\right),\left(z_{1}, z_{2}\right)\right)=\varepsilon\left(\left(x_{1}, x_{2}\right),\left(z_{1}, x_{2}\right)\right)$.

We turn to Mourre estimate.

Proposition 4.11. Let $\mathcal{G}_{\varepsilon, \mu}^{\mathrm{c}}$ a graph satisfies a condition (4.1). Suppose that $V^{\mathrm{c}}$ : $\mathcal{V}^{\mathrm{c}} \rightarrow \mathbb{R}$, $\varepsilon$ and $\mu$ are radial and assume (H1), (H2), and (H3) and $V^{\mathrm{c}}(x) \rightarrow 0$ if $|x| \rightarrow \infty$. Then $\Delta_{\mathcal{G}_{\varepsilon, \mu}^{\mathrm{c}}}+V^{\mathrm{c}}(\cdot) \in \mathcal{C}^{1,1}\left(\mathcal{A}_{\mathcal{G}_{\varepsilon, \mu}^{\mathrm{c}}}\right)$. Moreover, for all compact interval $I \subset\left(\frac{\alpha}{m_{2}}, \frac{\beta}{m_{2}}\right)$ there are $c>0$, a compact operator $K$ such that

$$
E_{I}\left(\Delta_{\mathcal{G}_{\varepsilon, \mu}^{\mathrm{c}}}+V^{\mathrm{c}}(\cdot)\right)\left[\Delta_{\mathcal{G}_{\varepsilon, \mu}^{\mathrm{c}}}+V^{\mathrm{c}}(\cdot), \mathrm{i} \mathcal{A}_{\mathcal{G}_{\varepsilon, \mu}^{\mathrm{c}}}\right]_{\circ} E_{I}\left(\Delta_{\mathcal{G}_{\varepsilon, \mu}^{\mathrm{c}}}+V^{\mathrm{c}}(\cdot)\right)
$$

in the form sense.

Proof. The Proposition 4.14 and Lemma 4.13 gives that $\Delta_{\mathcal{G}_{\varepsilon, \mu}^{\mathrm{c}}} \in \mathcal{C}^{1,1}\left(\mathcal{A}_{\mathcal{G}^{\mathrm{c}}}\right)$. Since $\widetilde{\Delta}_{\mathcal{G}_{\varepsilon, \mu}^{\mathrm{c}}}-\Delta_{\mathcal{G}^{\mathrm{c}}}$ is a compact operator by Proposition 4.9 , thanks to $(3.25)$ and by [AmBoGe, Theorem 7.2.9] we obtain (4.12).

We turn to series of Lemmata. To be able to apply the [AmBoGe, Proposition 7.5.7], we check the next point.

Proposition 4.12. Let $\Lambda^{\mathrm{c}}:=(Q+1 / 2) \otimes \mathbf{1}_{\mathcal{V}^{\mathrm{c}}}$, then $\Lambda^{\mathrm{c}}$ satisfies the following assertions:

(1) $e^{\mathrm{i} \Lambda^{\mathrm{c}} t} \mathcal{D}\left(\Delta_{\mathcal{G}_{\varepsilon, \mu}^{\mathrm{c}}}\right) \subset \mathcal{D}\left(\Delta_{\mathcal{G}_{\varepsilon, \mu}^{\mathrm{c}}}\right)$ and there exists a finite constant $\mathrm{c}$, such that

$$
\left\|e^{\mathrm{i} \Lambda^{\mathrm{c}} t}\right\|_{\mathcal{B}\left(\mathcal{D}\left(\Delta_{\mathcal{G}_{\varepsilon}^{\mathrm{c}}, \mu}\right)\right)} \leq \mathrm{c}, \quad \text { for all } \mathrm{t} \in \mathbb{R}
$$


(2) $\mathcal{D}\left(\Lambda^{\mathrm{c}}\right) \subset \mathcal{D}\left(\mathcal{A}_{\mathcal{G}_{\varepsilon, \mu}^{\mathrm{c}}}\right)$.

(3) $\left(\Lambda^{\mathrm{c}}\right)^{-2}\left(\mathcal{A}_{\mathcal{G}_{\varepsilon, \mu}^{\mathrm{c}}}\right)^{2}$ extends to a continuous operator in $\mathcal{D}\left(\Delta_{\mathcal{G}_{\varepsilon, \mu}^{\mathrm{c}}}\right)$.

Proof. With the help of the unitary transformation $T_{m_{\mu} \rightarrow m}$, it is enough to prove the result with $\varepsilon=0$ and $\mu=0$.

(1) We have

$$
\left[\Delta_{\mathcal{G}^{\mathrm{c}}}, e^{\mathrm{i} \Lambda^{\mathrm{c}} t}\right]=\left[\Delta_{\mathcal{G}_{1}^{\mathrm{c}}}, e^{\mathrm{i} \Lambda^{\mathrm{c}} t}\right] \otimes \frac{1}{m_{2}}+\left[\frac{1}{m_{1}(\cdot)}, e^{\mathrm{i} \Lambda^{\mathrm{c}} t}\right] \otimes \Delta_{2} .
$$

Since $\frac{1}{m_{1}(\cdot)}$ and $e^{\mathrm{i} \Lambda^{\mathrm{c}} t}$ commute and since $\left[\Delta_{\mathcal{G}_{1}^{\mathrm{c}}}, e^{\mathrm{i} \Lambda^{\mathrm{c}} t}\right]$ is uniformly bounded, then there exists $c>0$ such that for all $f \in \mathcal{C}_{c}\left(\mathcal{V}^{c}\right)$

$$
\left\|\left(\Delta_{\mathcal{G}^{c}}+\mathrm{i}\right) \mathrm{e}^{\mathrm{i} \Lambda^{\mathrm{c}} \mathrm{t}}\left(\Delta_{\mathcal{G}^{\mathrm{c}}}+\mathrm{i}\right)^{-1} \mathrm{f}\right\|_{\ell^{2}\left(\mathcal{V}^{\mathrm{c}}, \mathrm{m}^{\mathrm{c}}\right)} \leq \mathrm{c}\|\mathrm{f}\|_{\ell^{2}\left(\mathcal{V}^{\mathrm{c}}, \mathrm{m}^{\mathrm{c}}\right)} .
$$

Hence, there exists $c>0$ such that for all $f \in \mathcal{C}_{c}\left(\mathcal{V}^{c}\right)$

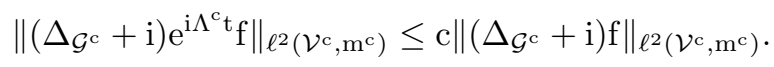

Since $\Delta_{\mathcal{G}^{c}}$ is essentially self-adjoint on $\mathcal{C}_{c}\left(\mathcal{V}^{c}\right)$ then we find the result.

(2) Let $f \in \mathcal{C}_{c}\left(\mathcal{V}^{c}\right)$, by using the relations of Subsection 3.5, we have

$$
\begin{aligned}
\left\|\mathcal{A}_{\mathcal{G}^{\mathrm{c}}} f\right\|_{\ell^{2}\left(\mathcal{V}^{\mathrm{c}}, m^{\mathrm{c}}\right)}^{2} \leq & \frac{1}{2} \sum_{x \in \mathcal{V}^{\mathrm{f}}} m^{\mathrm{f}}(x)\left|e^{1 / 2}(Q-1 / 2) U \otimes P^{\mathrm{le}} f(x)\right|^{2} \\
& +\left|e^{-1 / 2}(Q+1 / 2) U^{*} \otimes P^{\mathrm{le}} f(x)\right|^{2} \\
\leq & c \sum_{x \in \mathcal{V}^{\mathrm{f}}} m^{\mathrm{f}}(x)\left|(Q+1 / 2) \otimes P^{\mathrm{le}} f(x)\right|^{2} \leq c\left\|\Lambda^{\mathrm{c}} f\right\|_{\ell^{2}\left(\mathcal{V}^{\mathrm{f}}, m^{\mathrm{f}}\right)}^{2} .
\end{aligned}
$$

Since, $\Lambda^{\mathrm{c}}$ is essentially self-adjoint on $\mathcal{C}_{c}\left(\mathcal{V}^{\mathrm{c}}\right)$. we find the result.

(3) First for all $f \in \mathcal{C}_{c}\left(\mathcal{V}^{\mathrm{c}}\right)$, we have

$$
\begin{aligned}
\| & \left(\Lambda^{\mathrm{c}}\right)^{-2}\left(\mathcal{A}_{\mathcal{G}^{c}}\right)^{2} f \|_{\ell^{2}\left(\mathcal{V}^{\mathrm{c}}, m^{\mathrm{c}}\right)}^{2}=\sum_{\left(x_{1}, x_{2}\right) \in \mathcal{V}^{\mathrm{c}}} m^{\mathrm{c}}\left(x_{1}, x_{2}\right)\left|\Lambda^{-2}\left(\mathcal{A}_{\mathcal{G}^{c}}\right)^{2} f(x, y)\right|^{2} \\
= & \sum_{\left(x_{1}, x_{2}\right) \in \mathcal{V}^{\mathrm{c}}} m^{\mathrm{c}}\left(x_{1}, x_{2}\right) \mid \frac{1}{4}\left(\left((Q+1 / 2)^{-2}\left(2 Q^{2}+1 / 2\right)\right) \otimes P^{\mathrm{le}}\right) f\left(x_{1}, x_{2}\right) \\
& -\frac{1}{4} e\left(\left((Q+1 / 2)^{-2}(Q-1 / 2)(Q-3 / 2)\right) \otimes P^{\mathrm{le}}\right) f\left(x_{1}-2, x_{2}\right) \\
& -\left.\frac{1}{4} e^{-1}\left(\left((Q+1 / 2)^{-2}(Q+1 / 2)(Q+3 / 2)\right) \otimes P^{\mathrm{le}}\right) f\left(x_{1}+2, x_{2}\right)\right|^{2} .
\end{aligned}
$$

By density, we get $\left(\Lambda^{\mathrm{c}}\right)^{-2}\left(\mathcal{A}_{\mathcal{G}^{\mathrm{c}}}\right)^{2}$ is a bounded operator. Since $\Lambda^{\mathrm{c}}$ is a radial operator and $\Delta_{\mathcal{G}_{1}^{c}}$ is bounded then there exists $C>0$ such that, for all $f \in \mathcal{C}_{c}\left(\mathcal{V}^{c}\right)$,

$$
\begin{aligned}
\|\left[\Delta_{\mathcal{G}^{c}},\right. & \left.\left(\Lambda^{\mathrm{c}}\right)^{-2}\left(\mathcal{A}_{\mathcal{G}^{c}}\right)^{2}\right] f \|_{\ell^{2}\left(\mathcal{V}^{\mathrm{c}}, m^{\mathrm{c}}\right)} \\
& =\left\|\left(\left[\Delta_{\mathcal{G}_{1}^{\mathrm{c}}} \otimes \frac{1}{m_{2}},\left(\Lambda^{\mathrm{c}}\right)^{-2}\left(\mathcal{A}_{\mathcal{G}^{c}}\right)^{2}\right]+\left[\frac{1}{m_{1}(\cdot)} \otimes \Delta_{\mathcal{G}_{2}^{\mathrm{c}}},\left(\Lambda^{\mathrm{c}}\right)^{-2}\left(\mathcal{A}_{\mathcal{G}^{c}}\right)^{2}\right]\right) f\right\|_{\ell^{2}\left(\mathcal{V}^{c}, m^{\mathrm{c}}\right)} \\
& =\left\|\left(\left[\Delta_{\mathcal{G}_{1}^{\mathrm{c}}} \otimes \frac{1}{m_{2}},\left(\Lambda^{\mathrm{c}}\right)^{-2}\left(\mathcal{A}_{\mathcal{G}^{c}}\right)^{2}\right]+\left(\Lambda^{\mathrm{c}}\right)^{-2}\left[\frac{1}{m_{1}(\cdot)} \otimes \Delta_{\mathcal{G}_{2}^{\mathrm{c}}},\left(\mathcal{A}_{\mathcal{G}^{c}}\right)^{2}\right]\right) f\right\|_{\ell^{2}\left(\mathcal{V}^{\mathrm{c}}, m^{\mathrm{c}}\right)} \\
& =\left\|\left[\Delta_{\mathcal{G}_{1}^{\mathrm{c}}} \otimes \frac{1}{m_{2}},\left(\Lambda^{\mathrm{c}}\right)^{-2}\left(\mathcal{A}_{\mathcal{G}^{\mathrm{c}}}\right)^{2}\right] f\right\|_{\ell^{2}\left(\mathcal{V}^{\mathrm{c}}, m^{\mathrm{c}}\right)} \leq C\|f\|_{\ell^{2}\left(\mathcal{V}^{\mathrm{c}}, m^{\mathrm{c}}\right)} .
\end{aligned}
$$

We have used $\left[\frac{1}{m_{1}(\cdot)} \otimes \Delta_{\mathcal{G}_{2}^{c}},\left(\mathcal{A}_{\mathcal{G}^{c}}\right)^{2}\right]=0$ by construction. Conclude by density.

The proof of Proposition 4.14 is long and technical. For the sake of the reader, we have separated the treatment of the potential $V^{\mathrm{c}}$ to present the technical steps.

Lemma 4.13. Let $V^{\mathrm{c}}: \mathcal{V}^{\mathrm{c}} \rightarrow \mathbb{R}$ be a radial function and $(H 1)$ holds true, then $\left[V^{\mathrm{c}}(\cdot), \mathcal{A}_{\mathcal{G}_{\varepsilon, \mu}^{\mathrm{c}}}\right]_{\circ} \in \mathcal{C}^{0,1}\left(\mathcal{A}_{\mathcal{G}_{\varepsilon, \mu}^{\mathrm{c}}}\right)$. In particular, $V^{\mathrm{c}}(\cdot) \in \mathcal{C}^{1,1}\left(\mathcal{A}_{\mathcal{G}_{\varepsilon, \mu}^{\mathrm{c}}}\right)$. 
Proof. Since $V^{\mathrm{c}}$ is radial, by a slight abuse of notation, we have $V^{\mathrm{c}}:=V^{\mathrm{c}} \otimes \mathbf{1}_{\mathcal{V}_{2}}$. We compute the commutator on $\mathcal{C}^{c}\left(\mathcal{V}^{c}\right)$ and get

$$
\left[V^{\mathrm{c}}(\cdot), \mathrm{i} \mathcal{A}_{\mathcal{G}_{\varepsilon, \mu}^{\mathrm{c}}}\right]=\left(\frac{e^{-1 / 2}}{2}\left(Q+\frac{1}{2}\right)\left[V^{\mathrm{c}}, U^{*}\right]+\frac{e^{1 / 2}}{2}\left(\frac{1}{2}-Q\right)\left[V^{\mathrm{c}}, U\right]\right) \otimes P^{\mathrm{le}}
$$

By density, we infer that $\left[V^{\mathrm{c}}(\cdot), \mathrm{i} \mathcal{A}_{\mathcal{G}_{\varepsilon, \mu}^{\mathrm{c}}}\right]$ extends to a bounded operator and that $V^{\mathrm{c}}(\cdot) \in \mathcal{C}^{1}\left(\mathcal{A}_{\mathcal{G}_{\varepsilon, \mu}^{\mathrm{c}}}\right)$. Next, there exists $C>0$ so that, for all $f \in \mathcal{C}_{c}\left(\mathcal{V}^{\mathrm{c}}\right)$,

$$
\begin{aligned}
\left\|\langle\Lambda\rangle^{\epsilon} \otimes\left[V^{\mathrm{c}}(\cdot), \mathrm{i} \mathcal{A}_{\mathcal{G}_{\varepsilon, \mu}^{\mathrm{c}}}\right] \circ f\right\| \leq & \frac{e^{-1 / 2}}{2}\left\|\left(\langle Q+1 / 2\rangle^{\epsilon}(Q+1 / 2)\left[V^{\mathrm{c}}, U^{*}\right]\right) \otimes P^{\mathrm{le}} f\right\| \\
& +\frac{e^{1 / 2}}{2}\left\|\left(\langle Q+1 / 2\rangle^{\epsilon}(Q+1 / 2)\left[V^{\mathrm{c}}, U\right]\right) \otimes P^{\mathrm{le}} f\right\| \\
\leq & \frac{e^{-1 / 2}}{2}\left\|\left(\langle\Lambda\rangle^{\epsilon+1}\left[V^{\mathrm{c}}, U^{*}\right]\right) \otimes P^{\mathrm{le}} f\right\| \\
& +\frac{e^{1 / 2}}{2}\left\|\left(\langle\Lambda\rangle^{\epsilon+1}\left[V^{\mathrm{c}}, U\right]\right) \otimes P^{\mathrm{le}} f\right\| \leq C\|f\|, \quad \text { by }(\mathrm{H} 1) .
\end{aligned}
$$

Finally, the result follow by applying [AmBoGe, Proposition 7.5.7] where the hypotheses are verified in Proposition 4.12.

Here is the most technical part:

Proposition 4.14. Assuming (H2) and (H3), we have $\Delta_{\mathcal{G}_{\varepsilon, \mu}^{\mathrm{c}}} \in \mathcal{C}^{1}\left(\mathcal{A}_{\mathcal{G}_{\varepsilon, \mu}^{\mathrm{c}}}\right)$. More$\operatorname{over}\left[\Delta_{\mathcal{G}_{\varepsilon, \mu}^{\mathrm{c}}}, \mathcal{A}_{\mathcal{G}_{\varepsilon, \mu}^{\mathrm{c}}}\right]_{\circ} \in \mathcal{C}^{0,1}\left(\mathcal{A}_{\mathcal{G}_{\varepsilon, \mu}^{\mathrm{c}}}\right)$. In particular, $\Delta_{\mathcal{G}_{\varepsilon, \mu}^{\mathrm{c}}} \in \mathcal{C}^{1,1}\left(\mathcal{A}_{\mathcal{G}_{\varepsilon, \mu}^{\mathrm{c}}}\right)$.

Proof. We work in $\ell^{2}\left(\mathcal{V}^{\mathrm{c}}, m^{c}\right)$. We first prove that $\widetilde{\Delta}_{\mathcal{G}_{\varepsilon, \mu}^{\mathrm{c}}} \in \mathcal{C}^{1}\left(\mathcal{A}_{\mathcal{G}^{c}}\right)$. By the computation below (with $\epsilon=0$ ), we obtain that there is $c>0$ such that

$$
\left\|\left[\widetilde{\Delta}_{\mathcal{G}_{\varepsilon, \mu}^{\mathrm{c}}, \mu}, \mathcal{A}_{\mathcal{G}^{c}}\right] f\right\|_{\ell^{2}\left(\mathcal{V}^{\mathrm{c}}, m^{\mathrm{c}}\right)} \leq c\|f\|_{\ell^{2}\left(\mathcal{V}^{\mathrm{c}}, m^{\mathrm{c}}\right)}, \quad \forall f \in \mathcal{C}_{c}\left(\mathcal{V}^{\mathrm{c}}\right)
$$

Using Lemma 3.16 and $\left[\mathrm{AmBoGe}\right.$, Theorem 6.3.4], this implies that $\widetilde{\Delta}_{\mathcal{G}_{\varepsilon, \mu}^{\mathrm{c}}} \in \mathcal{C}^{1}\left(\mathcal{A}_{\mathcal{G}^{\mathrm{c}}}\right)$. 
We turn to the $\mathcal{C}^{0,1}$ property. We assume that $(H 2)$ and $(H 3)$ are true then

$$
\begin{aligned}
& \left\|\left\langle\Lambda^{\mathrm{c}}\right\rangle^{\epsilon}\left[\widetilde{\Delta}_{\mathcal{G}_{\varepsilon, \mu}^{\mathrm{c}}, \mu}, \mathcal{A}_{\mathcal{G}^{\mathrm{c}}}\right]_{\circ} f\right\|_{\ell^{2}\left(\mathcal{V}^{\mathrm{c}}, m^{\mathrm{c}}\right)} \leq \sum_{x \in \mathcal{V}^{\mathrm{c}}} m^{\mathrm{c}}(x) \mid \frac{\mathrm{i}}{2}\left(e^{1 / 2}\langle Q-1 / 2\rangle^{1+\epsilon} \otimes P^{\mathrm{le}}\right. \\
& \times\left(\frac{1}{m^{\mathrm{c}}(x)} \sum_{z \sim x} \mathcal{E}^{\mathrm{c}}(x, z) \frac{1+\varepsilon^{\mathrm{c}}(x, z)}{\sqrt{\left(1+\mu^{\mathrm{c}}(x)\right)\left(1+\mu^{\mathrm{c}}(z)\right)}}\right. \\
& \left.-\frac{1}{m^{\mathrm{c}}\left(x_{1}-1, x_{2}\right)} \sum_{z \sim x} \mathcal{E}^{\mathrm{c}}\left(\left(x_{1}-1, x_{2}\right), z\right) \frac{1+\varepsilon^{\mathrm{c}}\left(\left(x_{1}-1, x_{2}\right), z\right)}{\sqrt{\left(1+\mu^{\mathrm{c}}\left(x_{1}-1, x_{2}\right)\left(1+\mu^{\mathrm{c}}(z)\right)\right.}}\right) \\
& \times\left. f\left(x_{1}-1, x_{2}\right)\right|^{2} \\
& +\sum_{x \in \mathcal{V}^{c}} m^{\mathrm{c}}(x) \mid \frac{\mathrm{i}}{2}\left(e^{1 / 2}\langle Q-1 / 2\rangle^{1+\epsilon} \otimes P^{\mathrm{le}}\right) \\
& \times\left(\frac{1}{m^{\mathrm{c}}\left(x_{1}-1, x_{2}\right)} \sum_{z \sim x} \mathcal{E}^{\mathrm{c}}\left(\left(x_{1}-1, x_{2}\right), z\right) \frac{1+\varepsilon^{\mathrm{c}}\left(\left(x_{1}-1, x_{2}\right), z\right)}{\sqrt{\left(1+\mu^{\mathrm{c}}\left(x_{1}-1, x_{2}\right)\left(1+\mu^{\mathrm{c}}(z)\right)\right.}} f(z)\right. \\
& \left.-\frac{1}{m^{\mathrm{c}}(x)} \sum_{z \sim x} \mathcal{E}^{\mathrm{c}}(x, z) \frac{1+\varepsilon^{\mathrm{c}}(x, z)}{\sqrt{\left(1+\mu^{\mathrm{c}}(x)\right)\left(1+\mu^{\mathrm{c}}(z)\right)}} f\left(z_{1}-1, z_{2}\right)\right)\left.\right|^{2} \\
& +\sum_{x \in \mathcal{V}^{\mathrm{c}}} m^{\mathrm{c}}(x) \mid \frac{\mathrm{i}}{2}\left(e^{-1 / 2}\langle Q+1 / 2\rangle^{1+\epsilon} \otimes P^{\mathrm{le}}\right) \\
& \times\left(\frac{1}{m^{\mathrm{c}}\left(x_{1}+1, x_{2}\right)} \sum_{z \sim x} \mathcal{E}^{\mathrm{c}}\left(\left(x_{1}+1, x_{2}\right), z\right) \frac{1+\varepsilon^{\mathrm{c}}\left(\left(x_{1}+1, x_{2}\right), z\right)}{\sqrt{\left(1+\mu^{\mathrm{c}}\left(x_{1}+1, x_{2}\right)\left(1+\mu^{\mathrm{c}}(z)\right)\right.}}\right. \\
& \left.-\frac{1}{m^{\mathrm{c}}(x)} \sum_{z \sim x} \mathcal{E}^{\mathrm{c}}(x, z) \frac{1+\varepsilon^{\mathrm{c}}(x, z)}{\sqrt{\left(1+\mu^{\mathrm{c}}(x)\right)\left(1+\mu^{\mathrm{c}}(z)\right)}}\right)\left.f\left(x_{1}+1, x_{2}\right)\right|^{2} \\
& +\sum_{x \in \mathcal{V}^{\mathrm{c}}} m^{\mathrm{c}}(x) \mid \frac{\mathrm{i}}{2}\left(e^{-1 / 2}\langle Q+1 / 2\rangle^{1+\epsilon} \otimes P^{\mathrm{le}}\right) \\
& \times\left(\frac{1}{m^{\mathrm{c}}(x)} \sum_{z \sim x} \mathcal{E}^{\mathrm{c}}(x, z) \frac{1+\varepsilon^{\mathrm{c}}(x, z)}{\sqrt{\left(1+\mu^{\mathrm{c}}(x)\right)\left(1+\mu^{\mathrm{c}}(z)\right)}} f\left(z_{1}+1, z_{2}\right)\right. \\
& \left.-\frac{1}{m^{\mathrm{c}}\left(x_{1}+1, x_{2}\right)} \sum_{z \sim x} \mathcal{E}^{\mathrm{c}}\left(\left(x_{1}+1, x_{2}\right), z\right) \frac{1+\varepsilon^{\mathrm{c}}\left(\left(x_{1}+1, x_{2}\right), z\right)}{\sqrt{\left(1+\mu^{\mathrm{c}}\left(x_{1}+1, x_{2}\right)\left(1+\mu^{\mathrm{c}}(z)\right)\right.}} f\left(z_{1}, z_{2}\right)\right)\left.\right|^{2} \\
& +\left\|\left\langle\Lambda^{\mathrm{c}}\right\rangle^{\epsilon}\left[W^{\mathrm{c}}, \mathcal{A}_{\mathcal{G}^{\mathrm{c}}}\right]_{\circ} f\right\|_{\ell^{2}\left(\mathcal{V}^{\mathrm{c}}, m^{\mathrm{c}}\right)} \text {. }
\end{aligned}
$$

We treat the first term of $\left\|\left\langle\Lambda^{\mathrm{c}}\right\rangle^{\epsilon}\left[\widetilde{\Delta}_{\mathcal{G}_{\mathcal{E}, \mu}^{\mathrm{c}}}, \mathcal{A}_{\mathcal{G}^{\mathrm{c}}}\right]_{\circ} f\right\|_{\ell^{2}\left(\mathcal{V}^{\mathrm{c}}, m^{\mathrm{c}}\right)}$ in (4.13)

$$
\begin{aligned}
\sum_{x \in \mathcal{V}^{\mathrm{c}}} m^{\mathrm{c}}(x) \mid \frac{\mathrm{i}}{2}\left(e^{1 / 2}\langle Q-1 / 2\rangle^{1+\epsilon} \otimes P^{\mathrm{le}}\right) \\
\quad \times \sum_{z \sim x}\left(\frac{\mathcal{E}^{\mathrm{c}}(x, z)\left(1+\varepsilon^{\mathrm{c}}(x, z)\right)}{m^{\mathrm{c}}(x) \sqrt{\left(1+\mu^{\mathrm{c}}(x)\right)\left(1+\mu^{\mathrm{c}}(z)\right)}}\right. \\
\left.\quad-\frac{\mathcal{E}^{\mathrm{c}}\left(\left(x_{1}-1, x_{2}\right), z\right)\left(1+\varepsilon^{\mathrm{c}}\left(\left(x_{1}-1, x_{2}\right), z\right)\right)}{m^{\mathrm{c}}\left(x_{1}-1, x_{2}\right) \sqrt{\left(1+\mu^{\mathrm{c}}\left(x_{1}-1, x_{2}\right)\left(1+\mu^{\mathrm{c}}(z)\right)\right.}}\right)\left.f\left(x_{1}-1, x_{2}\right)\right|^{2} \\
\leq 2 \sum_{x \in \mathcal{V}^{\mathrm{c}}} m^{\mathrm{c}}(x) \mid \frac{\mathrm{i}}{2}\left(e^{1 / 2}\langle Q-1 / 2\rangle^{1+\epsilon} \otimes P^{\mathrm{le}}\right) \\
\quad \times \sum_{z_{1} \sim x_{1}} \delta_{z_{2}=x_{2}}\left(\frac{\mathcal{E}_{1}^{\mathrm{c}}\left(x_{1}, z_{1}\right)\left(1+\varepsilon^{\mathrm{c}}(x, z)\right)}{m(x) \sqrt{\left(1+\mu^{\mathrm{c}}(x)\right)\left(1+\mu^{\mathrm{c}}(z)\right)}}\right. \\
\left.\quad-\frac{\mathcal{E}_{1}^{\mathrm{c}}\left(x_{1}-1, z_{1}\right)\left(1+\varepsilon^{\mathrm{c}}\left(\left(x_{1}-1, x_{2}\right), z\right)\right)}{m^{\mathrm{c}}\left(x_{1}-1, x_{2}\right) \sqrt{\left(1+\mu^{\mathrm{c}}\left(x_{1}-1, x_{2}\right)\left(1+\mu^{\mathrm{c}}(z)\right)\right.}}\right)\left.f\left(x_{1}-1, x_{2}\right)\right|^{2}
\end{aligned}
$$




$$
\begin{aligned}
& +2 \sum_{x \in \mathcal{V}^{\mathrm{c}}} m^{\mathrm{c}}(x) \mid \frac{\mathrm{i}}{2}\left(e^{1 / 2}\langle Q-1 / 2\rangle^{1+\epsilon} \otimes P^{\mathrm{le}}\right) \\
& \quad \times \sum_{z_{2} \sim x_{2}} \delta_{z_{1}=x_{1}}\left(\frac{\mathcal{E}_{2}\left(x_{2}, z_{2}\right)\left(1+\varepsilon^{\mathrm{c}}(x, z)\right)}{m(x) \sqrt{\left(1+\mu^{\mathrm{c}}(x)\right)\left(1+\mu^{\mathrm{c}}(z)\right)}}\right. \\
& \left.\quad-\frac{\mathcal{E}_{2}\left(x_{2}, z_{2}\right)\left(1+\varepsilon^{\mathrm{c}}\left(\left(x_{1}-1, x_{2}\right), z\right)\right)}{m_{1}^{\mathrm{c}}\left(x_{1}-1\right) m_{2} \sqrt{\left(1+\mu^{\mathrm{c}}\left(x_{1}-1, x_{2}\right)\left(1+\mu^{\mathrm{c}}(z)\right)\right.}}\right)\left.f\left(x_{1}-1, x_{2}\right)\right|^{2} .
\end{aligned}
$$

We focus on (4.14).

$$
\begin{aligned}
& \times \frac{1}{m_{2}}\left(\frac{\sqrt{1+\mu^{\mathrm{c}}\left(x_{1}-1, x_{2}\right)}\left(1+\varepsilon^{\mathrm{c}}\left(x,\left(x_{1}+1, x_{2}\right)\right)\right.}{\sqrt{\left(1+\mu^{\mathrm{c}}(x)\right)\left(1+\mu^{\mathrm{c}}\left(x_{1}+1, x_{2}\right)\right)\left(1+\mu^{\mathrm{c}}\left(x_{1}-1, x_{2}\right)\right)}}\right. \\
& \left.\quad-\frac{\sqrt{1+\mu^{\mathrm{c}}\left(x_{1}+1, x_{2}\right)}\left(1+\varepsilon^{\mathrm{c}}\left(\left(x_{1}-1, x_{2}\right),\left(x_{1}, x_{2}\right)\right)\right)}{\sqrt{\left(1+\mu^{\mathrm{c}}\left(x_{1}-1, x_{2}\right)\left(1+\mu^{\mathrm{c}}(x)\right)\left(1+\mu^{\mathrm{c}}\left(x_{1}+1, x_{2}\right)\right)\right.}}\right)\left.f\left(x_{1}-1, x_{2}\right)\right|^{2} \\
& +4 \sum_{x \in \mathcal{V}^{\mathrm{c}}} m^{\mathrm{c}}(x) \mid \frac{\mathrm{i}}{2}\left(\langle Q-1 / 2\rangle^{1+\epsilon} \otimes P^{\mathrm{le}}\right) \\
& \times \frac{1}{m_{2}}\left(\frac{\sqrt{1+\mu^{\mathrm{c}}\left(x_{1}-2, x_{2}\right)}\left(1+\varepsilon^{\mathrm{c}}\left(x,\left(x_{1}-1, x_{2}\right)\right)\right)}{\sqrt{\left(1+\mu^{\mathrm{c}}(x)\right)\left(1+\mu^{\mathrm{c}}\left(x_{1}-1, x_{2}\right)\right)\left(1+\mu^{\mathrm{c}}\left(x_{1}-2, x_{2}\right)\right)}}\right. \\
& \left.\quad-\frac{\sqrt{1+\mu^{\mathrm{c}}\left(x_{1}-1, x_{2}\right)}\left(1+\varepsilon^{\mathrm{c}}\left(\left(x_{1}-1, x_{2}\right),\left(x_{1}-2, x_{2}\right)\right)\right)}{\sqrt{\left(1+\mu^{\mathrm{c}}\left(x_{1}-1, x_{2}\right)\left(1+\mu^{\mathrm{c}}\left(x_{1}-2, x_{2}\right)\right)\left(1+\mu^{\mathrm{c}}\left(x_{1}-1, x_{2}\right)\right)\right.}}\right) \\
& \quad \times\left. f\left(x_{1}-1, x_{2}\right)\right|^{2} .
\end{aligned}
$$

Now, we concentrate on (4.16). (4.17) can be done in the same way. Since the assertions $(H 2)$ and $(H 3)$ hold true then there exists an integer $c$, such that

$$
\begin{aligned}
& 4 \sum_{x \in \mathcal{V}^{\mathrm{c}}} m^{\mathrm{c}}(x) \mid \frac{\mathrm{i}}{2}\left(e(Q-1 / 2)^{1+\epsilon} \otimes P^{\mathrm{le}}\right) \\
& \quad \times \frac{1}{m_{2}}\left(\frac{\sqrt{1+\mu^{\mathrm{c}}\left(x_{1}-1, x_{2}\right)}\left(1+\varepsilon^{\mathrm{c}}\left(x,\left(x_{1}+1, x_{2}\right)\right)\right.}{\sqrt{\left(1+\mu^{\mathrm{c}}(x)\right)\left(1+\mu^{\mathrm{c}}\left(x_{1}+1, x_{2}\right)\right)\left(1+\mu^{\mathrm{c}}\left(x_{1}-1, x_{2}\right)\right)}}\right. \\
& \left.\quad-\frac{\sqrt{1+\mu^{\mathrm{c}}\left(x_{1}+1, x_{2}\right)}\left(1+\varepsilon^{\mathrm{c}}\left(\left(x_{1}-1, x_{2}\right),\left(x_{1}, x_{2}\right)\right)\right)}{\sqrt{\left(1+\mu^{\mathrm{c}}\left(x_{1}-1, x_{2}\right)\left(1+\mu^{\mathrm{c}}(x)\right)\left(1+\mu^{\mathrm{c}}\left(x_{1}+1, x_{2}\right)\right)\right.}}\right)\left.f\left(x_{1}-1, x_{2}\right)\right|^{2} \\
& =4 \sum_{x \in \mathcal{V}^{\mathrm{c}}} m^{\mathrm{c}}(x) \mid \frac{\mathrm{i}}{2}\left(e\langle Q-1 / 2\rangle^{1+\epsilon} \otimes P^{\mathrm{le}}\right) \frac{1}{m_{2}} \\
& \quad \times\left(\frac{\left(\mu^{\mathrm{c}}\left(x_{1}-1, x_{2}\right)-\mu^{\mathrm{c}}\left(x_{1}+1, x_{2}\right)\right) \varepsilon^{\mathrm{c}}\left(x,\left(x_{1}+1, x_{2}\right)\right)}{\left(\sqrt{1+\mu^{\mathrm{c}}\left(x_{1}-1, x_{2}\right)}+\sqrt{1+\mu^{\mathrm{c}}\left(x_{1}+1, x_{2}\right)}\right)}\right) \\
& \left.\quad \times \frac{1}{\sqrt{\left(1+\mu^{\mathrm{c}}(x)\right)\left(1+\mu^{\mathrm{c}}\left(x_{1}+1, x_{2}\right)\right)\left(1+\mu^{\mathrm{c}}\left(x_{1}-1, x_{2}\right)\right)}}\right)
\end{aligned}
$$




$$
\begin{aligned}
& +4 \sum_{x \in \mathcal{V}^{\mathrm{c}}} m^{\mathrm{c}}(x) \mid \frac{\mathrm{i}}{2}\left(e\langle Q-1 / 2\rangle^{1+\epsilon} \otimes P^{\mathrm{le}}\right) \frac{1}{m_{2}} \\
& \quad \times\left(\frac{\sqrt{1+\mu^{\mathrm{c}}\left(x_{1}+1, x_{2}\right)}\left(\varepsilon^{\mathrm{c}}\left(x,\left(x_{1}+1, x_{2}\right)\right)-\varepsilon^{\mathrm{c}}\left(\left(x_{1}-1, x_{2}\right), x\right)\right.}{\sqrt{\left(1+\mu^{\mathrm{c}}(x)\right)\left(1+\mu^{\mathrm{c}}\left(x_{1}+1, x_{2}\right)\right)\left(1+\mu^{\mathrm{c}}\left(x_{1}-1, x_{2}\right)\right)}}\right. \\
& \quad+\frac{\mu^{\mathrm{c}}\left(x_{1}-1, x_{2}\right)-\mu^{\mathrm{c}}\left(x_{1}+1, x_{2}\right)}{\left(\sqrt{1+\mu^{\mathrm{c}}\left(x_{1}-1, x_{2}\right)}+\sqrt{1+\mu^{\mathrm{c}}\left(x_{1}+1, x_{2}\right)}\right)} \\
& \left.\quad \times \frac{1}{\sqrt{\left(1+\mu^{\mathrm{c}}(x)\right)\left(1+\mu^{\mathrm{c}}\left(x_{1}+1, x_{2}\right)\right)\left(1+\mu^{\mathrm{c}}\left(x_{1}-1, x_{2}\right)\right)}}\right)\left.f\left(x_{1}-1, x_{2}\right)\right|^{2} \\
& \leq c\|f\|_{\ell^{2}\left(\mathcal{V}^{\mathrm{c}}, m^{\mathrm{c}}\right)}^{2},
\end{aligned}
$$

and in the same way, we deal with (4.15) and $\left\|\left\langle\Lambda^{\mathrm{c}}\right\rangle^{\epsilon}\left[W^{\mathrm{c}}, \mathcal{A}_{\mathcal{G}^{\mathrm{c}}}\right]_{\circ} f\right\|_{\ell^{2}\left(\mathcal{V}^{\mathrm{c}}, m^{\mathrm{c}}\right)}$. By density, we have proven that there exists $c>0$ such that

$$
\left\|\left\langle\Lambda^{\mathrm{c}}\right\rangle^{\epsilon}\left[\widetilde{\Delta}_{\mathcal{G}_{\varepsilon, \mu}^{\mathrm{c}}}, \mathcal{A}_{\mathcal{G}^{\mathrm{c}}}\right]_{\circ} f\right\|_{\ell^{2}\left(\mathcal{V}^{\mathrm{c}}, m^{\mathrm{c}}\right)}^{2} \leq c\|f\|_{\ell^{2}\left(\mathcal{V}^{\mathrm{c}}, m^{\mathrm{c}}\right)}^{2} .
$$

Finally, by applying [AmBoGe, Proposition 7.5.7] with $\mathcal{G}:=\mathcal{D}\left(\Delta_{\mathcal{G}_{\varepsilon, \mu}^{\mathrm{c}}}\right)$ where the hypotheses are verified in Proposition 4.6, we find the result.

\section{REFERENCES}

[AmBoGe] W.O. Amrein, A. Boutet de Monvel, and V. Georgescu, $C_{0}$-groups, commutator methods and spectral theory of N-body hamiltonians, Birkhäuser (1996).

[AlFr] C. Allard and R. Froese: A Mourre estimate for a Schrödinger operator on a binary tree, Rev. Math. Phys. 12 (2000), no. 12, 1655-1667.

[BaFrSi] V. Bach, J. Fröhlich, and I.M. Sigal: Quantum electrodynamics of confined nonrelativistic particles, Adv. in Math. 137 (1998), 299-395.

[BoGo] M. Bonnefont and S. Golénia: Essential spectrum and Weyl asymptotics for discrete Laplacians, Ann. Fac. Sci. Toulouse Math. (6) 24 (2015), no. 3, 563-624.

[BoKeGoLiMü] M. Bonnefont, M. Keller, S. Golénia, S.Liu, and F. Münch: Magnetic sparseness and Schrödinger operators on graphs, arXiv:1711.10418v1 [math.SP].

[BoCaHäMi] J.F. Bony, R. Carles, D. Häffner, and L. Michel: Scattering theory for the Schrödinger equation with repulsive potential, J. Math. Pures Appl. 84 (2005), no. 5, 509-579.

[BoGo] N. Boussaid and S. Golénia: Limiting absorption principle for some long range pertubations of Dirac systems at threshold energies, Comm. Math. Phys. 299 (2010), 677-708.

[BoSa] A. Boutet de Monvel and J. Sahbani: On the spectral properties of discrete Schrödinger operators: the multi-dimensional case, Rev. Math. Phys. 11 (1999), no. 9, 1061-1078.

[BrKe] J. Breuer and M. Keller: Spectral Analysis of Certain Spherically Homogeneous Graphs, Oper. Matrices 7 (2013), no. 4, 825-847.

[CaGrHu] L. Cattaneo, G. M. Graf, and W. Hunziker: A general resonance theory based on Mourre's inequality, Ann. H. Poincaré. 7 (2006), no. 3, 583-601.

[Ch] F.R.K. Chung: Spectral graph theory, CBMS Regional Conference Series in Mathematics, 92. Published for the Conference Board of the Mathematical Sciences, Washington, DC; by the American Mathematical Society, Providence, RI, 1997. xii+207 pp. ISBN: 0-8218-0315-8.

[CoToTr] Y. Colin De Verdière, N. Torki-Hamza and F. Truc: Essential self-adjointness for combinatorial Schrödinger operators II- Metrically non complete graphs, Mathematical Physics Analysis and Geometry 14, 1 (2011) 21-38.

[DeJa] J. Dereziński and V. Jakšić: Spectral theory of Pauli-Fierz operators, J. Funct. Anal. 180 (2001), no. 2, 243-327.

[FrHe] R.G. Froese and I. Herbst: Exponential bounds and absence of positive eigenvalues for N-body Schrödinger operators, Comm. Math. Phys. 87 (1982), 429-447.

[GeGé] V. Georgescu and C. Gérard: On the Virial Theorem in Quantum Mechanics, Commun. Math. Phys. 208, No.2, 275-281 (1999).

[GeGéMø] V. Georgescu, C. Gérard, and J.S. Møller: Commutators, $C_{0}$-semigroups and resolvent estimates, J. Funct. Anal. 216 (2004), no. 2, 303-361.

[GeGo] V. Georgescu and S. Golénia: Isometries, Fock spaces, and spectral analysis of Schrödinger operators on trees. J. Funct. Anal. 227 (2005), no. 2, 389-429. 
[Gé] C. Gérard: A proof of the abstract limiting absorption principle by energy estimates, J. Funct. Anal. 254 (2008), no. 11, 2707-2724.

[Go] S. Golénia: Hardy inequality and asymptotic eigenvalue distribution for discrete Laplacians, J. Funct. Anal. 266 (2014), no. 5, 2662-2688.

[GoJe1] S. Golénia, T. Jecko: A new look at Mourre's commutator theory, Compl. Anal. Oper. Theory, Vol. 1, No. 3, p. 399-422, (2007).

[GoJe2] S. Golénia and T. Jecko: Weighted Mourre's commutator theory, application to Schrödinger operators with oscillating potential. J. of Operator Theory. $\mathbf{7 0}$ (2012).

[GoTr] S. Golénia and F. Truc: The magnetic Laplacian acting on discrete cusp, Doc. Math. 22 (2017), 1709-1727.

[GoMo] S. Golénia and S. Moroianu: Spectral analysis of magnetic Laplacians on conformally cusp manifolds, Ann. Henri Poincaré. 9 (2008), no. 1, 131-179.

[HaKe] S. Haeseler and M. Keller: Generalized solutions and spectrum for Dirichlet forms on graphs, Boundaries and Spectral Theory, Progress in Probability, 2011 Birkhäuser, 181-201.

[Ha] F. Harary: Graph Theory, Addison-Wesley Publishing Co. Reading, Mass-Menlo Park, Calif-London 1969 ix+274 pp.

[HiNo] Y. Higuchi and Y. Nomura: Spectral stucture of the Laplacian on a covering graph, European Journal of Combinatorics, 30 (2009), 570-585.

[HiSh] Y. Higuchi and T. Shirai: Weak Bloch property for discrete magnetic Schrödinger operators, Nagoya Math. J. 161 (2001), 127-154.

[HuSi] W. Hunziker and I.M. Sigal: The quantum N-body problem, J. Math. Phys. 41 (2000), no. 6, 3448-3510.

[JeMoPe] A. Jensen, E. Mourre, and P. Perry: Multiple commutator estimates and resolvent smoothness in quantum scattering theory, Ann. Inst. H. Poincaré. vol. 41 (1984), no. 2, 207-225.

[MăRiTi] M. Măntoiu, S. Richard, and R. Tiedra de Aldecoa: Spectral analysis for adjacency operators on graphs, Ann. Henri Poincaré 8 (2007), no. 7, 1401-1423.

[Mic] N. Michaelis: Spectral theory of anisotropic discrete Schrödinger operators in dimension one, master thesis available at https://www.math.ubordeaux.fr/ sgolenia/Fichiers/diplom.michaelis.pdf.

[MoTr] A. Morame and F. Truc: Magnetic bottles on geometrically finite hyperbolic surfaces, J. Geom. Phys. 59 (2009), no. 7, 1079-1085.

[Mo1] E. Mourre: Absence of singular continuous spectrum for certain self-adjoint operators. Comm. Math. Phys. 78 (1980), 391-408.

[Mo2] E. Mourre: Opérateurs conjugués et propriétés de propagation, Comm. Math. Phys. 91 (1983), 279-300.

[PaRi] D. Parra and S. Richard: Spectral and scattering theory for Schrödinger operators on perturbed topological crystals, Rev. Math. Phys. 30 (2018), no. 4, 1850009, 39 pp.

[Pu] C.R. Putnam: Commutator properties of Hilbert space operators and related topics, Springer Verlag (1967).

[ReSi] M. Reed and B. Simon: Methods of Modern Mathematical Physics, Tome I-IV: Analysis of operators Academic Press.

[RoTa] D. Robert and H. Tamura: Semiclassical estimates for resolvents and asymptotics for total cross-section, Ann. Inst. H. Poincaré. 46 (1987), 415-442.

[Roy] J. Royer: Limiting absorption principle for the dissipative Helmholtz equation, Comm. Partial Differntial Equations. 35 (2010), 1458-1489.

[Sa] J. Sahbani: Mourre's theory for some unbounded Jacobi matrices, J. Approx. Theory. 135 (2005), no. 2, 233-244.

[Th] W.P. Thurston: Three-Dimensional Geometry and Topology - Volume 1, Edited by Silvio Levy. Princeton Mathematical Series. 35. Princeton University Press, Princeton, NJ, (1997).

[Wa] X.P. Wang: Semiclassical resolvent estimates for $N$-body Schrödinger operators, J. Funct. Anal. 97 (1991), 466-483.

[WaZh] X.P. Wang and P. Zhang: High frequency limit of the Helmholtz equation with variable refraction index, J. Funct. Anal. 230 (2006), 116-168.

[We] J. Weidmann: Zur Spektraltheorie von Sturm-Liouville-Operatoren, Math. Z. 98 (1962), 268-302. 
Université de Gafsa, Campus Universitaire 2112, Tunisie

E-mail address: athmouninassim@yahoo.fr

Université de Sfax. Route de la Soukra KM 4 - B.P. N 802 - 3038 Sfax

E-mail address: ennaceur.marwa27@gmail.com

Univ. Bordeaux, Bordeaux INP, CNRS, IMB, UMR 5251, F-33400 Talence, France

E-mail address: sylvain.golenia@math.u-bordeaux.fr 